\title{
Non-additive geometry
}

\author{
M. J. Shai Haran
}

\begin{abstract}
We develop a language that makes the analogy between geometry and arithmetic more transparent. In this language there exists a base field $\mathbb{F}$, 'the field with one element'; there is a fully faithful functor from commutative rings to $\mathbb{F}$-rings; there is the notion of the $\mathbb{F}$-ring of integers of a real or complex prime of a number field $K$ analogous to the $p$-adic integers, and there is a compactification of Spec $O_{K}$; there is a notion of tensor product of $\mathbb{F}$-rings giving the product of $\mathbb{F}$-schemes; in particular there is the arithmetical surface $\operatorname{Spec} O_{K} \times \operatorname{Spec} O_{K}$, the product taken over $\mathbb{F}$.
\end{abstract}

\section{Contents}

Introduction

$1 \mathbb{F}$, the field with one element $\quad 621$

1.1 The category $\mathbb{F} \ldots \ldots \ldots \ldots$. . . . . . . . . . . . . . . 622

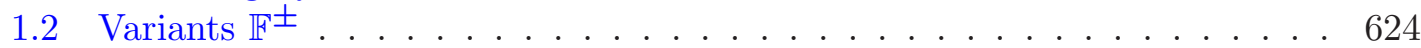

1.3 The 'algebraic closure' $\overline{\mathbb{F}}$ of $\mathbb{F} \ldots \ldots \ldots \ldots . \ldots \ldots 625$

2 F-rings, variants, examples $\quad 627$

2.1 Definition of $\mathbb{F}$-rings . . . . . . . . . . . . . . . . . 627

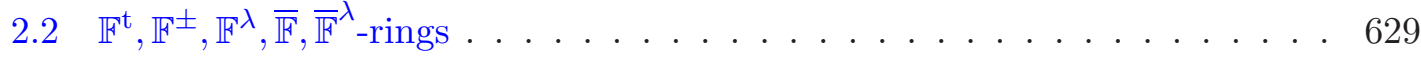

2.3 Examples of $\mathbb{F}$-rings $\ldots \ldots \ldots \ldots . \ldots \ldots . \ldots \ldots 2$

3 Modules $\quad 636$

3.1 Definitions and examples . . . . . . . . . . . . . . . 637

$3.2 \quad A$-submodules and equivalence $A$-modules . . . . . . . . . . . . . . 639

3.3 Operations on submodules . . . . . . . . . . . . . . . . 643

3.4 Operations on modules . . . . . . . . . . . . . . . . . 643

4 Ideals and primes $\quad 650$

$4.1 \quad H$-ideals and primes . . . . . . . . . . . . . . . . 650

$4.2 \quad S$-prime ideals and $H$-E-ideals $\ldots \ldots \ldots \ldots \ldots \ldots$. . . . . . . 654

$\begin{array}{llr}5 & \text { Localization and structural sheaf } \mathcal{O}_{A} & 658\end{array}$

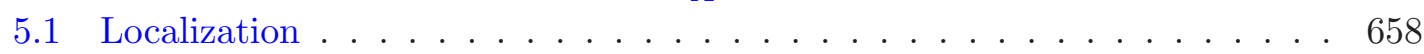

5.2 Structural sheaf $\mathcal{O}_{A} \ldots \ldots \ldots \ldots \ldots 6 . \ldots \ldots \ldots$

6 Schemes $\quad 665$

6.1 Locally $\mathbb{F}$-ring spaces . . . . . . . . . . . . . . . . . . . . 665

6.2 Zariski $\mathbb{F}$-schemes . . . . . . . . . . . . . . . . . . . 667

$6.3 \mathbb{F}$-schemes and the compactified $\overline{\mathrm{Spec} \mathbb{Z}} \ldots \ldots \ldots \ldots 8$

$\begin{array}{lll}7 & \text { Fibred products } & 671\end{array}$

7.1 Fibred sums of $\mathbb{F}$-rings . . . . . . . . . . . . . . . . . 671

7.2 Fibred product of $\mathbb{F}$-schemes, the case of $\overline{\operatorname{Spec} \mathbb{Z}} \times \overline{\text { Spec } \mathbb{Z}} \ldots \ldots$. . . . . 674

Received 28 June 2006, accepted in final form 13 September 2006.

2000 Mathematics Subject Classification 11G99.

Keywords: arithmetical surface, compactified Spec $\mathbb{Z}$, Riemann hypothesis, ABC conjecture.

This journal is (C) Foundation Compositio Mathematica 2007. 
8.1 F-monoids . . . . . . . . . . . . . . . . . . 676

8.2 Modules over an F-monoid . . . . . . . . . . . . . . . . . 677

8.3 Functorial operations on modules . . . . . . . . . . . . . . . . 680

References

\section{Introduction}

The ancient idea of making arithmetic into geometry engaged the minds of great mathematicians such as Kummer, Kronecker, Dedekind, Hensel, Hasse, Minkowski, and especially Artin and Weil. It is a beautiful quest inspired by the similarity between the ring of integers $\mathbb{Z}$, and the ring of polynomials $Z=\mathbb{k}[x]$ over a field $\mathbb{k}$; for closer similarity the 'function field' case is relevant where $\mathbb{k}=\mathbb{F}_{q}$ is a finite field. There is induced similarity of the fraction fields, the field of rational numbers $\mathbb{Q}$ and the field of rational functions $Q=\mathbb{k}(x)$. For a prime $p$ of $\mathbb{Z}$, we have the $p$-adic integers

and its field of fractions

$$
\mathbb{Z}_{p}=\lim _{\longleftarrow} \mathbb{Z} / p^{n}
$$

$$
\mathbb{Q}_{p}=\mathbb{Z}_{p}\left[\frac{1}{p}\right]
$$

with dense embeddings $\mathbb{Z} \subseteq \mathbb{Z}_{p}$ and $\mathbb{Q} \subseteq \mathbb{Q}_{p}$. The geometric analogues are the power series ring

$$
Z_{f}=\lim _{\longleftarrow} Z / f^{n}=\mathbb{k}_{f}[[f]]
$$

and the field of Laurent series

$$
Q_{f}=Z_{f}\left[\frac{1}{f}\right]=\mathbb{k}_{f}((f)),
$$

for $f$ a prime of $Z$, where $\mathbb{k}_{f}=\mathbb{k}[x] /(f)$, and the embeddings $Z \subseteq Z_{f}$ (respectively $Q=\mathbb{k}(x) \subseteq$ $Q_{f}$ ) correspond to expanding a polynomial (respectively a rational function) into a power series (respectively a Laurent series) in $f$. Finite extensions of $Q=\mathbb{k}(x)$ correspond one-to-one with the smooth projective curves $Y$ defined over finite extensions of $\mathbb{k}$, and finite extensions of $\mathbb{Q}$ are the number fields. There are two main difficulties with this analogy that we are going to describe, the problem of the real prime of $\mathbb{Q}$, and the problem of the arithmetical surface, that is defining for $\operatorname{Spec}(\mathbb{Z})$ the analogue of the geometric surface $Y \times_{\mathbb{k}} Y$.

From geometry we know that, in order to have theorems, we must pass from affine to projective geometry, in particular we need to add the point at infinity $\infty$ to the affine line, $\mathbb{P}_{\mathbb{k}}^{1}=\mathbb{A}_{\mathbb{k}}^{1} \cup\{\infty\}$. This corresponds to the ring

$$
Z_{\infty}=\lim _{\longleftarrow} \mathbb{k}\left[\frac{1}{x}\right] /\left(\frac{1}{x}\right)^{n}=\mathbb{k}\left[\left[\frac{1}{x}\right]\right],
$$

and its fraction field

$$
Q_{\infty}=Z_{\infty}[x]=\mathbb{k}\left(\left(\frac{1}{x}\right)\right)
$$

the embedding $Q \subseteq Q_{\infty}$ is the expansion of a rational function as a Laurent series in $1 / x$. The analogue of $\infty$ for $\mathbb{Q}$ is the real prime, which we denote by $\eta$. The associated field is $\mathbb{Q}_{\eta}=\mathbb{R}$, the real numbers. But there is no analogue $\mathbb{Z}_{\eta}$ of $Z_{\infty}$. For finite primes $p$,

$$
\mathbb{Z}_{p}=\left\{x \in \mathbb{Q}_{p},|x|_{p} \leqslant 1\right\} .
$$

We have to carry remainder when we add elements of $\mathbb{Z}_{p}-$ unlike the simple addition of power series in $Z_{f}$ or $Z_{\infty}$. We carry the remainder from the larger scale $p^{j}$ to the smaller scale $p^{j+1}$, hence

$$
|x+y|_{p} \leqslant \max \left\{|x|_{p},|y|_{p}\right\},
$$




\section{J. Shai HARAN}

and $\mathbb{Z}_{p}$ is closed under addition. In contrast, when we add real numbers, we carry the remainder from the smaller to the larger scale, we have only the weaker triangle inequality

$$
|x+y|_{\eta} \leqslant|x|_{\eta}+|y|_{\eta}
$$

and $\left\{x \in \mathbb{Q}_{\eta},|x|_{\eta} \leqslant 1\right\}=[-1,1]$ is not closed under addition.

The second problem is that in geometry we have products, in particular the affine plane $\mathbb{A}^{2}=$ $\mathbb{A}^{1} \times \mathbb{A}^{1}$, with the ring of polynomial functions $\mathbb{k}[x] \otimes_{\mathbb{k}} \mathbb{k}[x]=\mathbb{k}\left[x_{1}, x_{2}\right]$, the tensor product (三 sum in the category of $\mathbb{k}$-algebras) of $Z$ with itself. When we try to find the analogous arithmetical surface, we find $\mathbb{Z} \otimes \mathbb{Z}=\mathbb{Z}$. The integers $\mathbb{Z}$ are the initial object in the category of rings, so its tensor product ( $\equiv$ sum in the category of rings) with itself is just $\mathbb{Z}$. For any geometry that is based on rings, Spec $\mathbb{Z}$ will be the final object, and Spec $\mathbb{Z} \times \operatorname{Spec} \mathbb{Z}=$ Spec $\mathbb{Z}$, which means the arithmetical surface reduces to the diagonal!

Motivated by the Weil conjectures, Grothendieck developed the modern language of algebraic geometry, the language of schemes [EGA], based on commutative rings. Grothendieck came from a background of functional analysis, where the paradigm of 'geometry = commutative rings' was first set. It is the famous Gelfand-Naimark theorem on the equivalence of the category of (compact, Hausdorff) topological spaces and the category of commutative (unital) $\mathbb{C}^{*}$-algebras. This equivalence is given by associating with the topological space $X$ the algebra

$$
\mathbb{C}(X)=\{f: X \rightarrow \mathbb{C}, f \text { continuous }\},
$$

using addition and multiplication (and conjugation, and norm) of $\mathbb{C}$ to define the similar structure on $\mathbb{C}(X)$, giving rise to the structure of ring (and $\mathbb{C}^{*}$-algebra structure) on $\mathbb{C}(X)$. The axioms of a commutative $\mathbb{C}^{*}$-algebra are generalizations of the axioms of $\mathbb{C}$ : when $X=\{*\}$ reduces to a point, $\mathbb{C}(*)=\mathbb{C}$. It is clear that there is no connection between addition and multiplication of $\mathbb{C}$ and the geometry of $X$. The language of rings (and commutative $\mathbb{C}^{*}$-algebras) is just one convenient way in which to encode geometry.

With the goal of finding the arithmetical surface, the idea of abandoning addition has recently appeared in the literature. Soulé [Sou04] talks of the 'field with one element' $\mathbb{F}$, and tries to define $\mathbb{F}$-varieties as a subcollection of $\mathbb{Z}$-varieties. Kurokawa, Ochiai and Wakayama [KOW03] were the first to suggest abandoning addition, and working instead with the multiplicative monoids. This idea was further described in Deitmar [Dei05], but note that the spectra of monoids always looks like the spectra of a local ring: the non-invertible elements are the unique maximal ideal. For Kurokawa there is also a 'zeta world' of analytic functions that encode geometry, where the field $\mathbb{F}$ is encoded by the identity function of $\mathbb{C}$; see Manin [Man95].

Here we take our clues from the problem of the real prime to understand $\mathbb{F}$, and then develop the language of geometry based on the concept of $\mathbb{F}$-ring. Denote by $|x|_{\eta}$ the euclidian norm of $x=\left(x_{1}, \ldots, x_{n}\right) \in \mathbb{R}^{n}$, i.e.

$$
|x|_{\eta}=\sqrt{\sum_{i}\left|x_{i}\right|_{\eta}^{2}} .
$$

We have the fundamental Cauchy-Schwartz inequality

$$
|x \circ y|_{\eta}=\left|x_{1} y_{1}+\cdots+x_{n} y_{n}\right|_{\eta} \leqslant|x|_{\eta} \cdot|y|_{\eta} .
$$

Hence $[-1,1]$ will contain $x_{1} y_{1}+\cdots+x_{n} y_{n}=x \circ y$, whenever $|x|_{\eta},|y|_{\eta} \leqslant 1$, although it is not closed under addition. Moreover, unlike addition, matrix multiplication behaves well in the real prime: $|a \circ b|_{\eta} \leqslant|a|_{\eta} \cdot|b|_{\eta}$ for real or complex matrices $a, b$ where $|\cdot|_{\eta}$ is the operator norm. Within matrix multiplication there is encoded addition, but we have to take matrix multiplication as the more fundamental operation. We add also the operations of direct sum and of tensor product of matrices. Our analogue of $\mathbb{Z}_{p}$ (respectively of the localization $\mathbb{Z}_{(p)}$ ) for the real prime $\eta$ is the category $\mathcal{O}_{\mathbb{R}, \eta}$ 


\section{NON-ADDITIVE GEOMETRY}

(respectively $\mathcal{O}_{\mathbb{Q}, \eta}$ ) with objects the finite sets and morphisms from $X$ to $Y$ given by the $Y \times X$ matrices with real (respectively rational) coefficients and with operator norm $\leqslant 1$; these matrices are closed under the operations of direct sums and tensor products (but are not closed under addition).

Remembering that the quantum area in physics started with Heisenberg's discovery of matrix multiplication as the fundamental operation describing the energy levels of microscopic systems, perhaps in the future also physics will benefit from the language of non-additive geometry.

The contribution to arithmetic is evident: the real integers $\mathbb{Z}_{\eta}$ become a real object, and the arithmetical surface exists and does not reduce to the diagonal. Some well-known conjectures of arithmetic (Riemann hypothesis, ABC, ...) are easy theorems in the geometric analogue of a curve $C$ over a finite field. This is because we can form the surface $C \times C$. The knowledge of the first infinitesimal neighborhood of the diagonal $C$ within $C \times C$, i.e. of differentials, is often sufficient to prove theorems in geometry whose arithmetic analogues are deep conjectures. Therefore, the further study of the arithmetical surface $\mathbb{F}(\mathbb{Z}) \otimes_{\mathbb{F}} \mathbb{F}(\mathbb{Z})$, its compactification using $\mathbb{F}(\mathbb{Z}) \otimes_{\mathbb{F}} \mathbb{Z}_{\eta}$, and the arithmetic first infinitesimal neighborhood of the diagonal are important challenges. Here we give only the foundations of the language of non-additive geometry.

In $\S 1$ we decipher what is the 'field with one element' $\mathbb{F}$. The idea is that, while $\mathbb{F}$ degenerates into one (or two) elements, there is a whole category of ' $\mathbb{F}$-valued matrices'. There are various degrees of structures one can impose on $\mathbb{F}$. In $\S 2$ we give the basic notion of an $\mathbb{F}$-ring. As important examples of $\mathbb{F}$-rings we have: $\mathbb{F}(A)$, the $\mathbb{F}$-ring attached to a commutative ring $A ; \mathcal{O}_{\eta}$, the $\mathbb{F}$-ring of 'integers' at a real or complex prime $\eta$ of a number field; and its residue field $\mathbb{F}_{\eta}$, the $\mathbb{F}$-ring of partial isometries. In $\S 3$ we give the elementary theory of modules over $\mathbb{F}$-rings, and discuss (fibred) sums and products, kernels and cokernels, free modules, tensor products, and base change. A novelty of the non-additive setting is the connection between submodules and equivalence modules of a given module.

In $\S 4$ we give the elementary theory of ideals and primes. We associate with any $\mathbb{F}$-ring $A$ its spectrum Spec $A$, a compact sober space with respect to the 'Zariski topology'. (A topological space is sober if every closed irreducible subset has a unique generic point.) In $\S 5$ we give the theory of localization. It gives rise to a sheaf of $\mathbb{F}$-rings over Spec $A$. By gluing such spectra we get Zariski $\mathbb{F}$-schemes. In $\S 6$ we give the theory of $\mathbb{F}$-schemes which are the pro-objects of Zariski $\mathbb{F}$-schemes. As important examples we give the compactification of $\operatorname{Spec} \mathbb{Z}$ and of $\operatorname{Spec} O_{K}, K$ a number field. This is our solution to the problem of the real prime.

In $\S 7$ we give the tensor product, the (fibred) sum in the category of $\mathbb{F}$-rings, and we obtain the (fibred) product in the categories of Zariski $\mathbb{F}$-schemes and of $\mathbb{F}$-schemes. As an important example we define and describe the fibred product $\mathbb{F}(\mathbb{Z}) \otimes_{\mathbb{F}} \mathbb{F}(\mathbb{Z})$, its compactification, and its generalization for number fields. This is our solution to the problem of the arithmetical surface. In $\S 8$ we work over a fixed $\mathbb{F}$-ring $\mathrm{F}$, and repeat the above constructions in the category of monoid objects in F-modules. Everything goes through, the tensor product of F-monoids is just their tensor product as modules, so we avoid the complicated product of $\S 7$, but the functor from commutative rings to $\mathbb{F}$-monoids is not fully faithful.

\section{1. $\mathbb{F}$, the field with one element}

We define a category $\mathbb{F}$ with objects the finite sets endowed with two symmetric monoidal structures $\oplus$ and $\otimes$. The unit element $[0]$ for $\oplus$ is the initial and final object of the category, and $\otimes$ is distributive over $\oplus$. 


\section{J. Shai HARAN}

\subsection{The category $\mathbb{F}$}

We consider $\mathbb{F}$-vector spaces as finite sets $X$ with a distinguished 'zero' element $0_{X} \in X$, and set $X^{+}=X \backslash\left\{0_{X}\right\}$. For a commutative ring $A$, we let

$$
A \cdot X=\bigoplus_{x \in X^{+}} A \cdot x
$$

denote the free $A$-module with basis $X^{+}$, and think about $A \cdot X$ as $A \otimes_{\mathbb{F}} X$ obtained by base extension from $\mathbb{F}$ to $A$. We let

$$
\mathbb{F}[A]_{Y, X}=\operatorname{Hom}_{A}(A \cdot X, A \cdot Y),
$$

the $Y^{+} \times X^{+}$matrices with values in $A$. The base extension of $X$ from $\mathbb{F}$ to $\mathbb{Z}_{\eta}$ and to $\mathbb{Q}_{\eta}=\mathbb{R}$ gives $\mathbb{Z}_{\eta} \cdot X$ and $\mathbb{Q}_{\eta} \cdot X: \mathbb{Q}_{\eta} \cdot X$ is the real vector space with basis $X^{+}$, and $\mathbb{Z}_{\eta} \cdot X$ is the subset of $\mathbb{Q}_{\eta} \cdot X$ of vectors with norm $\leqslant 1$ in the inner product given by decreeing $X^{+}$to be an orthonormal basis. We have

$$
\mathbb{F}\left[\mathbb{Q}_{\eta}\right]_{Y, X}=\operatorname{Hom}_{\mathbb{Q}_{\eta}}\left(\mathbb{Q}_{\eta} \cdot X, \mathbb{Q}_{\eta} \cdot Y\right),
$$

the $Y^{+} \times X^{+}$real-valued matrices, and

$$
\left(\mathbb{Z}_{\eta}\right)_{Y, X}=\left\{f \in \mathbb{F}\left[\mathbb{Q}_{\eta}\right]_{Y, X}, f\left(\mathbb{Z}_{\eta} \cdot X\right) \subseteq \mathbb{Z}_{\eta} \cdot Y\right\}=\left\{f,|f|_{\eta} \leqslant 1\right\}
$$

where $|f|_{\eta}$ denotes the operator norm on $\mathbb{F}\left[\mathbb{Q}_{\eta}\right]_{Y, X}$.

A map of finite sets $\varphi: X \rightarrow Y$, preserving the zero elements $\varphi\left(0_{X}\right)=0_{Y}$, induces an $A$-linear map

$$
\varphi_{A}: A \cdot X \rightarrow A \cdot Y, \quad \varphi_{A} \in \mathbb{F}[A]_{Y, X}
$$

For $\varphi_{\mathbb{Q}_{\eta}}: \mathbb{Q}_{\eta} \cdot X \rightarrow \mathbb{Q}_{\eta} \cdot Y$ to map $\mathbb{Z}_{\eta} \cdot X$ into $\mathbb{Z}_{\eta} \cdot Y$ it is necessary and sufficient that $\varphi$ is an injection of $X \backslash \varphi^{-1}\left(0_{Y}\right)$ into $Y$. Thus we set

$$
\mathbb{F}_{Y, X}=\left\{\varphi: X \rightarrow Y, \varphi\left(0_{X}\right)=0_{Y},\left.\varphi\right|_{X \backslash \varphi^{-1}\left(0_{Y}\right)} \text { injective }\right\},
$$

and we view $\mathbb{F}$ as the category with objects finite sets with a distinguished zero element, and with arrows $\mathbb{F}_{Y, X}=\operatorname{Hom}_{\mathbb{F}}(X, Y)$. In practice, we shall ignore the distinguished elements, and view $\mathbb{F}$ as the category with objects finite sets (without a distinguished zero element), and with arrows the partial bijections

$$
\mathbb{F}_{Y, X}^{\prime}=\{\varphi: V \stackrel{\sim}{\rightarrow} W \text { bijection, } V \subseteq X, W \subseteq Y\}
$$

It is clear that

$$
X \mapsto X^{+}:=X \backslash\left\{0_{X}\right\}
$$

and

$$
\varphi \mapsto\left\{\varphi: X \backslash \varphi^{-1}\left(0_{Y}\right) \stackrel{\sim}{\longrightarrow} \varphi\left(X \backslash \varphi^{-1}\left(0_{Y}\right)\right)\right\}
$$

is an isomorphism of categories

$$
\mathbb{F}_{Y, X} \stackrel{\sim}{\longrightarrow} \mathbb{F}_{Y^{+}, X^{+}}^{\prime}
$$

We shall identify $\mathbb{F}$ with $\mathbb{F}^{\prime}$. Thus from now on the objects of $\mathbb{F}$ are finite sets without a distinguished zero element. Alternatively, $\mathbb{F}_{Y, X}$ are the $Y \times X$ matrices with entries 0,1 and with at most one 1 in every row and column.

We have a functor

$$
\oplus: \mathbb{F} \times \mathbb{F} \rightarrow \mathbb{F}
$$

given by the disjoint union of sets. More formally, for sets $X, Y$ we let

$$
X \oplus Y=\{(z, i) \mid i \in\{0,1\} ; i=0 \Rightarrow z \in X, i=1 \Rightarrow z \in Y\}
$$




\section{NON-ADDITIVE GEOMETRY}

and for $f_{0} \in \mathbb{F}_{X^{\prime}, X}, f_{1} \in \mathbb{F}_{Y^{\prime}, Y}$, we have $f_{0} \oplus f_{1} \in \mathbb{F}_{X^{\prime} \oplus Y^{\prime}, X \oplus Y}$ given by

$$
f_{0} \oplus f_{1}(z, i)=\left(f_{i}(z), i\right) .
$$

(Note that in the version of $\mathbb{F}$ where the objects have a distinguished zero element, $X \oplus Y$ is obtained from the disjoint union $X \coprod Y$ by identifying $0_{X}$ with $0_{Y}$.)

We have for $f_{0}^{\prime} \in \mathbb{F}_{X^{\prime \prime}, X^{\prime}}, f_{1}^{\prime} \in \mathbb{F}_{Y^{\prime \prime}, Y^{\prime}}$,

$$
\left(f_{0}^{\prime} \oplus f_{1}^{\prime}\right) \circ\left(f_{0} \oplus f_{1}\right)=\left(f_{0}^{\prime} \circ f_{0}\right) \oplus\left(f_{1}^{\prime} \circ f_{1}\right)
$$

and

$$
\operatorname{id}_{X} \oplus \operatorname{id}_{Y}=\operatorname{id}_{X \oplus Y} .
$$

The operation $\oplus$ makes $\mathbb{F}$ into a symmetric-monoidal category. The identity element is the empty set [0] (or the set with only the distinguished zero element), which is the initial and final object of the category $\mathbb{F}$. There are canonical isomorphisms in $\mathbb{F}$ :

$$
X \oplus[0] \stackrel{l_{X}}{\longleftarrow} X \stackrel{r_{X}}{\sim}[0] \oplus X .
$$

The commutativity isomorphism $c_{X, Y} \in \mathbb{F}_{Y \oplus X, X \oplus Y}$ is given by

$$
c_{X, Y}(z, i)=(z, 1-i) .
$$

The associativity isomorphism $a_{X, Y, Z} \in \mathbb{F}_{X \oplus(Y \oplus Z),(X \oplus Y) \oplus Z}$ is given by

$$
\begin{gathered}
a((w, 0), 0)=(w, 0), \\
a((w, 1), 0)=((w, 0), 1), \\
a(w, 1)=((w, 1), 1) .
\end{gathered}
$$

We shall usually abuse notation and view $l_{X}, r_{X}, c_{X, Y}, a_{X, Y, Z}$ as identifications; thus e.g. for $f_{i} \in$ $\mathbb{F}_{X_{i}^{\prime}, X_{i}}$ we write $f_{0} \oplus f_{1}=f_{1} \oplus f_{0}$ instead of

$$
c_{X_{0}^{\prime}, X_{1}^{\prime}} \circ\left(f_{0} \oplus f_{1}\right)=\left(f_{1} \oplus f_{0}\right) \circ c_{X_{0}, X_{1}} .
$$

We have a functor

$$
\otimes: \mathbb{F} \times \mathbb{F} \rightarrow \mathbb{F}
$$

given by the product of sets $X \otimes Y=\{(x, y) \mid x \in X, y \in Y\}$, and for $f_{0} \in \mathbb{F}_{X^{\prime}, X}, f_{1} \in \mathbb{F}_{Y^{\prime}, Y}$, we have $f_{0} \otimes f_{1} \in \mathbb{F}_{X^{\prime} \otimes Y^{\prime}, X \otimes Y}$ given by

$$
f_{0} \otimes f_{1}(x, y)=\left(f_{0}(x), f_{1}(y)\right) .
$$

(Note that working with the version of $\mathbb{F}$ where the objects have a distinguished zero element, $X \otimes Y$ is obtained from the product $X \times Y$ by identifying $\left(x, 0_{Y}\right)$ and $\left(0_{X}, y\right)$ with $\left(0_{X}, 0_{Y}\right)$ for all $x \in X, y \in Y$.)

We have for $f_{0}^{\prime} \in \mathbb{F}_{X^{\prime \prime}, X^{\prime}}, f_{1}^{\prime} \in \mathbb{F}_{Y^{\prime \prime}, Y^{\prime}}$,

$$
\left(f_{0}^{\prime} \otimes f_{1}^{\prime}\right) \circ\left(f_{0} \otimes f_{1}\right)=\left(f_{0}^{\prime} \circ f_{0}\right) \otimes\left(f_{1}^{\prime} \circ f_{1}\right)
$$

and

$$
\operatorname{id}_{X} \otimes \operatorname{id}_{Y}=\operatorname{id}_{X \otimes Y} .
$$

The operation $\otimes$ also makes $\mathbb{F}$ into a symmetric monoidal category. The identity element is the set with one element [1] (or the set with a distinguished zero element 0, and another element $[1]=\{0,1\})$. We have again isomorphisms in $\mathbb{F}$ :

$$
X \otimes[1] \stackrel{l_{X}^{*}}{\sim} X \stackrel{r_{X}^{*}}{\sim} X \otimes[1]
$$




\section{J. Shai HARAN}

and

$$
\begin{aligned}
c_{X, Y}^{*} \in \mathbb{F}_{Y \otimes X, X \otimes Y}, & c_{X, Y}^{*}(x, y)=(y, x), \\
a_{X, Y, Z}^{*} \in \mathbb{F}_{X \otimes(Y \otimes Z),(X \otimes Y) \otimes Z}, & a_{X, Y, Z}^{*}((x, y), z)=(x,(y, z)) .
\end{aligned}
$$

We have as well the distributivity isomorphism $d_{X_{0}, X_{1} ; Y} \in \mathbb{F}_{\left(X_{0} \otimes Y\right) \oplus\left(X_{1} \otimes Y\right),\left(X_{0} \oplus X_{1}\right) \otimes Y}$

$$
d_{X_{0}, X_{1} ; Y}((x, i), y)=((x, y), i), i \in\{0,1\} .
$$

We abuse notation and view $l_{X}^{*}, r_{X}^{*}, c_{X, Y}^{*}, a_{X, Y, Z}^{*}, d_{X_{0}, X_{1} ; Y}$ as identifications; thus e.g. for $f_{i} \in$ $\mathbb{F}_{X_{i}^{\prime}, X_{i}}, g \in \mathbb{F}_{Y^{\prime}, Y}$ we write

$$
\left(f_{0} \oplus f_{1}\right) \otimes g=\left(f_{0} \otimes g\right) \oplus\left(f_{1} \otimes g\right)
$$

which should be read as

$$
d_{X_{0}^{\prime}, X_{1}^{\prime} ; Y^{\prime}} \circ\left[\left(f_{0} \oplus f_{1}\right) \otimes g\right]=\left[\left(f_{0} \otimes g\right) \oplus\left(f_{1} \otimes g\right)\right] \circ d_{X_{0}, X_{1} ; Y} .
$$

We note that there is a natural involution

$$
\mathbb{F}_{Y, X} \stackrel{\sim}{\longrightarrow} \mathbb{F}_{X, Y}, f \mapsto f^{\mathrm{t}} .
$$

When viewing $\mathbb{F}_{Y, X}$ as the partial bijections $f: V \stackrel{\sim}{\longrightarrow} W, V \subseteq X$ and $W \subseteq Y, f^{\mathrm{t}}$ is the inverse bijection, $f^{t}=f^{-1}: W \stackrel{\sim}{\longrightarrow} V$. When we view $\mathbb{F}_{Y, X}$ as 0,1 matrices, $f^{t}$ is the transpose matrix.

We have

$$
\begin{gathered}
(g \circ f)^{\mathrm{t}}=f^{\mathrm{t}} \circ g^{\mathrm{t}}, \\
\left(\mathrm{id}_{X}\right)^{\mathrm{t}}=\mathrm{id}_{X}, \\
\left(f^{\mathrm{t}}\right)^{\mathrm{t}}=f, \\
\left(f_{0} \oplus f_{1}\right)^{\mathrm{t}}=f_{0}^{\mathrm{t}} \oplus f_{1}^{\mathrm{t}}, \\
\left(f_{0} \otimes f_{1}\right)^{\mathrm{t}}=f_{0}^{\mathrm{t}} \otimes f_{1}^{\mathrm{t}} .
\end{gathered}
$$

Remark. Whenever we use the notation for composition $f \circ g$ it will always be implicitly assumed that the domain of $f$ is the range of $g$; thus e.g. if we have $\left(f_{0} \oplus f_{1}\right) \circ g$ and $f_{i} \in \mathbb{F}_{X_{i}^{\prime}, X_{i}}$, it is implicitly assumed that $g$ has range $X_{0} \oplus X_{1}$.

\subsection{Variants $\mathbb{F}^{ \pm}$}

The model $\mathbb{F}$ for the field with one element is the one we shall use here, but there is a variant $\mathbb{F}^{ \pm}$which is important, and leads to a tighter theory. The objects of the category $\mathbb{F}^{ \pm}$are finite sets $X$ together with an action of the group $\{ \pm 1\}$, without fixed points (or with a unique fixed point - the zero element). A subset $X^{+} \subseteq X$ will be called a basis if $X$ is the disjoint union of $X^{+}$ and $-X^{+}=\left\{-x \mid x \in X^{+}\right\}$. The maps $f \in \mathbb{F}_{Y, X}^{ \pm}$are partial bijections

$$
f: V \stackrel{\sim}{\rightarrow} W, \quad V \subseteq X, \quad W \subseteq Y, \quad V=-V, \quad W=-W,
$$

that commute with the action $f(-x)=-f(x)$. Fixing basis $X^{+} \subseteq X, Y^{+} \subseteq Y$, we can identify the elements of $\mathbb{F}_{Y, X}^{ \pm}$with the $Y^{+} \times X^{+}$matrices of entries $0,1,-1$, with at most one non-zero term in each row and column. The map $f$ is identified with the matrix $\mathcal{M}(f)$, where for $x \in X^{+}, y \in Y^{+}$, $\mathcal{M}(f)_{y, x}=0$ (respectively, $1,-1$ ) if $f(x) \neq \pm y$ (respectively, $y,-y$ ).

We have functors

$$
\begin{gathered}
\oplus, \otimes: \mathbb{F}^{ \pm} \times \mathbb{F}^{ \pm} \rightarrow \mathbb{F}^{ \pm}, \\
X \oplus Y=\text { disjoint union of } X \text { and } Y \text {, with its natural }\{ \pm 1\} \text { action, } \\
X \otimes Y=X \times Y /_{(x, y) \sim(-x,-y)} \text {, with }\{ \pm 1\} \text { action }:-(x, y)=(-x, y)=(x,-y) .
\end{gathered}
$$




\section{NON-ADDITIVE GEOMETRY}

Write $x \otimes y$ for the image of $(x, y)$ in $X \otimes Y$; we have for $f_{0} \in \mathbb{F}_{X^{\prime}, X}^{ \pm}, f_{1} \in \mathbb{F}_{Y^{\prime}, Y}^{ \pm}$,

$$
f_{0} \otimes f_{1}(x \otimes y)=f_{0}(x) \otimes f_{1}(y) .
$$

The unit for $\oplus$ is [0], the initial and final object of $\mathbb{F}^{ \pm}$. The unit for $\otimes$ is $[ \pm 1]$. The analogue of formulas (1.5) to (1.19) remain true for $\mathbb{F}^{ \pm}$. We have an involution $\mathbb{F}^{ \pm} \rightarrow \mathbb{F}^{ \pm}, f \mapsto f^{\mathrm{t}}$, where $f^{\mathrm{t}}$ is the inverse bijection (or transpose of an $Y^{+} \times X^{+}$matrix), and formulas (1.21.1-5) remain true for $\mathbb{F}^{ \pm}$.

Definition. Let $X$ be an object of $\mathbb{F}^{ \pm}$and let $X^{+} \subseteq X$ be a basis. The number of elements of $X^{+}: d=\# X^{+}$will be called the dimension of $X$, and denoted $d=\operatorname{dim} X$.

For $n=1, \ldots, d$ let

$$
\begin{gathered}
\mathrm{P}^{n}(X)=\left\{x_{1} \otimes \cdots \otimes x_{n} \in X \otimes \cdots \otimes X \mid x_{i} \neq \pm x_{j} \text { for } i \neq j\right\} \\
\wedge^{n}(X)=\mathrm{P}^{n}(X) / \sim
\end{gathered}
$$

where $\sim$ is the equivalence relation

$$
x_{\sigma(1)} \otimes \cdots \otimes x_{\sigma(n)} \sim \operatorname{sgn}(\sigma) \cdot x_{1} \otimes \cdots \otimes x_{n}, \quad \text { for } \sigma \in S_{n} .
$$

Write $x_{1} \wedge \cdots \wedge x_{n}$ for the image of $x_{1} \otimes \cdots \otimes x_{n} \in \mathrm{P}^{n}(X)$ in $\wedge^{n}(X)$. A map $f \in \mathbb{F}_{Y, X}^{ \pm}$induces a map $\mathrm{P}^{n}(f) \in \mathbb{F}_{\mathrm{P}^{n}(Y), \mathrm{P}^{n}(X)}^{ \pm}$, which induces in turn a map $\wedge^{n}(f) \in \mathbb{F}_{\wedge^{n}(Y), \wedge^{n}(X)}^{ \pm}$,

$$
\wedge^{n}(f)\left(x_{1} \wedge \cdots \wedge x_{n}\right)=f\left(x_{1}\right) \wedge \cdots \wedge f\left(x_{n}\right) .
$$

For $n>d$ we have $\wedge^{n}(X)=[0]$, and by definition we let $\wedge^{0}(X)=[ \pm 1]$. Thus we have a sequence of functors

$$
\begin{gathered}
\wedge^{n}: \mathbb{F}^{ \pm} \rightarrow \mathbb{F}^{ \pm}, n=0,1, \ldots, \\
\wedge^{n}(f \circ g)=\wedge^{n}(f) \circ \wedge^{n}(g), \\
\wedge^{n}\left(\operatorname{id}_{X}\right)=\operatorname{id}_{\wedge^{n}(X)} \\
\wedge^{n}\left(f^{\mathrm{t}}\right)=\left(\wedge^{n}(f)\right)^{\mathrm{t}} .
\end{gathered}
$$

There are natural isomorphisms in $\mathbb{F}^{ \pm}$which we view as identifications

$$
\wedge^{n}(X \oplus Y)=\bigoplus_{0 \leqslant j \leqslant n} \wedge^{j}(X) \otimes \wedge^{n-j}(Y) .
$$

Remark 1.29. When we consider the objects $|\mathbb{F}|$ of the category $\mathbb{F}$ (respectively $\mathbb{F}^{ \pm}$), we assume that it contains $[n], n \geqslant 0$ (respectively $[ \pm n]$ ), and that it contains $X \oplus Y, X \otimes Y$ (respectively and $\left.\wedge^{n}(X)\right)$ whenever it contains $X, Y$. Hence we may assume $|\mathbb{F}|$ and $\left|\mathbb{F}^{ \pm}\right|$are countable sets. On the other hand, we shall not use the actual realization of $\mathbb{F}$ in most of what follows. All we need is a category $F$ with two symmetric monoidal structures $\oplus$ and $\otimes$, the unit element $[0]$ for $\oplus$ is the initial and final object of $F, \otimes$ is distributive over $\oplus$ and it respects $[0]: X \otimes[0]=[0]$. This opens up the possibility of introducing quantum deformations.

\subsection{The 'algebraic closure' $\overline{\mathbb{F}}$ of $\mathbb{F}$}

We can similarly work over the 'algebraic closure' $\overline{\mathbb{F}}$ of $\mathbb{F}$, which in arithmetic means adjoining all roots of unity $\mu \cong \mathbb{Q} / \mathbb{Z}$. The objects of $\overline{\mathbb{F}}$ are sets $X$ with $\mu$-action, satisfying the following two properties:

(i) set $X$ decomposes into a finite union of $\mu$-orbits

$$
X=X_{1} \sqcup \cdots \sqcup X_{d}, \quad X_{i}=\mu \cdot x_{i} ;
$$




\section{J. Shai HARAN}

(ii) for $x \in X$ there is a natural number $N$ and a finite set of primes $\left\{p_{1}, \ldots, p_{l}\right\}, p_{i} \nmid N$, such that the stabilizer of $x$ in $\mu$ is given by

$$
\{\zeta \in \mu \mid \zeta \cdot x=x\}=\mu_{N} \times \mu_{p_{1}^{\infty}} \times \cdots \times \mu_{p_{l}^{\infty}} .
$$

Here $\mu_{N}=\left\{\zeta \in \mu \mid \zeta^{N}=1\right\}$ and $\mu_{p^{\infty}}=\bigcup_{n} \mu_{p^{n}}$.

Let $x_{j} \in X, 1 \leqslant j \leqslant d$, be such that $X_{j}=\mu \cdot x_{j}$ for each $j$. Then the subset $X^{+}=\left\{x_{1}, \ldots, x_{d}\right\} \subseteq$ $X$ representing the $\mu$-orbits will be called a basis for $X$, and $d=\# X^{+}=\operatorname{dim} X$ the dimension of $X$. The maps in the category $\overline{\mathbb{F}}$ from an object $X$ to an object $Y$ are given by $\mu$-covariant partial bijections

$$
\overline{\mathbb{F}}_{Y, X}=\{f: V \stackrel{\sim}{\longrightarrow} W \mid V \subseteq X, W \subseteq Y, V=\mu \cdot V, W=\mu \cdot W, f(\zeta x)=\zeta f(x), \forall x \in V, \zeta \in \mu\} .
$$

We have functors

$$
\oplus, \otimes: \overline{\mathbb{F}} \times \overline{\mathbb{F}} \rightarrow \overline{\mathbb{F}}
$$

$X \oplus Y=$ disjoint union of $X$ and $Y$, with its natural $\mu$-action,

$X \otimes Y=X \times Y /_{(x, y) \sim\left(\zeta x, \zeta^{-1} y\right)}, \quad$ with $\mu$-action $\zeta \cdot(x, y)=(\zeta x, y)=(x, \zeta y)$.

We write $x \otimes y$ for the image of $(x, y)$ in $X \otimes Y$. For $f_{i} \in \overline{\mathbb{F}}_{Y_{i}, X_{i}}$ we have

$$
f_{0} \oplus f_{1} \in \overline{\mathbb{F}}_{Y_{0} \oplus Y_{1}, X_{0} \oplus X_{1}}, f_{0} \oplus f_{1}(z, i)=\left(f_{i}(z), i\right), \quad i=0,1,
$$

and we have

$$
f_{0} \otimes f_{1} \in \overline{\mathbb{F}}_{Y_{0} \otimes Y_{1}, X_{0} \otimes X_{1}}, f_{0} \otimes f_{1}\left(x_{0} \otimes x_{1}\right)=f_{0}\left(x_{0}\right) \otimes f_{1}\left(x_{1}\right) .
$$

Both $\oplus$ and $\otimes$ make $\overline{\mathbb{F}}$ into symmetric monoidal category; the unit for $\oplus$ is the empty set [0] which is the initial and final object of $\overline{\mathbb{F}}$; the unit for $\otimes$ is $[1]=\mu$. The analogue of formulas (1.5)-(1.19) remain true for $\overline{\mathbb{F}}$. We have an involution on $\overline{\mathbb{F}}$ satisfying $(1.21)$. We have $\lambda$-operations: for an object $X$ of $\overline{\mathbb{F}}$ of dimension $d=\operatorname{dim} X$ and for $n=1, \ldots, d$ we let

$$
\begin{gathered}
\mathrm{P}^{n}(X)=\left\{x_{1} \otimes \cdots \otimes x_{n} \in X \otimes \cdots \otimes X \mid x_{i} \neq \zeta x_{j} \text { for } i \neq j, \zeta \in \mu\right\}, \\
\wedge^{n}(X)=\mathrm{P}^{n}(X) / x_{\sigma(1)} \otimes \cdots \otimes x_{\sigma(n)} \sim \operatorname{sgn}(\sigma) \cdot x_{1} \otimes \cdots \otimes x_{n}, \sigma \in S_{n} .
\end{gathered}
$$

We write $x_{1} \wedge \cdots \wedge x_{n}$ for the image of $x_{1} \otimes \cdots \otimes x_{n}$ in $\wedge^{n}(X), x_{i} \in X$. A map $f \in \overline{\mathbb{F}}_{Y, X}$ induces a $\operatorname{map} \wedge^{n}(f) \in \overline{\mathbb{F}}_{\wedge^{n}(Y), \wedge^{n}(X)}$ by

$$
\begin{gathered}
\wedge^{n}(f)\left(x_{1} \wedge \cdots \wedge x_{n}\right)=f\left(x_{1}\right) \wedge \cdots \wedge f\left(x_{n}\right), \\
\operatorname{Domain}\left(\wedge^{n}(f)\right)=\left\{\left(x_{1} \wedge \cdots \wedge x_{n}\right) \mid x_{i} \in \operatorname{Domain}(f)\right\} .
\end{gathered}
$$

We let $\wedge^{0}(X)=[1]=\mu, \wedge^{1}(X)=X$, and $\wedge^{n}(X)=[0]$ for $n>\operatorname{dim} X$. Thus we have a sequence of functors $\wedge^{n}: \overline{\mathbb{F}} \rightarrow \overline{\mathbb{F}}, n=0,1,2, \ldots$, and (1.28) remains valid. A novelty of $\overline{\mathbb{F}}$ is that we have a sequence of functors given by Adam's operators

$$
\begin{gathered}
\psi^{n}: \overline{\mathbb{F}} \rightarrow \overline{\mathbb{F}}, \quad n= \pm 1, \pm 2, \ldots, \\
\psi^{n}(X)=\text { the set } X \text { with the new } \mu \text {-action } \zeta \cdot(n) x=\zeta^{n} \cdot x
\end{gathered}
$$

(we can take $n$ in $\left\{n=\left(n_{p}\right) \in \widehat{\mathbb{Z}}=\prod_{p} \mathbb{Z}_{p}, n_{p} \in \mathbb{Z}_{p}^{*}\right.$ for all but finitely many $\left.p\right\}$ ). These functors satisfy

$$
\begin{aligned}
& \psi^{n}(X \oplus Y)=\psi^{n}(X) \oplus \psi^{n}(Y), \\
& \psi^{n}(X \otimes Y)=\psi^{n}(X) \otimes \psi^{n}(Y),
\end{aligned}
$$

and are the analogue in our setting of the Frobenius endomorphisms in the theory of varieties over $\overline{\mathbb{F}_{q}}$. (Indeed, the action on $K$-theory of the Frobenius endomorphism for such varieties is given by $\psi^{q}$.) 


\section{NON-ADDITIVE GEOMETRY}

\section{2. $\mathbb{F}$-rings, variants, examples}

We give the definition of $\mathbb{F}$-rings and of $\mathcal{R}$ ing category. We give various variants of $\mathbb{F}$-rings with involution or with $\lambda$-ring structure. We then give our main examples.

\subsection{Definition of $\mathbb{F}$-rings}

Definition 2.1. An $\mathbb{F}$-ring is a category $A$ with objects the finite sets $|\mathbb{F}|$, and arrows $A_{Y, X}=$ $\operatorname{Hom}_{A}(X, Y)$ containing $\mathbb{F}_{Y, X}$, i.e. we have a faithful functor $\mathbb{F} \rightarrow A$ which is the identity on objects. We assume [0] is the initial and final object of $A$. We have two functors

$$
\oplus, \otimes: A \times A \rightarrow A,
$$

which agree with the given functors on $\mathbb{F}$, and which make $A$ into a symmetric monoidal category with the given identity $\left(l_{X}, r_{X} ; l_{X}^{*}, r_{X}^{*}\right)$, commutativity $\left(c_{X, Y} ; c_{X, Y}^{*}\right)$, associativity $\left(a_{X, Y, Z} ; a_{X, Y, Z}^{*}\right)$ isomorphisms of $\mathbb{F}$. We assume that $\otimes$ is distributive over $\oplus$ using the isomorphism $d_{X_{0}, X_{1} ; Y}$ of $\mathbb{F}$.

Thus in explicit terms, an $\mathbb{F}$-ring is a set

$$
A=\coprod_{Y, X \in|\mathbb{F}|} A_{Y, X}
$$

with operations

$$
\begin{gathered}
\circ: A_{Z, Y} \times A_{Y, X} \rightarrow A_{Z, X}, \\
\oplus: A_{Y_{0}, X_{0}} \times A_{Y_{1}, X_{1}} \rightarrow A_{Y_{0} \oplus Y_{1}, X_{0} \oplus X_{1}}, \\
\otimes: A_{Y_{0}, X_{0}} \times A_{Y_{1}, X_{1}} \rightarrow A_{Y_{0} \otimes Y_{1}, X_{0} \otimes X_{1}},
\end{gathered}
$$

satisfying

$$
\begin{gathered}
f \circ(g \circ h)=(f \circ g) \circ h ; \\
\operatorname{id}_{Y} \circ f=f=f \circ \mathrm{id}_{X}, \quad f \in A_{Y, X} ; \\
\left(f_{0} \oplus f_{1}\right) \circ\left(g_{0} \oplus g_{1}\right)=\left(f_{0} \circ g_{0}\right) \oplus\left(f_{1} \circ g_{1}\right), \quad g_{i} \in A_{Y_{i}, X_{i}}, f_{i} \in A_{Z_{i}, Y_{i}} ; \\
\operatorname{id}_{X} \oplus \mathrm{id}_{Y}=\mathrm{id}_{X \oplus Y} ; \\
f_{0} \oplus f_{1}=f_{1} \oplus f_{0} ; \\
f_{0} \oplus\left(f_{1} \oplus f_{2}\right)=\left(f_{0} \oplus f_{1}\right) \oplus f_{2} ; \\
f \oplus \mathrm{id}_{[0]}=f ; \\
\left(f_{0} \otimes f_{1}\right) \circ\left(g_{0} \otimes g_{1}\right)=\left(f_{0} \circ g_{0}\right) \otimes\left(f_{1} \circ g_{1}\right), \quad g_{i} \in A_{Y_{i}, X_{i}}, f_{i} \in A_{Z_{i}, Y_{i}} ; \\
\operatorname{id}_{X} \otimes \mathrm{id}_{Y}=\mathrm{id} \mathrm{d}_{X \otimes Y} ; \\
f_{0} \otimes f_{1}=f_{1} \otimes f_{0} ; \\
f_{0} \otimes\left(f_{1} \otimes f_{2}\right)=\left(f_{0} \otimes f_{1}\right) \otimes f_{2} ; \\
f \otimes \mathrm{id}_{[1]}=f ; \\
f \otimes\left(g_{0} \oplus g_{1}\right)=\left(f \otimes g_{0}\right) \oplus\left(f \otimes g_{1}\right) .
\end{gathered}
$$

Remark. We remind the reader that we omit the writing of the canonical isomorphisms of $\mathbb{F}$. Thus e.g (2.4.3) should be written

$$
\left(f_{0} \oplus f_{1}\right) \circ c_{X_{1}, X_{0}}=c_{Y_{1}, Y_{0}} \circ\left(f_{1} \oplus f_{0}\right), \quad f_{i} \in A_{Y_{i}, X_{i}} .
$$

We assume that $\mathbb{F}_{Y, X} \subseteq A_{Y, X}$, and that the above operations $\circ, \oplus, \otimes$ agree with the given operations on $\mathbb{F}$. In particular, we have the zero map $0_{Y, X} \in A_{Y, X}$, which is the unique map that factors through $[0]$. 


\section{J. SHAI HARAN}

We note that on $A_{[1],[1]}$, the operations of composition $\circ$ and of tensor product $\otimes$ induce the same operation, making $A_{[1],[1]}$ into a commutative monoid:

$$
f \cdot d \stackrel{\text { def }}{=} f \circ g=\left(f \otimes \operatorname{id}_{[1]}\right) \circ\left(\operatorname{id}_{[1]} \otimes g\right)=f \otimes g=g \otimes f, \quad f, g \in A_{[1],[1]} .
$$

The set $A_{[1],[1]}$ has a unit 1 coming from the map $\operatorname{id}_{[1],[1]}:[1] \rightarrow[1]$, and a zero element 0 coming from the map $0_{[1],[1]}:[1] \rightarrow[1], z \mapsto 0_{[1]}$. The set $A_{[1],[1]}$ acts on the sets $A_{Y, X}$,

$$
f \cdot g \stackrel{\text { def }}{=} f \otimes g, f \in A_{[1],[1]}, \quad g \in A_{Y, X} .
$$

This action satisfies

$$
\begin{gathered}
\left(f_{0} \cdot f_{1}\right) \cdot g=f_{0} \cdot\left(f_{1} \cdot g\right), f_{i} \in A_{[1],[1]}, \quad g \in A_{Y, X} ; \\
1 \cdot g=g ; \\
0 \cdot g=0_{Y, X} ; \\
f \cdot(g \circ h)=(f \cdot g) \circ h=g \circ(f \cdot h) ; \\
f \cdot\left(g_{0} \oplus g_{1}\right)=\left(f \cdot g_{0}\right) \oplus\left(f \cdot g_{1}\right) ; \\
f \cdot\left(g_{0} \otimes g_{1}\right)=\left(f \cdot g_{0}\right) \otimes g_{1}=g_{0} \otimes\left(f \cdot g_{1}\right) .
\end{gathered}
$$

Definition 2.9. Let $A, B$ be $\mathbb{F}$-rings. A functor $\varphi: A \rightarrow B$ is a homomorphism of $\mathbb{F}$-rings if

$$
\begin{gathered}
\varphi\left(A_{Y, X}\right) \subseteq B_{Y, X}, \\
\varphi(f)=f \text { for } f \in \mathbb{F}_{Y, X}, \\
\varphi(f \circ g)=\varphi(f) \circ \varphi(g), \\
\varphi\left(f_{0} \oplus f_{1}\right)=\varphi\left(f_{0}\right) \oplus \varphi\left(f_{1}\right), \\
\varphi\left(f_{0} \otimes f_{1}\right)=\varphi\left(f_{0}\right) \otimes \varphi\left(f_{1}\right) .
\end{gathered}
$$

Thus $\varphi$ is a functor over $\mathbb{F}$ that respects $\oplus$ and $\otimes$. It is clear that if $\varphi: A \rightarrow B, \psi: B \rightarrow C$ are homomorphisms of $\mathbb{F}$-rings, then $\psi \circ \varphi$ is a homomorphism of $\mathbb{F}$-rings, hence we have a category $\mathbb{F}$ - $\mathcal{R}$ ings, with $\mathbb{F}$ as an initial object.

Remark. A (commutative) ring category $A$ is a category with a symmetric monoidal structure

$$
\oplus: A \times A \rightarrow A
$$

with associativity (respectively commutativity, unit) isomorphisms $a$ (respectively, $c, u$ ), with the unit object for $\oplus$, denoted by [0], being the initial and final object of $A$, and another symmetric monoidal structure

$$
\otimes: A \times A \rightarrow A
$$

with associativity (respectively commutativity, unit) isomorphisms $a^{*}$ (respectively, $c^{*}, u^{*}$ ), the unit object for $\otimes$ is denoted by [1], and distributive isomorphisms

$$
d_{Y ; X_{0}, X_{1}}: Y \otimes\left(X_{0} \oplus X_{1}\right) \stackrel{\sim}{\longrightarrow}\left(Y \otimes X_{0}\right) \oplus\left(Y \otimes X_{1}\right)
$$

functorial in $Y, X_{0}, X_{1} \in|A|$, and compatible with $a, c, u, a^{*}, c^{*}, u^{*}$. That is, we have commutative diagrams. For $X_{i}, Y_{i}$ objects of $A$, we have the following. 
$(a)$

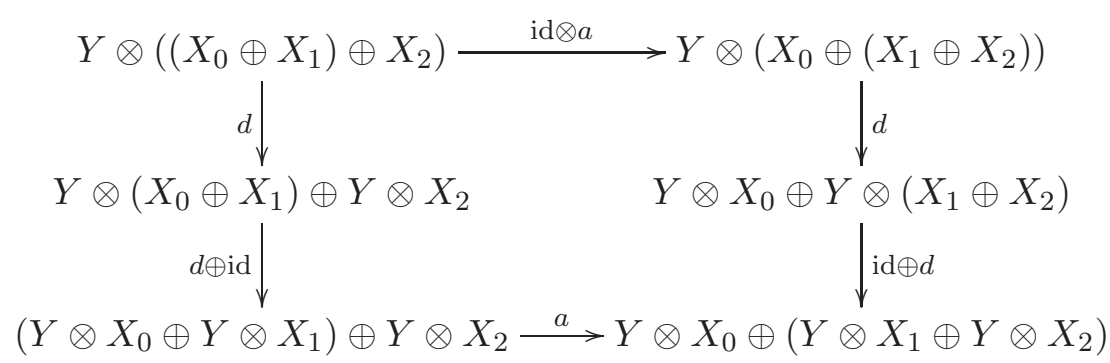

$\left(a^{*}\right)$

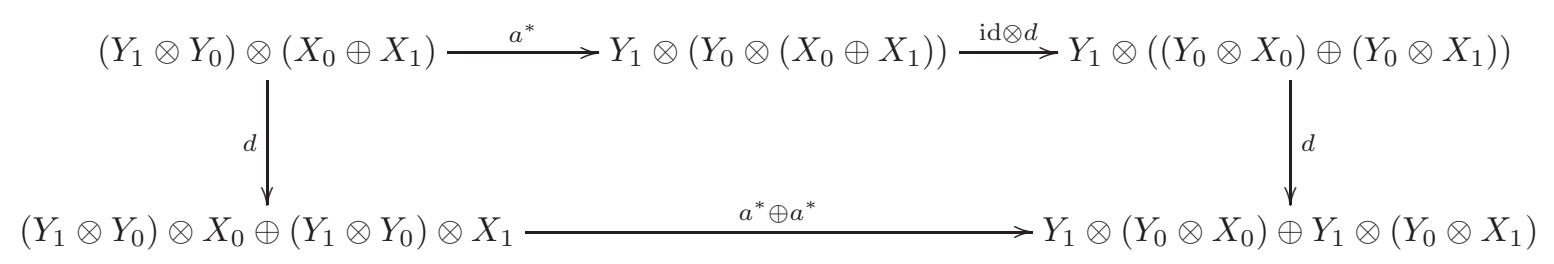

(c)

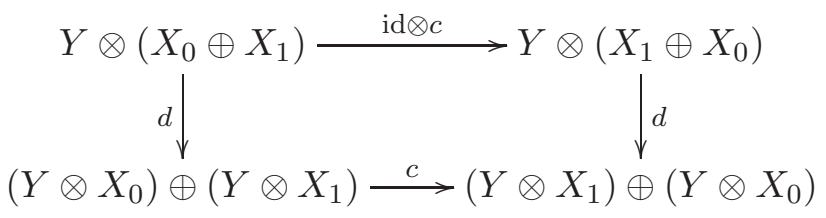

$\left(u^{*}\right)$ With [1] denoting the unit object for $\otimes$,

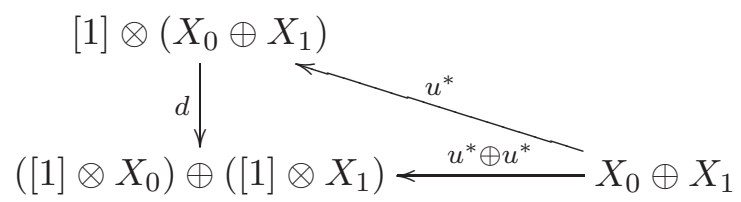

$(u)$ The canonical map gives isomorphism $Y \otimes[0] \stackrel{\sim}{\rightarrow}[0]$, and we have

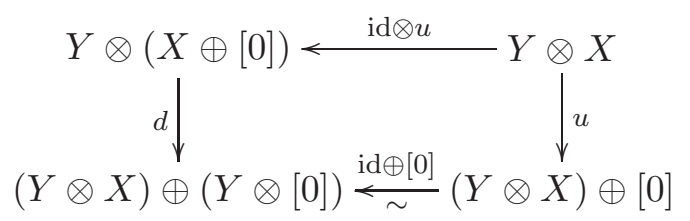

A homomorphism of commutative ring categories $\varphi: A \rightarrow A^{\prime}$ is a functor respecting $\oplus, a, c, u, \otimes$, $a^{*}, c^{*}, u^{*}, d$. Thus an $\mathbb{F}$-ring is a homomorphism of commutative ring categories $\varphi: \mathbb{F} \rightarrow A$ which is a bijection on objects. Most of what we do in the following works more generally for commutative ring categories, but working with $\mathbb{F}$-rings is easier and allows the suppression of the isomorphisms $a, c, u, a^{*}, c^{*}, u^{*}, d$. On the other hand it will be interesting to work more generally with braided ring categories, replacing the symmetric monoidal structure $\otimes$ by a braided monoidal structure; this might lead to the quantum geometry behind [Har01] and [Har06].

$2.2 \mathbb{F}^{\mathrm{t}}, \mathbb{F}^{ \pm}, \mathbb{F}^{\lambda}, \overline{\mathbb{F}}, \overline{\mathbb{F}}^{\lambda}$-rings

Remark 2.10. We can define $\mathbb{F}^{\mathrm{t}}$-rings to be $\mathbb{F}$-rings with involution

$$
A_{Y, X} \rightarrow A_{X, Y}, \quad f \mapsto f^{\mathrm{t}},
$$




\section{J. SHAI HARAN}

agreeing with the given involution on $\mathbb{F}$, and satisfying

$$
\begin{aligned}
(f \circ g)^{\mathrm{t}} & =g^{\mathrm{t}} \circ f^{\mathrm{t}}, \\
f^{\mathrm{tt}} & =f, \\
\left(f_{0} \oplus f_{1}\right)^{\mathrm{t}} & =f_{0}^{\mathrm{t}} \oplus f_{1}^{\mathrm{t}}, \\
\left(f_{0} \otimes f_{1}\right)^{\mathrm{t}} & =f_{0}^{\mathrm{t}} \otimes f_{1}^{\mathrm{t}} .
\end{aligned}
$$

A homomorphism of $\mathbb{F}^{\mathrm{t}}$-rings is a homomorphism of $\mathbb{F}$-rings $\varphi$ satisfying

$$
\varphi(f)^{\mathrm{t}}=\varphi\left(f^{\mathrm{t}}\right) .
$$

Thus we have a category of $\mathbb{F}^{\mathrm{t}}-\mathcal{R}$ ings.

Remark 2.11. One defines $\mathbb{F}^{ \pm}$-rings $A$ as a category with objects $\left|\mathbb{F}^{ \pm}\right|$, with $[0]$ as an initial and final object, and with symmetric monoidal structures

$$
\oplus, \otimes: A \times A \rightarrow A,
$$

with [0], $[ \pm 1]$ as identities, with $\otimes$ distributive over $\oplus$, and with a functor $\mathbb{F}^{ \pm} \rightarrow A$ which is the identity on objects and respects the symmetric monoidal structures $\oplus$ and $\otimes$. A homomorphism $\varphi: A \rightarrow B$ of $\mathbb{F}^{ \pm}$-rings is a functor over $\mathbb{F}^{ \pm}$which respects the symmetric monoidal structures $\oplus$ and $\otimes$. Thus we have the category $\mathbb{F}^{ \pm}$-R ings. Replacing $\mathbb{F}^{ \pm}$by $\overline{\mathbb{F}}$, and $[ \pm 1]$ by $\mu$, one obtains the definition of the category $\overline{\mathbb{F}}$-R ings. We can similarly define $\mathbb{F}^{ \pm, t}$-rings to be $\mathbb{F}^{ \pm}$-rings with involution, agreeing with the given involution on $\mathbb{F}^{ \pm}$, and respecting $\oplus$ and $\otimes$. Maps of $\mathbb{F}^{ \pm, t}$-rings are maps of $\mathbb{F}^{ \pm}$-rings respecting the involution, hence we have a category $\mathbb{F}^{ \pm, \mathrm{t}}-\mathcal{R}$ ings. Similarly we have the category $\overline{\mathbb{F}}^{\mathrm{t}}$ - Rings.

Definition 2.12. An $\mathbb{F}^{\lambda}$-ring $A$ is an $\mathbb{F}^{ \pm}$-ring, together with functors

$$
\wedge^{k}: A \rightarrow A, \quad k=0,1, \ldots
$$

such that

$$
\begin{gathered}
\wedge^{k}: A_{Y, X} \rightarrow A_{\wedge^{k}(Y), \wedge^{k}(X)} \\
\wedge^{k}\left(\operatorname{id}_{X}\right)=\operatorname{id}_{\wedge^{k}(X)}
\end{gathered}
$$

and moreover $\wedge^{k}$ agree with the given operation on $\mathbb{F}^{ \pm}$cf. (1.26), and

$$
\begin{gathered}
\wedge^{0}(f)=1, \\
\wedge^{1}(f)=f, \\
\wedge^{k}(f \oplus g)=\bigoplus_{0 \leqslant j \leqslant k} \wedge^{j}(f) \otimes \wedge^{k-j}(g) .
\end{gathered}
$$

One similarly defines an $\mathbb{F}^{\lambda, t}$-ring to be an $\mathbb{F}^{ \pm, t}$-ring and an $\mathbb{F}^{\lambda}$-ring such that

$$
\wedge^{k}(f)^{\mathrm{t}}=\wedge^{k}\left(f^{\mathrm{t}}\right)
$$

Similarly replacing $\mathbb{F}^{ \pm}$by $\overline{\mathbb{F}}$ one defines an $\overline{\mathbb{F}}^{\lambda}$-ring to be an $\overline{\mathbb{F}}$-ring $A$ together with functors $(2.12 .1)$ satisfying (2.12.2)-(2.12.6). Similarly, $\overline{\mathbb{F}}^{\lambda, t}$-rings are $\overline{\mathbb{F}}^{\lambda}$-rings with involution satisfying (2.12.7).

Remark. It is possible to add further axioms (e.g. the ones corresponding to 'special' $\lambda$-rings). Here we shall only note the following. For $X$ a finite set with $\{ \pm\}$ action, an orientation on $X$ is a choice of an isomorphism

$$
\varepsilon:[ \pm 1] \stackrel{\sim}{\longrightarrow} \wedge^{d}(X), \quad d=\operatorname{dim} X
$$


i.e. it is a choice of one of the two (non-zero) elements $\varepsilon(1) \in \wedge^{d}(X)$. For $A$ an $\mathbb{F}^{\lambda}$-ring, and for $a \in A_{X, X}$ we have $\operatorname{det}_{X}(a) \in A_{[ \pm 1],[ \pm 1]}$, defined by

$$
\operatorname{det}_{X}(a)=\varepsilon^{-1} \circ \wedge^{d}(a) \circ \varepsilon .
$$

It is independent of the choice of $\varepsilon \in \mathbb{F}_{\wedge^{d}(X),[ \pm 1]} \subseteq A_{\wedge^{d}(X),[ \pm 1]}$, and it satisfies

$$
\begin{gathered}
\operatorname{det}_{X}\left(a \circ a^{\prime}\right)=\operatorname{det}_{X}(a) \cdot \operatorname{det}_{X}\left(a^{\prime}\right), \\
\operatorname{det}_{X}\left(\operatorname{id}_{X}\right)=1\left(=\operatorname{id}_{[ \pm 1]}\right), \\
\operatorname{det}_{X_{1} \oplus X_{2}}\left(a_{1} \oplus a_{2}\right)=\operatorname{det}_{X_{1}}\left(a_{1}\right) \cdot \operatorname{det}_{X_{2}}\left(a_{2}\right) .
\end{gathered}
$$

The choice of the orientation $\varepsilon$ on $X$ gives also the duality isomorphism

$$
\widetilde{\varepsilon}: X \stackrel{\sim}{\longrightarrow} \wedge^{d-1}(X) \quad\left(\text { and } \widetilde{\varepsilon}: \wedge^{j}(X) \stackrel{\sim}{\longrightarrow} \wedge^{d-j}(X)\right),
$$

uniquely determined by

$$
x \wedge \widetilde{\varepsilon}(x)=\varepsilon(1), \quad x \in X .
$$

For $a \in A_{X, X}$, we have $a^{\text {adj }} \in A_{X, X}$, defined by

$$
a^{\text {adj }}=\widetilde{\varepsilon}^{-1} \circ \wedge^{d-1}\left(a^{\mathrm{t}}\right) \circ \widetilde{\varepsilon},
$$

where $\widetilde{\varepsilon} \in \mathbb{F}_{\wedge^{d-1}(X), X} \subseteq A_{\wedge^{d-1}(X), X}$. It is independent of the choice of $\varepsilon$, and it satisfies

$$
\begin{gathered}
(a \circ b)^{\text {adj }}=b^{\text {adj }} \circ a^{\text {adj }}, \\
\left(\operatorname{id}_{X}\right)^{\text {adj }}=\operatorname{id}_{X} .
\end{gathered}
$$

It is useful to have the expansion of the determinant by rows/columns,

$$
a \circ a^{\operatorname{adj}}=a^{\operatorname{adj}} \circ a=\operatorname{det}(a) \cdot \operatorname{id}_{X} .
$$

As a corollary of (2.12.19) we have that $a \in A_{X, X}$ is invertible (i.e. there exists $a^{-1} \in A_{X, X}$ with $\left.a \circ a^{-1}=a^{-1} \circ a=\mathrm{id}_{X}\right)$ if and only if $\operatorname{det}_{X}(a) \in A_{[ \pm 1],[ \pm 1]}$ is invertible. Indeed, if $a$ is invertible $\operatorname{det}(a)$ is always invertible with inverse $\operatorname{det}\left(a^{-1}\right)$, and conversely, if $\operatorname{det}(a)$ is invertible then by (2.12.19) $a$ itself is invertible with inverse $\operatorname{det}(a)^{-1} \cdot a^{\text {adj }}$.

Remark. For an $\mathbb{F}$-ring $A$ (or an $\mathbb{F}^{ \pm}$or $\overline{\mathbb{F}}$-ring), we let $G L_{X}(A)$ denote the group of invertible elements in $A_{X, X}$,

$$
G L_{X}(A)=\left\{a \in A_{X, X} \mid \exists a^{-1} \in A_{X, X}, a \circ a^{-1}=a^{-1} \circ a=\operatorname{id}_{X}\right\} .
$$

We have homomorphisms,

$$
\begin{aligned}
& G L_{X_{1}}(A) \times G L_{X_{2}}(A) \rightarrow G L_{X_{1} \oplus X_{2}}(A),\left(a_{1}, a_{2}\right) \mapsto a_{1} \oplus a_{2}, \\
& G L_{X_{1}}(A) \times G L_{X_{2}}(A) \rightarrow G L_{X_{1} \otimes X_{2}}(A), \quad\left(a_{1}, a_{2}\right) \mapsto a_{1} \otimes a_{2} .
\end{aligned}
$$

In particular, we have the homomorphisms

$$
G L_{[n]}(A) \rightarrow G L_{[n+1]}(A), \quad a \mapsto a \oplus \operatorname{id}_{[1]},
$$

hence the direct limit

$$
G L_{\infty}(A)=\lim _{\longrightarrow} G L_{[n]}(A) .
$$

We can then define the higher $K$-groups of $A$ following Quillen [Qui73]:

$$
K_{n}(A)=\pi_{n+1}\left(B G L_{\infty}(A)^{+}\right) .
$$

Note that for an $\mathbb{F}$-ring $A$ associated with a commutative ring $B, A=\mathbb{F}(B)$ (see example 1 below), we have $G L_{\infty}(A)=\underline{\lim _{\longrightarrow}} G L_{n}(B)$ and $K_{n}(A)=K_{n}(B)$. 


\section{J. SHAi HARAN}

\subsection{Examples of $\mathbb{F}$-rings}

Example 0. $\mathbb{F}$ is an $\mathbb{F}$-ring.

Example 1. Let $A$ be a commutative ring (always with identity). We denote by $\mathbb{F}(A)$ the $\mathbb{F}$-ring with

$$
\mathbb{F}(A)_{Y, X}=\operatorname{Hom}_{A}(A \cdot X, A \cdot Y)=Y \times X \text { - matrices with values in } A,
$$

where $\circ$ is the usual composition of $A$-linear homomorphisms (or multiplication of $A$-valued matrices), and where $\oplus$ is the usual direct sum, and $\otimes$ the tensor product. Note that a homomorphism of commutative rings $\varphi: A \rightarrow B$ induces a map of $\mathbb{F}$-rings $\mathbb{F}(\varphi): \mathbb{F}(A) \rightarrow \mathbb{F}(B)$, hence we have a functor

$$
\mathbb{F}: \mathcal{R} \text { ings } \rightarrow \mathbb{F} \text {-R ings. }
$$

Moreover let $\varphi: \mathbb{F}(A) \rightarrow \mathbb{F}(B)$ be a map of $\mathbb{F}$-rings. For $a \in \mathbb{F}(A)_{Y, X}$ write

$$
a_{y, x}=j_{y}^{\mathrm{t}} \circ a \circ j_{x} \in A=\mathbb{F}(A)_{[1],[1]}
$$

for its matrix coefficients, where $j_{x}, j_{y}^{\mathrm{t}}$ are the morphisms of $\mathbb{F}$ given by

$$
j_{x}:[1] \rightarrow X, \quad j_{x}(1)=x \in X,
$$

and where

$$
j_{y}^{\mathrm{t}}: Y \rightarrow[1] \text { is the partial bijection }\{y\} \rightarrow\{1\} .
$$

Since $\varphi$ is a functor over $\mathbb{F}$, and $j_{y}^{\mathrm{t}}, j_{x} \in \mathbb{F}$, we have $\varphi(a)_{y, x}=\varphi\left(a_{y, x}\right)$ and $\varphi$ is determined by $\varphi: A=\mathbb{F}(A)_{[1],[1]} \rightarrow B=\mathbb{F}(B)_{[1],[1]}$. This map is multiplicative, $\varphi\left(a_{1} \cdot a_{2}\right)=\varphi\left(a_{1}\right) \cdot \varphi\left(a_{2}\right), \varphi(1)=1$, and moreover it is additive,

$$
\varphi\left(a_{1}+a_{2}\right)=\varphi\left[\left(a_{1}, a_{2}\right) \circ\left(\begin{array}{l}
1 \\
1
\end{array}\right)\right]=\left(\varphi\left(a_{1}\right), \varphi\left(a_{2}\right)\right) \circ\left(\begin{array}{l}
1 \\
1
\end{array}\right)=\varphi\left(a_{1}\right)+\varphi\left(a_{2}\right) .
$$

Thus the functor $\mathbb{F}$ is fully faithful.

Example 2. Let $M$ be a commutative monoid with a unit 1 and a zero element 0 . Thus we have an associative and commutative operation

$$
\begin{gathered}
M \times M \rightarrow M, \quad(a, b) \mapsto a \cdot b, \\
a \cdot(b \cdot c)=(a \cdot b) \cdot c, \quad a \cdot b=b \cdot a,
\end{gathered}
$$

and $1 \in M$ is the (unique) element such that

$$
a \cdot 1=a, \quad a \in M,
$$

and $0 \in M$ is the (unique) element such that

$$
a \cdot 0=0, \quad a \in M .
$$

Let $\mathbb{F}\langle M\rangle$ denote the $\mathbb{F}$-ring with $\mathbb{F}\langle M\rangle_{Y, X}$ the $Y \times X$ matrices with values in $M$ with at most one non-zero entry in every row and column. Note that this is indeed an $\mathbb{F}$-ring with the usual 'multiplication' of matrices $\circ$ (there is no addition involved - only multiplication in $M$ ), direct sum $\oplus$, and tensor product $\otimes$.

Denoting by $\mathcal{M o n}_{0,1}$ the category of commutative monoids with unit and zero elements, and with maps respecting the operation and the elements 0,1 , the above construction yields a functor

$$
\text { Mon }_{0,1} \rightarrow \mathbb{F} \text { - Rings, } \quad M \mapsto \mathbb{F}\langle M\rangle .
$$

This is the functor left-adjoint to the functor

$$
\mathbb{F} \text {-Rings } \rightarrow \mathcal{M o n}_{0,1}, \quad A \mapsto A_{[1],[1]},
$$


namely

$$
\operatorname{Hom}_{\mathbb{F}-\mathcal{R} \text { ings }}(\mathbb{F}\langle M\rangle, A)=\operatorname{Hom}_{\mathcal{M} n_{0,1}}\left(M, A_{[1],[1]}\right) .
$$

As a particular example, take $M=M_{q}$ to be the free monoid (with zero) generated by one element $q$,

$$
M_{q}=q^{\mathbb{N}} \cup\{0\} .
$$

Then

$$
\operatorname{Hom}_{\mathbb{F}-\mathcal{R} \text { ings }}\left(\mathbb{F}\left\langle M_{q}\right\rangle, A\right)=A_{[1],[1]} .
$$

Example 3. Let $\mathbb{S}$ denote the $\mathbb{F}$-ring of sets. The objects of $\mathbb{S}$ are the finite sets of $|\mathbb{F}|$, and we let $\mathbb{S}_{Y, X}$ be the partially defined maps of sets from $X$ to $Y$,

$$
\mathbb{S}_{Y, X}=\{f: V \rightarrow Y \mid V \subseteq X\} .
$$

Notice that if $A$ is an $\mathbb{F}$-ring, the opposite category $A^{\text {op }}$ is again an $\mathbb{F}$-ring, since $\mathbb{F}^{\text {op }}=\mathbb{F}$ and since the axioms of an $\mathbb{F}$-ring are self-dual. In particular, we have the $\mathbb{F}$-ring $\mathbb{S}^{\text {op }}$ with

$$
\mathbb{S}_{Y, X}^{\mathrm{pp}}=\{f: V \rightarrow X \mid V \subseteq Y\}
$$

We have the $\mathbb{F}$-ring of relations $\mathcal{R}$ that contains both $\mathbb{S}$ and $\mathbb{S}^{\text {op }}$, with

$$
\mathcal{R}_{Y, X}=\{F \subseteq Y \times X \text { a subset }\} .
$$

The composition of $F \in \mathcal{R}_{Y, X}$ and $G \in \mathcal{R}_{Z, Y}$ is given by

$$
G \circ F=\{(z, x) \in Z \times X \mid \exists y \in Y \text { with }(z, y) \in G,(y, x) \in F\},
$$

and $G \circ F \in \mathcal{R}_{Z, X}$.

The sum $F_{0} \oplus F_{1} \in \mathcal{R}_{Y_{0} \oplus Y_{1}, X_{0} \oplus X_{1}}$ of $F_{i} \in \mathcal{R}_{Y_{i}, X_{i}}$ is given by the disjoint union of $F_{0}$ and $F_{1}$,

$$
F_{0} \oplus F_{1}=\left\{((x, i),(y, i)) \mid(x, y) \in F_{i}\right\},
$$

and the product $F_{0} \otimes F_{1} \in \mathcal{R}_{Y_{0} \otimes Y_{1}, X_{0} \otimes X_{1}}$ is given by

$$
F_{0} \otimes F_{1}=\left\{\left(\left(x_{0}, x_{1}\right),\left(y_{0}, y_{1}\right)\right) \mid\left(x_{0}, y_{0}\right) \in F_{0},\left(x_{1}, y_{1}\right) \in F_{1}\right\} .
$$

Equivalently, $\mathcal{R}_{Y, X}$ are the $Y \times X$ matrices with values in $\{0,1\}$, and $\oplus, \otimes$ are the direct sum and the tensor product of matrices (but $\circ$ does not correspond to matrix multiplication).

We have the $\mathbb{F}$-subring of $\mathbb{F}(\mathbb{Z})$ consisting of matrices with values in $\mathbb{N}$; we denote it by $\mathbb{F}(\mathbb{N})$. This $\mathbb{F}$-ring also contains $\mathbb{S}$ and $\mathbb{S}^{\text {op }}$, but composition in $\mathbb{F}(\mathbb{N})$ is matrix multiplication. We can summarize these basic $\mathbb{F}$-rings in the following diagram, where $A$ is a commutative ring.

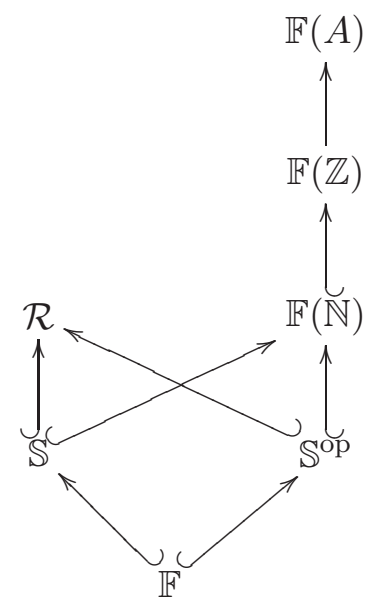




\section{J. SHAI HARAN}

Example 4. Let $\mathbb{k}$ be a ring and $\eta: \mathbb{k} \rightarrow \mathbb{C}$ an embedding (e.g. $\eta$ a real or complex prime of a number field). For $X \in|\mathbb{F}|$, let $\mathbb{k} \cdot X$ denote the free $\mathbb{k}$-module with inner product having $X$ as an orthonormal basis. Thus for $a=\left(a_{x}\right) \in \mathbb{k} \cdot X$ we have its norm

$$
|a|_{\eta}=\sqrt{\left(\sum_{x \in X}\left|\eta\left(a_{x}\right)\right|^{2}\right)}
$$

and for a $\mathbb{k}$-linear map $f \in \operatorname{Hom}_{\mathbb{k}}(\mathbb{k} \cdot X, \mathbb{k} \cdot Y)$ we have its operator norm

$$
|f|_{\eta}=\sup _{|a|_{\eta} \leqslant 1}|f(a)|_{\eta}
$$

We have

$$
\begin{gathered}
|f \circ g|_{\eta} \leqslant|f|_{\eta} \cdot|g|_{\eta}, \\
|f \oplus g|_{\eta}=\max \left\{|f|_{\eta},|g|_{\eta}\right\} \\
|f \otimes g|_{\eta}=|f|_{\eta} \cdot|g|_{\eta} .
\end{gathered}
$$

Let $\mathcal{O}_{\mathbb{k}, \eta}$ denote the $\mathbb{F}$-ring with

$$
\left(\mathcal{O}_{\mathbb{k}, \eta}\right)_{Y, X}=\left\{f \in \operatorname{Hom}_{\mathbb{k}}(\mathbb{k} \cdot X, \mathbb{k} \cdot Y),|f|_{\eta} \leqslant 1\right\}
$$

and with the usual operations $\circ, \oplus, \otimes$.

For $X_{0}, X_{1} \in|\mathbb{F}|$, denote by

$$
j_{i}: X_{i} \rightarrow X_{0} \oplus X_{1}, \quad j_{i}(x)=(x, i),
$$

the natural inclusion, and by

$$
j_{i}^{\mathrm{t}}: X_{0} \oplus X_{1} \rightarrow X_{i}, \quad j_{i}^{\mathrm{t}}\left(x, i^{\prime}\right)= \begin{cases}x, & i=i^{\prime} \\ 0, & i \neq i^{\prime}\end{cases}
$$

its transpose. For an $\mathbb{F}$-ring $A$ we get maps

$$
A_{Y, X_{0} \oplus X_{1}} \rightarrow A_{Y, X_{0}} \times A_{Y, X_{1}}, \quad f \mapsto\left(f \circ j_{0}, f \circ j_{1}\right),
$$

and

$$
A_{X_{0} \oplus X_{1}, Y} \rightarrow A_{X_{0}, Y} \times A_{X_{1}, Y}, \quad f \mapsto\left(j_{0}^{\mathrm{t}} \circ f, j_{1}^{\mathrm{t}} \circ f\right) .
$$

We say that $A$ is a matrix ring if these maps are always injections. Equivalently, $A$ is a matrix ring if every element is determined by its coefficients, that is we have an injection

$$
A_{Y, X} \hookrightarrow\left(A_{[1],[1]}\right)^{Y \times X}, \quad f \mapsto\left\{j_{y}^{\mathrm{t}} \circ f \circ j_{x}\right\}_{y \in Y, x \in X}
$$

with $j_{x}, j_{y}^{\mathrm{t}}$ as in $(2.13 .2)$ and $(2.13 .3)$.

The above examples $0,1,2,3$ (except for $\mathcal{R}$ ) and 4 , all constitute matrix rings. The following gives examples of $\mathbb{F}$-rings which are not matrix rings (they are the 'residue $\mathbb{F}$-field' of the $\mathbb{F}$-rings of Example $4(2.16))$.

Example 5. Let $\mathbb{k}$ be a ring and let $\eta: \mathbb{k} \rightarrow \mathbb{C}$ be an embedding, and for $X \in|\mathbb{F}|$, let $\mathbb{k} \cdot X$ denote the free $\mathbb{k}$-module with basis $X$ and with the inner product having $X$ as an orthonormal basis. Let $\mathbb{F}_{\mathbb{k}, \eta}$ denote the $\mathbb{F}$-ring of 'partial isometries', with

$$
\begin{aligned}
\left(\mathbb{F}_{\mathbb{k}, \eta}\right)_{Y, X}=\{f: V \stackrel{\sim}{\longrightarrow} W, \text { with } V \subseteq \mathbb{k} \cdot X, W \subseteq \mathbb{k} \cdot Y \\
\mathbb{k} \text {-submodules and } f \text { is a } \mathbb{k} \text {-linear isometry }\} .
\end{aligned}
$$

For $f=(f: V \stackrel{\sim}{\longrightarrow} W) \in\left(\mathbb{F}_{\mathbb{k}, \eta}\right)_{Y, X}, g=\left(g: W^{\prime} \stackrel{\sim}{\longrightarrow} U\right) \in\left(\mathbb{F}_{\mathbb{k}, \eta}\right)_{Z, Y}$, we have

$$
g \circ f=\left(g \circ f: f^{-1}\left(W \cap W^{\prime}\right) \stackrel{\sim}{\longrightarrow} g\left(W \cap W^{\prime}\right)\right) \in\left(\mathbb{F}_{\mathbb{k}, \eta}\right)_{Z, X} ;
$$




\section{NON-ADDITIVE GEOMETRY}

and for $f_{i}=\left(f_{i}: V_{i} \stackrel{\sim}{\longrightarrow} W_{i}\right) \in\left(\mathbb{F}_{\mathbb{k}, \eta}\right)_{Y_{i}, X_{i}}$, we have

$$
\begin{aligned}
& f_{0} \oplus f_{1}=\left(f_{0} \oplus f_{1}: V_{0} \oplus V_{1} \stackrel{\sim}{\longrightarrow} W_{0} \oplus W_{1}\right), \\
& f_{0} \otimes f_{1}=\left(f_{0} \otimes f_{1}: V_{0} \otimes V_{1} \stackrel{\sim}{\longrightarrow} W_{0} \otimes W_{1}\right) .
\end{aligned}
$$

We will see in Example 4.21 below that $\mathbb{F}_{\mathbb{k}, \eta}$ is indeed the residue field of $\mathcal{O}_{\mathbb{k}, \eta}$.

Remark. All of the above examples (except $\mathbb{S}$ and $\mathbb{S}^{\text {op }}$ ) have a natural involution making them into $\mathbb{F}^{t}$-rings. Moreover, all the examples have obvious analogous $\mathbb{F}^{ \pm}$-rings. For example, for a commutative ring $A$, we have the $\mathbb{F}^{ \pm}$-ring $\mathbb{F}^{ \pm}(A)$ with

$$
\mathbb{F}^{ \pm}(A)_{Y, X}=\operatorname{Hom}_{A}(A \cdot X, A \cdot Y),
$$

where $A \cdot X$ denotes the free $A$-module with basis $\{(x) \mid x \in X\}$, divided by the $A$-submodule generated by $\{(x)+(-x) \mid x \in X\}$ :

$$
A \cdot X=\bigoplus_{x \in X} A(x) /(-x) \sim-(x) ;
$$

alternatively, $A \cdot X$ is the free $A$-module with basis $X^{+}$, where $X^{+} \subseteq X$ is a basis of the \pm 1 -set $X$. Then $A \mapsto \mathbb{F}^{ \pm}(A)$ is a fully faithful functor from $\mathcal{R}$ ings to $\mathbb{F}^{ \pm}-\mathcal{R}$ ings. All the above examples of $\mathbb{F}^{ \pm}$ rings are $\mathbb{F}^{ \pm, t}$-rings with respect to transposition. Moreover, exterior powers give them the structure of $\mathbb{F}^{\lambda}$-rings.

For a commutative ring $A$ that contains all the roots of unity, together with a fixed map $\mu \rightarrow \mu(A)$ from our abstract group $\mu$ onto the group of roots of unity $\mu(A) \subseteq A^{*}$ (this map could have kernel $\mu_{p^{\infty}}$ if $A$ has characteristic $\left.p\right)$, we have the $\overline{\mathbb{F}}$-ring $\overline{\mathbb{F}}(A)$ with

$$
\overline{\mathbb{F}}(A)_{Y, X}=\operatorname{Hom}_{A}(A \cdot X, A \cdot Y),
$$

where $A \cdot X$ denotes the free $A$-module with basis $X$ divided by the $A$-submodule generated by $\{\zeta \cdot(x)-(\zeta \cdot x) \mid x \in X, \zeta \in \mu\}$. Then $A \mapsto \overline{\mathbb{F}}(A)$ is a fully faithful functor from $\mu$-Rings to $\overline{\mathbb{F}}$ - $\operatorname{Rings}$, where $\mu$-R ings is the category of such commutative rings $A$ together with the map $\mu \rightarrow \mu(A)$, and ring homomorphisms preserving these maps. The $\overline{\mathbb{F}}(A)$ has an involution making it an $\overline{\mathbb{F}}^{\mathrm{t}}$-ring. Moreover, exterior powers give $\overline{\mathbb{F}}(A)$ the structure of $\overline{\mathbb{F}}^{\lambda}$-rings.

Remark. The categories $\mathbb{F}$-R ings (respectively $\mathbb{F}^{ \pm}$-R ings, $\mathbb{F}^{\mathrm{t}}-\mathcal{R}$ ings, $\mathbb{F}^{\lambda}-\mathcal{R}$ ings, $\overline{\mathbb{F}}-\mathcal{R}$ ings, $\overline{\mathbb{F}}^{\mathrm{t}}-\mathcal{R}$ ings, $\overline{\mathbb{F}}^{\lambda}$-R ings) have fibred products. Given homomorphisms of $\mathbb{F}$-rings

$$
\varphi^{i}: A^{i} \rightarrow B, \quad i=0,1,
$$

we have the $\mathbb{F}$-ring $A^{0} \prod_{B} A^{1}$, with

$$
\left(A^{0} \prod_{B} A^{1}\right)_{Y, X}=\left\{\left(a_{0}, a_{1}\right) \in A_{Y, X}^{0} \times A_{Y, X}^{1} \mid \varphi^{0}\left(a_{0}\right)=\varphi^{1}\left(a_{1}\right)\right\} .
$$

Similarly we can construct arbitrary products $\prod_{i} A^{i}$, and arbitrary inverse limits $\lim _{\longleftarrow} A^{i}$, where $i \mapsto A^{i}$ is a functor from a small category to $\mathbb{F}$ - $\mathcal{R}$ ings (respectively $\mathbb{F}^{\mathrm{t}}$ - $\mathcal{R}$ ings, etc.).

Definition 2.19. Let $A$ be an $\mathbb{F}$-ring. An equivalence ideal $\mathcal{E}$ is a collection of subsets

$$
\mathcal{E}=\coprod_{Y, X \in|\mathbb{F}|} \mathcal{E}_{Y, X}
$$

with $\mathcal{E}_{Y, X} \subseteq A_{Y, X} \times A_{Y, X}$, such that

$$
\mathcal{E}_{Y, X} \text { is an equivalence relation on } A_{Y, X} \text {. }
$$




\section{J. SHAi HARAN}

For $\left(a, a^{\prime}\right) \in \mathcal{E}_{Y, X}$, and for $b_{1} \in A_{Y^{\prime}, Y}, b_{2} \in A_{X, X^{\prime \prime}}$,

$$
b_{1} \circ\left(a, a^{\prime}\right) \circ b_{2} \stackrel{\text { def }}{=}\left(b_{1} \circ a \circ b_{2}, b_{1} \circ a^{\prime} \circ b_{2}\right) \in \mathcal{E}_{Y^{\prime}, X^{\prime \prime}} .
$$

For $\left(a_{i}, a_{i}^{\prime}\right) \in \mathcal{E}_{Y_{i}, X_{i}}, i=0,1$,

$$
\left(a_{0}, a_{0}^{\prime}\right) \oplus\left(a_{1}, a_{1}^{\prime}\right) \stackrel{\text { def }}{=}\left(a_{0} \oplus a_{1}, a_{0}^{\prime} \oplus a_{1}^{\prime}\right) \in \mathcal{E}_{Y_{0} \oplus Y_{1}, X_{0} \oplus X_{1}} .
$$

For $\left(a, a^{\prime}\right) \in \mathcal{E}_{Y, X}$, and for $b \in A_{W, Z}$,

$$
b \otimes\left(a, a^{\prime}\right) \stackrel{\text { def }}{=}\left(b \otimes a, b \otimes a^{\prime}\right) \in \mathcal{E}_{W \otimes Y, Z \otimes X} .
$$

Given an equivalence ideal $\mathcal{E}$ of $A$, let

$$
A / \mathcal{E}=\coprod_{Y, X \in|\mathbb{F}|} A_{Y, X} / \mathcal{E}_{Y, X}
$$

and let $\pi: A \rightarrow A / \mathcal{E}$ denote the canonical map which associates with $a \in A_{Y, X}$ its equivalence class $\pi(a) \in A_{Y, X} / \mathcal{E}_{Y, X}$. It follows from (2.19.2) (respectively (2.19.3), (2.19.4)) that we have well-defined operations on $A / \mathcal{E}$,

$$
\begin{gathered}
\pi(f) \circ \pi(g)=\pi(f \circ g) \\
(\text { respectively } \pi(f) \oplus \pi(g)=\pi(f \oplus g), \pi(f) \otimes \pi(g)=\pi(f \otimes g)),
\end{gathered}
$$

making $A / \mathcal{E}$ into an $\mathbb{F}$-ring such that $\pi: A \rightarrow A / \mathcal{E}$ is a homomorphism of $\mathbb{F}$-rings.

Given a homomorphism of $\mathbb{F}$-rings $\varphi: A \rightarrow B$ denote by

$$
\begin{aligned}
& \mathcal{K} \mathcal{E} \mathcal{R}(\varphi)=\coprod_{Y, X \in|\mathbb{F}|} \mathcal{K E}_{\mathcal{R}} \mathcal{R}_{Y, X}(\varphi), \\
& \mathcal{K E \mathcal { R }}_{Y, X}(\varphi)=\left\{\left(a, a^{\prime}\right) \in A_{Y, X} \times A_{Y, X} \mid \varphi(a)=\varphi\left(a^{\prime}\right)\right\} .
\end{aligned}
$$

It is clear that $\mathcal{K} \mathcal{E} \mathcal{R}(\varphi)$ is an equivalence ideal of $A$, and that $\varphi$ induces an injection of $\mathbb{F}$-rings $\bar{\varphi}: A / \mathcal{K E R}(\varphi) \hookrightarrow B$, such that $\varphi=\bar{\varphi} \circ \pi$, i.e.

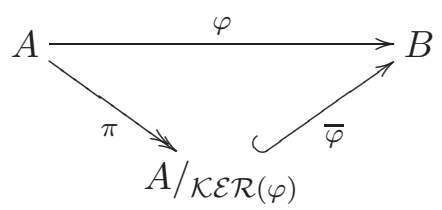

is a commutative diagram. Thus every map $\varphi$ of $\mathbb{F}$-rings factors as an epimorphism $(\pi)$ followed by an injection $(\bar{\varphi})$.

Example 2.22. Let $A=\mathcal{O}_{\mathbb{Z}[1 / N], \eta}$ be the $\mathbb{F}$-ring of Example $4,(2.16)$, with $\mathbb{k}=\mathbb{Z}[1 / N]$. For a prime $\mathfrak{p}$ not dividing $N$ there is a surjective homomorphism

$$
\varphi_{\mathfrak{p}}: A \rightarrow \mathbb{F}\left(\mathbb{F}_{\mathfrak{p}}\right), \quad \varphi_{\mathfrak{p}}(a)=a(\bmod \mathfrak{p}) .
$$

We have the equivalence ideal $\mathcal{E}_{\mathfrak{p}}=\mathcal{K} \mathcal{E} \mathcal{R}\left(\varphi_{\mathfrak{p}}\right)$.

Similarly, there is a surjective homomorphism $\varphi_{\eta}: A \rightarrow \mathbb{F}_{\mathbb{Z}[1 / N], \eta}$, cf. Example 4.21 below, with $\mathbb{F}_{\mathbb{Z}[1 / N], \eta}$ the $\mathbb{F}$-ring of Example $5,(2.18)$, and we have the equivalence ideal $\mathcal{E}_{\eta}=\mathcal{K} \mathcal{E} \mathcal{R}\left(\varphi_{\eta}\right)$.

\section{Modules}

We define the notion of an $A$-module for an $\mathbb{F}$-ring $A$. Since we gave up addition we cannot define directly the quotient $M / N$ where $N$ is a sub- $A$-module of $M$. We can divide $A$-modules only by 


\section{NON-ADDITIVE GEOMETRY}

an equivalence $A$-module, and we study the relationship between sub- $A$-modules and equivalence $A$-modules. We describe the standard operations on $A$-modules and give many examples.

\subsection{Definitions and examples}

Definition 3.1. Let $A$ be an $\mathbb{F}$-ring. An $A$-module $M$ is a collection of sets $M=\left\{M_{Y, X}\right\}_{Y, X \in|\mathbb{F}|}$, together with maps

$$
\begin{aligned}
A_{Y^{\prime}, Y} \times M_{Y, X} \times A_{X, X^{\prime}} \rightarrow M_{Y^{\prime}, X^{\prime}}, \quad\left(a, m, a^{\prime}\right) & \mapsto a \circ m \circ a^{\prime}, \\
A_{Y_{0}, X_{0}} \times M_{Y_{1}, X_{1}} \rightarrow M_{Y_{0} \otimes Y_{1}, X_{0} \otimes X_{1}}, \quad(a, m) & \mapsto a \otimes m, \\
M_{Y_{0}, X_{0}} \times M_{Y_{1}, X_{1}} \rightarrow M_{Y_{0} \oplus Y_{1}, X_{0} \oplus X_{1}}, \quad\left(m_{0}, m_{1}\right) & \mapsto m_{0} \oplus m_{1} .
\end{aligned}
$$

We assume $M_{[0], X}=\left\{0_{X}\right\}, M_{Y,[0]}=\left\{0_{Y}^{\mathrm{t}}\right\}$, and we have a distinguished zero element $0_{Y, X} \in M_{Y, X}$, such that

$$
0 \circ m=0, \quad m \circ 0=0, \quad a \circ 0 \circ a^{\prime}=0, \quad a \otimes 0=0, \quad 0 \oplus 0=0 .
$$

The maps $\circ, \oplus, \otimes$ satisfy: for $a, a^{\prime}, \bar{a}, \bar{a}^{\prime}, a_{i}, a_{i}^{\prime} \in A, m, m_{i} \in M$,

$$
\begin{gathered}
\bar{a} \circ\left(a \circ m \circ a^{\prime}\right) \circ \bar{a}^{\prime}=(\bar{a} \circ a) \circ m \circ\left(a^{\prime} \circ \bar{a}^{\prime}\right), \\
\operatorname{id}_{Y} \circ m \circ \operatorname{id}_{X}=m, \\
\left(a_{0} \oplus a_{1}\right) \circ\left(m_{0} \oplus m_{1}\right) \circ\left(a_{0}^{\prime} \oplus a_{1}^{\prime}\right)=\left(a_{0} \circ m_{0} \circ a_{0}^{\prime}\right) \oplus\left(a_{1} \circ m_{1} \circ a_{1}^{\prime}\right), \\
m_{0} \oplus m_{1}=m_{1} \oplus m_{0}, \\
m_{0} \oplus\left(m_{1} \oplus m_{2}\right)=\left(m_{0} \oplus m_{1}\right) \oplus m_{2}, \\
m \oplus 0_{[0]}=m, \\
(\bar{a} \otimes a) \circ\left(a_{0} \otimes m\right) \circ\left(\bar{a}^{\prime} \otimes a^{\prime}\right)=\left(\bar{a} \circ a_{0} \circ \bar{a}^{\prime}\right) \otimes\left(a \circ m \circ a^{\prime}\right), \\
a_{0} \otimes\left(a_{1} \otimes m\right)=\left(a_{0} \otimes a_{1}\right) \otimes m, \\
\operatorname{id}_{[1]} \otimes m=m, \\
\left(a_{0} \oplus a_{1}\right) \otimes m=\left(a_{0} \otimes m\right) \oplus\left(a_{1} \otimes m\right), \\
a \otimes\left(m_{0} \oplus m_{1}\right)=\left(a \otimes m_{0}\right) \oplus\left(a \otimes m_{1}\right) .
\end{gathered}
$$

In particular, (3.1.2) induces an action of the monoid $A_{[1],[1]}$ on $M_{Y, X}$ via $(a, m) \mapsto a \otimes m$.

Example 3.2.1. Let $A$ be a commutative ring, $\mathbb{F}(A)$ the associated $\mathbb{F}$-ring. For an $A$-module $M$ let $\mathbb{F}(M)_{Y, X}$ denote the $Y \times X$ matrices with values in $M$. Then $\mathbb{F}(M)$ has natural operations (3.1.1), (3.1.2), (3.1.3) making it into an $\mathbb{F}(A)$-module. Note that for $M=A$ we obtain the $\mathbb{F}$-ring $\mathbb{F}(A)$ viewed as an $\mathbb{F}(A)$-module. We have, for $A$-modules $M_{1}, M_{2}$,

$$
\mathbb{F}\left(\operatorname{Hom}_{A}\left(M_{1}, M_{2}\right)\right)_{Y, X}=\operatorname{Hom}_{A}\left(M_{1} \otimes_{A} A \cdot X, M_{2} \otimes_{A} A \cdot Y\right) .
$$

Example 3.2.2. For a finite set $V$ let $\mathbb{F}(V)_{Y, X}$ denote the $Y \times X$ matrices with values in $V \cup\{0\}$ such that every row and every column contains at most one non-zero term. Then $\mathbb{F}(V)$ has natural operations (3.1.1), (3.1.2), (3.1.3) making it into a module over the $\mathbb{F}$-ring $\mathbb{F}$. For $V=[1]$ we obtain $\mathbb{F}([1])$ which is just $\mathbb{F}$ viewed as an $\mathbb{F}$-module. We have, for finite sets $V_{1}, V_{2}$,

$$
\mathbb{F}\left(\operatorname{Hom}_{\mathbb{F}}\left(V_{1}, V_{2}\right)\right)_{Y, X}=\operatorname{Hom}_{\mathbb{F}}\left(V_{1} \otimes X, V_{2} \otimes Y\right) .
$$

For an $\mathbb{F}$-module $W$, such that $W_{Y, X}$ is a finite set for all $X, Y \in|\mathbb{F}|$, we say it has dimension $\operatorname{dim}_{\mathbb{F}} W$ over $\mathbb{F}$ if the following limit exists (where $n, m$ go to infinity independently of each other):

$$
\operatorname{dim}_{\mathbb{F}} W=\lim _{n, m \rightarrow \infty} \frac{1}{n m} \log \sharp W_{[n],[m]} .
$$

Thus if $V$ is a finite dimensional vector space over the finite field $\mathbb{F}_{q}$, and $\mathbb{F}(V)$ the associated 


\section{J. SHAi HARAN}

$\mathbb{F}\left(\mathbb{F}_{q}\right)$-module viewed as $\mathbb{F}$-module, we have

$$
\operatorname{dim}_{\mathbb{F}} \mathbb{F}(V)=\operatorname{dim}_{\mathbb{F}} \mathbb{F}\left(\mathbb{F}_{q}\right) \cdot \operatorname{dim}_{\mathbb{F}_{q}} V
$$

with $\operatorname{dim}_{\mathbb{F}} \mathbb{F}\left(\mathbb{F}_{q}\right)=\log q$.

For a finite set $V$, the associated $\mathbb{F}$-module $\mathbb{F}(V)$ is zero dimensional in the above sense, $\operatorname{dim}_{\mathbb{F}} \mathbb{F}(V)=0$. We can use a different dimension function, $\operatorname{Dim}_{\mathbb{F}} W$ for $W$ an $\mathbb{F}$-module (with $W_{[n],[m]}$ finite for all $\left.n, m\right)$, given by

$$
\operatorname{Dim}_{\mathbb{F}} W=\lim _{x, y \rightarrow \infty} \frac{1}{x y} \log \sum_{n, m \geqslant 0}\left(\sharp W_{[n],[m]}\right) \frac{x^{n}}{n !} \frac{y^{m}}{m !} .
$$

For the $\mathbb{F}$-module $W=\mathbb{F}(V), V$ a finite set, it gives

$$
\begin{aligned}
\operatorname{Dim}_{\mathbb{F}} \mathbb{F}(V) & =\lim _{x, y \rightarrow \infty} \frac{1}{x y} \log \sum_{n, m \geqslant k}\left(\begin{array}{l}
n \\
k
\end{array}\right)\left(\begin{array}{c}
m \\
k
\end{array}\right) k !(\sharp V)^{k} \frac{x^{n}}{n !} \frac{y^{m}}{m !} \\
& =\lim _{x, y \rightarrow \infty} \frac{1}{x y} \log \exp (x+y+x y(\sharp V))=\sharp V .
\end{aligned}
$$

Indeed, to give an arbitrary element of $\mathbb{F}(V)_{[n],[m]}$ we have to choose $k$ rows (respectively, $k$ columns), and there are $\left(\begin{array}{l}n \\ k\end{array}\right)$ (respectively, $\left(\begin{array}{c}m \\ k\end{array}\right)$ ) choices, then we have to choose a bijection between these rows and columns (there are $k$ ! possibilities for such a bijection), and finally we have to fill in the $k$ chosen entries with elements of $V$ (and there are $(\sharp V)^{k}$ such choices), hence

$$
\sharp \mathbb{F}(V)_{[n],[m]}=\sum_{k \leqslant n, m}\left(\begin{array}{l}
n \\
k
\end{array}\right)\left(\begin{array}{c}
m \\
k
\end{array}\right) k !(\sharp V)^{k} .
$$

Example 3.2.3. Let $\mathbb{k}$ be a field, $\eta: \mathbb{k} \rightarrow \mathbb{C}$ an embedding, and let $V$ be a $\mathbb{k}$-vector space with an inner product $(\cdot, \cdot)_{V}$ and associated norm $\|\cdot\|_{V}$. Let $\mathbb{F}(V)_{Y, X}$ denote the $Y \times X$ matrices with values in $V, \mathrm{v}=\left(v_{y, x}\right)$, such that for $\mathrm{a}=\left(a_{x}\right) \in \mathbb{k} \cdot X, \mathrm{~b}=\left(b_{y}\right) \in \mathbb{k} \cdot Y$, we have (cf. (2.16.1))

$$
\left\|\sum_{x, y} b_{y} v_{y, x} a_{x}\right\|_{V} \leqslant|\mathrm{a}|_{\eta} \cdot|\mathrm{b}|_{\eta} .
$$

The set $\mathbb{F}(V)$ has natural operations (3.1.1), (3.1.2), (3.1.3) making it into an $\mathcal{O}_{\mathbb{k}, \eta}$-module. For $V=\mathbb{k}$ we obtain $\mathbb{F}(\mathbb{k})$ which is $\mathcal{O}_{\mathbb{k}, \eta}$ viewed as an $\mathcal{O}_{\mathbb{k}, \eta}$-module.

Definition 3.3. Let $A$ be an $\mathbb{F}$-ring, and $M, M^{\prime}$ be $A$-modules. A collection of maps

$$
\varphi=\left\{\varphi_{Y, X}: M_{Y, X} \rightarrow M_{Y, X}^{\prime}|Y, X \in| \mathbb{F} \mid\right\}
$$

is a homomorphism of $A$-modules if it respects the operations

$$
\begin{gathered}
\varphi\left(a \circ m \circ a^{\prime}\right)=a \circ \varphi(m) \circ a^{\prime}, \\
\varphi(a \otimes m)=a \otimes \varphi(m), \\
\varphi\left(m_{0} \oplus m_{1}\right)=\varphi\left(m_{0}\right) \oplus \varphi\left(m_{1}\right) .
\end{gathered}
$$

The collection of $A$-modules and homomorphisms form a category $A$-Mod. It has an initial and final object $0=\left\{\left\{0_{Y, X}\right\}\right\}_{Y, X \in|\mathbb{F}|}$. For a commutative ring $A$, the construction of Example 3.2.1 gives a functor

$$
\mathbb{F}: A-\mathcal{M o d} \rightarrow \mathbb{F}(A) \text {-Mod, } \quad M \mapsto \mathbb{F}(M) .
$$

As in (2.13.4) we see that this functor is fully faithful.

Similarly, the construction of Example 3.2.2 gives us a functor

$$
\mathbb{F}: \mathbb{F} \rightarrow \mathbb{F}-\mathcal{M o d}, \quad V \mapsto \mathbb{F}(V) .
$$




\section{NON-ADDITIVE GEOMETRY}

For a field embedding $\eta: \mathbb{k} \hookrightarrow \mathbb{C}$, let $(\mathbb{k}, \eta)$-V ec denote the category whose objects are $\mathbb{k}$-vector spaces with an inner product and morphisms are $\mathbb{k}$-linear maps with operator norm at most 1 ; the construction of Example 3.2.3 gives a functor

$$
\mathbb{F}:(\mathbb{k}, \eta)-\mathcal{V} e c \rightarrow \mathcal{O}_{\mathbb{k}, \eta^{-}} \mathcal{M} o d, \quad V \mapsto \mathbb{F}(V) .
$$

\section{$3.2 A$-submodules and equivalence $A$-modules}

Definition 3.5. Let $A$ be an $\mathbb{F}$-ring, $M$ an $A$-module. An $A$-submodule $M^{\prime}$ of $M$ is a collection of subsets $M^{\prime}=\left\{M_{Y, X}^{\prime} \subseteq M_{Y, X}\right\}$ which is closed under the operations $\circ, \oplus, \otimes$ :

$$
A \circ M^{\prime} \circ A \subseteq M^{\prime}, \quad A \otimes M^{\prime} \subseteq M^{\prime}, \quad M^{\prime} \oplus M^{\prime} \subseteq M^{\prime} .
$$

We denote by $\operatorname{sub}_{A}(M)$ the collection of $A$-submodules of $M$. The intersection of $A$-submodules is again an $A$-submodule. An $A$-submodule of $A$ is called an ideal.

Let

$$
\varphi: M \rightarrow N
$$

be a homomorphism of $A$-modules. We have an $A$-submodule of $M$ :

$$
\varphi^{-1}(0)=\{m \in M \mid \varphi(m)=0\} .
$$

It is the kernel of $\varphi$ in the category $A$-Mod.

We have also an $A$-submodule of $N$ :

$$
\varphi(M)=\left\{\varphi_{Y, X}\left(M_{Y, X}\right)\right\}_{Y, X \in|\mathbb{F}|} .
$$

The homomorphism $\varphi$ induces maps

$$
\begin{gathered}
\varphi_{*}: \operatorname{sub}_{A}(M) \rightarrow \operatorname{sub}_{A}(N), \quad M^{\prime} \mapsto \varphi_{*} M^{\prime} \stackrel{\text { def }}{=} \varphi\left(M^{\prime}\right), \\
\varphi^{*}: \operatorname{sub}_{A}(N) \rightarrow \operatorname{sub}_{A}(M), \quad N^{\prime} \mapsto \varphi^{*} N^{\prime} \stackrel{\text { def }}{=} \varphi^{-1}\left(N^{\prime}\right) .
\end{gathered}
$$

The category $A$-Mod has fibred products. Given $A$-Mod homomorphisms

$$
\varphi_{0}: M_{0} \rightarrow M \leftarrow M_{1}: \varphi_{1}
$$

we have the $A$-module

$$
M_{0} \prod_{M} M_{1}
$$

with

$$
\left(M_{0} \prod_{M} M_{1}\right)_{Y, X}=\left\{\left(m_{0}, m_{1}\right), \quad m_{i} \in\left(M_{i}\right)_{Y, X}, \quad \varphi_{0}\left(m_{0}\right)=\varphi_{1}\left(m_{1}\right)\right\}
$$

and the operations

$$
\begin{gathered}
a \circ\left(m_{0}, m_{1}\right) \circ a^{\prime}=\left(a \circ m_{0} \circ a^{\prime}, a \circ m_{1} \circ a^{\prime}\right), \\
a \otimes\left(m_{0}, m_{1}\right)=\left(a \otimes m_{0}, a \otimes m_{1}\right), \\
\left(m_{0}, m_{1}\right) \oplus\left(m_{0}^{\prime}, m_{1}^{\prime}\right)=\left(m_{0} \oplus m_{0}^{\prime}, m_{1} \oplus m_{1}^{\prime}\right) .
\end{gathered}
$$

In particular we have products $M_{0} \prod M_{1}$. We can similarly form arbitrary products $\prod_{\lambda} M_{\lambda}$, and arbitrary inverse limits

$$
\lim _{\longleftarrow} M_{\lambda}=\left\{\left(m_{\lambda}\right) \in \prod M_{\lambda} \mid \varphi_{\lambda^{\prime}, \lambda}\left(m_{\lambda}\right)=m_{\lambda^{\prime}}\right\},
$$

where $\lambda \mapsto M_{\lambda}$ is a functor from a small category to $A$ - $\mathcal{M}$ od. 


\section{J. Shai HARAN}

Let $\varphi: M \rightarrow N$ be a homomorphism of $A$-modules. Let

$$
\mathcal{K} \mathcal{E} \mathcal{R}(\varphi)_{Y, X}=\left\{\left(m, m^{\prime}\right) \in\left(M \prod M\right)_{Y, X} \mid \varphi(m)=\varphi\left(m^{\prime}\right)\right\}=M \prod_{N} M .
$$

Then $\mathcal{K} \mathcal{E R}(\varphi)$ is an $A$-submodule of $M \prod M$ such that, for all $Y, X \in|\mathbb{F}|, \mathcal{K} \mathcal{E} \mathcal{R}(\varphi)_{Y, X}$ is an equivalence relation on $M_{Y, X}$.

Definition 3.9. Let $M$ be an $A$-module. An equivalence $A$-module of $M$ is an $A$-submodule $\mathcal{E}$ of $M \prod M$, such that $\mathcal{E}_{Y, X}$ is an equivalence relation on $M_{Y, X}$. We $\operatorname{denote}$ by equiv ${ }_{A}(M)$ the collection of equivalence $A$-modules of $M$.

For $\mathcal{E} \in \operatorname{equiv}_{A}(M)$ we can form the equivalence classes $(M / \mathcal{E})_{Y, X}=M_{Y, X} / \mathcal{E}_{Y, X}$. There is an induced $A$-module structure on $M / \mathcal{E}$ such that the canonical map $\pi: M \rightarrow M / \mathcal{E}$ is a homomorphism. We have

$$
\operatorname{Hom}_{A-\mathcal{M o d}}(M / \mathcal{E}, N)=\left\{\varphi \in \operatorname{Hom}_{A-\mathcal{M o d}}(M, N) \mid \mathcal{K} \mathcal{E} \mathcal{R}(\varphi) \supseteq \mathcal{E}\right\} .
$$

We have one-to-one order-preserving correspondence

$$
\operatorname{equiv}_{A}(M / \mathcal{E}) \cong\left\{\mathcal{E}^{\prime} \in \operatorname{equiv}_{A}(M) \mid \mathcal{E}^{\prime} \supseteq \mathcal{E}\right\}, \quad \mathcal{E}^{\prime} / \mathcal{E} \mapsto \mathcal{E}^{\prime},
$$

and a natural isomorphism

$$
(M / \mathcal{E}) /\left(\mathcal{E}^{\prime} / \mathcal{E}\right) \cong M / \mathcal{E}^{\prime}
$$

Definition 3.9.4. For an equivalence $A$-module $\mathcal{E}$ of $M$, a submodule $M_{0} \subseteq M$ is called $\mathcal{E}$-stable if for all $\left(m, m^{\prime}\right) \in \mathcal{E}$,

$$
m \in M_{0} \Leftrightarrow m^{\prime} \in M_{0} .
$$

We have a one-to-one order-preserving correspondence

$$
\operatorname{sub}_{A}(M / \mathcal{E}) \cong\left\{M_{0} \in \operatorname{sub}_{A}(M) \mid M_{0} \text { is } \mathcal{E} \text {-stable }\right\}, \quad M_{0} / \mathcal{E} \mapsto M_{0} .
$$

Every homomorphism of $A$-modules $\varphi: M \rightarrow N$ factors as (injection) $\circ$ (surjection), as in the diagram.

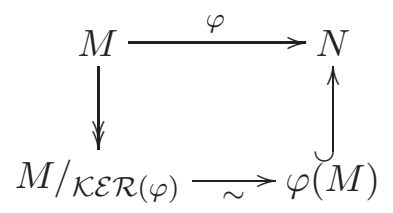

Definition 3.10. For an equivalence $A$-module $\mathcal{E} \subseteq M \prod M$ let

$$
Z(\mathcal{E})=\pi^{-1}(0)=\{m \in M \mid(m, 0) \in \mathcal{E}\}=\mathcal{E} \cap\left(M \prod\{0\}\right) .
$$

It is an $A$-submodule of $M$. For an $A$-submodule $M_{0} \subseteq M$ let

$$
E\left(M_{0}\right) \subseteq M \prod M
$$

be the equivalence $A$-module of $M$ generated by $\left\{(m, 0) \mid m \in M_{0}\right\}$, i.e. $E\left(M_{0}\right)$ is the intersection $\bigcap \mathcal{E}$ of all equivalence $A$-modules $\mathcal{E}$ of $M$ such that $M_{0} \times\{0\} \subseteq \mathcal{E}$. We write $M / M_{0}$ for $M / E\left(M_{0}\right)$. For a homomorphism of $A$-modules $\varphi: M \rightarrow N$ we have its cokernel,

$$
\operatorname{Coker}(\varphi)=N / \varphi(M)=N / E(\varphi(M)) .
$$




\section{NON-ADDITIVE GEOMETRY}

Lemma 3.11. We have

$$
\begin{gathered}
M_{0} \subseteq M_{0}^{\prime} A \text {-submodules of } M \Rightarrow E\left(M_{0}\right) \subseteq E\left(M_{0}^{\prime}\right), \\
\mathcal{E} \subseteq \mathcal{E}^{\prime} \subseteq M \prod M \text { equivalence } A \text {-modules of } M \Rightarrow Z(\mathcal{E}) \subseteq Z\left(\mathcal{E}^{\prime}\right), \\
M_{0} \subseteq Z\left(E\left(M_{0}\right)\right), \\
E(Z(\mathcal{E})) \subseteq \mathcal{E} .
\end{gathered}
$$

Proof. The proof is straightforward.

Corollary 3.12. We have

$$
\begin{aligned}
E\left(M_{0}\right) & =E\left(Z\left(E\left(M_{0}\right)\right)\right), \\
Z(\mathcal{E}) & =Z(E(Z(\mathcal{E}))) .
\end{aligned}
$$

Hence we have

$$
\left\{Z(\mathcal{E}) \mid \mathcal{E} \in \operatorname{equiv}_{A}(M)\right\}=\left\{M_{0} \subseteq M \mid M_{0}=Z\left(E\left(M_{0}\right)\right)\right\} ;
$$

we denote this set by $E$-sub $\operatorname{su}_{A}(M)$.

Similarly, we have

$$
\left\{E\left(M_{0}\right) \mid M_{0} \subseteq M A \text {-submodule }\right\}=\left\{\mathcal{E} \subseteq M \prod M \mid \mathcal{E}=E(Z(\mathcal{E}))\right\} ;
$$

we denote this set by $Z$-equiv $_{A}(M)$.

Moreover, there is an induced bijection

$$
\begin{gathered}
E-\operatorname{sub}_{A}(M) \stackrel{\sim}{\leftrightarrow} Z-\operatorname{equiv}_{A}(M), \\
M_{0} \mapsto E\left(M_{0}\right), \\
Z(\mathcal{E}) \longleftrightarrow \mathcal{E} .
\end{gathered}
$$

Lemma 3.13. Let $M_{0} \subseteq M$ be an $A$-submodule, and let $\mathcal{E}_{Y, X} \subseteq\left(M \prod M\right)_{Y, X}$ denote the collection of pairs $\left(m, m^{\prime}\right)$ such that there exists a 'path' $m=m_{0}, m_{1}, \ldots, m_{l}=m^{\prime}$, where for $j=0, \ldots, l-1$, $\left\{m_{j}, m_{j+1}\right\}$ has the form $\left\{a \circ\left(n \oplus n_{0}\right) \circ a^{\prime}, a \circ(n \oplus 0) \circ a^{\prime}\right\}$ for some $a, a^{\prime} \in A, n \in M, n_{0} \in M_{0}$. Then $E\left(M_{0}\right)=\mathcal{E}$.

Proof. Note that for $a, a^{\prime} \in A, n \in M, n_{0} \in M_{0}$, we have

$$
\left(m_{0}, 0\right) \in E\left(M_{0}\right),(m, m) \in E\left(M_{0}\right)
$$

and since $E\left(M_{0}\right) \subseteq M \prod M$ is a submodule we get

$$
\left(a \circ\left(n \oplus n_{0}\right) \circ a^{\prime}, a \circ(n \oplus 0) \circ a^{\prime}\right) \in E\left(M_{0}\right) .
$$

Thus if there is a path $m=m_{0}, \ldots, m_{l}=m^{\prime}$ as above, then $\left(m, m^{\prime}\right) \in E\left(M_{0}\right)$; so $\mathcal{E} \subseteq E\left(M_{0}\right)$.

For the reverse inclusion note that $\mathcal{E}_{Y, X}$ is an equivalence relation on $M_{Y, X}$. Moreover, $\mathcal{E}$ is an $A$-submodule of $M \prod M$. For $\left(m, m^{\prime}\right) \in \mathcal{E}$ there exists a path $m=m_{0}, \ldots, m_{l}=m^{\prime}$ as above, hence for $a, a^{\prime} \in A$,

$$
a \circ m_{0} \circ a^{\prime}, \ldots, a \circ m_{l} \circ a^{\prime}
$$

is a path from $a \circ m \circ a^{\prime}$ to $a \circ m^{\prime} \circ a^{\prime}$, hence

$$
\left(a \circ m \circ a^{\prime}, a \circ m^{\prime} \circ a^{\prime}\right) \in \mathcal{E} .
$$

Similarly, for $\bar{a} \in A_{Y, X}$,

$$
\bar{a} \otimes m_{0}, \ldots, \bar{a} \otimes m_{l}
$$




\section{J. Shai HARAN}

is a path, which shows that

$$
\left(\bar{a} \otimes m, \bar{a} \otimes m^{\prime}\right) \in \mathcal{E} .
$$

To show this use

$$
\begin{gathered}
\bar{a} \otimes\left(a \circ\left(n \oplus n_{0}\right) \circ a^{\prime}\right)=(\bar{a} \otimes a) \circ\left(\left(\operatorname{id}_{X} \otimes n\right) \oplus\left(\operatorname{id}_{X} \otimes n_{0}\right)\right) \circ\left(\operatorname{id}_{X} \otimes a^{\prime}\right), \\
\operatorname{id}_{X} \otimes n_{0} \in M_{0}, \quad \operatorname{id}_{X} \otimes 0=0 .
\end{gathered}
$$

If also $\left(\bar{m}, \bar{m}^{\prime}\right) \in \mathcal{E}$, we can assume the path $\bar{m}=\overline{m_{0}}, \ldots, \overline{m_{l}}=\bar{m}^{\prime}$ has the same length $l$ (by adding identities $n_{0}=0, a=\mathrm{id}, a^{\prime}=\mathrm{id}$ ), and then

$$
m_{0} \oplus \overline{m_{0}}, \ldots, m_{l} \oplus \overline{m_{l}}
$$

is a path, which shows that

$$
\left(m \oplus \bar{m}, m^{\prime} \oplus \bar{m}^{\prime}\right) \in \mathcal{E} .
$$

To show this use

$$
\left(a \circ\left(n \oplus n_{0}\right) \circ a^{\prime}\right) \oplus\left(\bar{a} \circ\left(\bar{n} \oplus \overline{n_{0}}\right) \circ \bar{a}^{\prime}\right)=(a \oplus \bar{a}) \circ\left((n \oplus \bar{n}) \oplus\left(n_{0} \oplus \overline{n_{0}}\right)\right) \circ\left(a^{\prime} \oplus \bar{a}^{\prime}\right) .
$$

Thus $\mathcal{E}$ is an equivalence $A$-submodule of $M$, and since $\left\{\left(m_{0}, 0\right) \mid m_{0} \in M_{0}\right\} \subseteq \mathcal{E}$, we have $E\left(M_{0}\right) \subseteq \mathcal{E}$.

Corollary 3.14. Let $M_{0} \subseteq M$ be an A-submodule. We have

$$
Z\left(E\left(M_{0}\right)\right)=M_{0}
$$

if and only if, for all $m_{0} \in M_{0}, m \in M, a, a^{\prime} \in A$,

$$
a \circ\left(m \oplus m_{0}\right) \circ a^{\prime} \in M_{0} \quad \Leftrightarrow \quad a \circ(m \oplus 0) \circ a^{\prime} \in M_{0},
$$

i.e. $M_{0} \in E-\operatorname{sub}_{A}(M)$ if and only if $M_{0}$ is $E\left(M_{0}\right)$-stable.

Proof. Assume (3.14.1) holds. By Lemma 3.13 if $\left(m, m^{\prime}\right) \in E\left(M_{0}\right)$ there exists a path $m=$ $m_{0}, \ldots, m_{l}=m^{\prime}$, and we have

hence

$$
m_{j} \in M_{0} \quad \Leftrightarrow \quad m_{j+1} \in M_{0},
$$

$$
m \in M_{0} \quad \Leftrightarrow \quad m^{\prime} \in M_{0} .
$$

Taking $m^{\prime}=0 \in M_{0}$, we get $(m, 0) \in E\left(M_{0}\right)$ implies $m \in M_{0}$. Thus $Z\left(E\left(M_{0}\right)\right) \subseteq M_{0}$, and since the reverse inclusion always holds we get $Z\left(E\left(M_{0}\right)\right)=M_{0}$.

Conversely, assume $Z\left(E\left(M_{0}\right)\right)=M_{0}$, then

$$
\begin{aligned}
a \circ\left(m \oplus m_{0}\right) \circ a^{\prime} \in M_{0} & \Leftrightarrow \quad\left(a \circ\left(m \oplus m_{0}\right) \circ a^{\prime}, 0\right) \in E\left(M_{0}\right), \\
a \circ(m \oplus 0) \circ a^{\prime} \in M_{0} & \Leftrightarrow \quad\left(a \circ(m \oplus 0) \circ a^{\prime}, 0\right) \in E\left(M_{0}\right) .
\end{aligned}
$$

Using the fact that $E\left(M_{0}\right)_{Y, X}$ is an equivalence relation, and that for $m_{0} \in M_{0}$

$$
\left(a \circ\left(m \oplus m_{0}\right) \circ a^{\prime}, a \circ(m \oplus 0) \circ a^{\prime}\right) \in E\left(M_{0}\right),
$$

we see that the statements in (3.14.2) and (3.14.3) are equivalent, hence (3.14.1) holds.

For submodules $M_{0} \in \operatorname{sub}_{A}(M), M^{\prime} \in E-\operatorname{sub}_{A}(M)$, we have $M^{\prime} \supseteq M_{0}$ if and only if $M^{\prime}$ is $E\left(M_{0}\right)$-stable. We get a one-to-one order-preserving correspondence

$$
E-\operatorname{sub}_{A}\left(M / M_{0}\right)=\left\{M^{\prime} \in E-\operatorname{sub}_{A}(M) \mid M^{\prime} \supseteq M_{0}\right\}, \quad M^{\prime} / M_{0} \mapsto M^{\prime}
$$

and a natural isomorphism

$$
\left(M / M_{0}\right) /\left(M^{\prime} / M_{0}\right) \cong M / M^{\prime}
$$




\section{NON-ADDITIVE GEOMETRY}

An $A$-submodule of $A$ is called an ideal, and an equivalence $A$-module of $A$ is called an equivalence ideal. Thus we have the maps $E, Z$ between ideals and equivalence ideals satisfying Lemma 3.11 and Corollary 3.12. Elements of $E$-sub $A(A)$ will be called $E$-ideals.

Example 3.15.3. For $A=\mathcal{O}_{\mathbb{Z}[1 / N], \eta}$, with the notation of Example 2.22, we have for $\mathfrak{p} \nmid N: E Z\left(\mathcal{E}_{\mathfrak{p}}\right) \varsubsetneqq$ $\mathcal{E}_{\mathfrak{p}} \cap \mathcal{E}_{\eta}$.

\subsection{Operations on submodules}

For a family $\left\{M_{i}\right\}$ of $A$-submodules of $M$, we have the intersection $\bigcap_{i} M_{i} \in \operatorname{sub}_{A}(M)$. Note that if $M_{i} \in E-\operatorname{sub}_{A}(M)$ then $\bigcap_{i} M_{i} \in E$-sub $A(M)$. We have also $\sum_{i} M_{i}$ the $A$-submodule generated by the $M_{i}$, i.e. it is the intersection $\bigcap N$ taken over all submodules $N$ that contain all the $M_{i}$. It can be described explicitly as

$$
\left(\sum_{i} M_{i}\right)_{Y, X}=\left\{a \circ\left(\bigoplus_{i} m_{i}\right) \circ a^{\prime} \mid a \in A_{Y, \oplus_{i} Y_{i}}, a^{\prime} \in A_{\oplus_{i} X_{i}, X}, m_{i} \in\left(M_{i}\right)_{Y_{i}, X_{i}}\right\} .
$$

Indeed the right-hand side will be contained in any submodule $N$ which contains all the $M_{i}$, it itself contains the $M_{i}$, and is closed under the module operations

$$
\begin{gathered}
b \circ\left(a \circ\left(\bigoplus_{i} m_{i}\right) \circ a^{\prime}\right) \circ b^{\prime}=(b \circ a) \circ\left(\bigoplus_{i} m_{i}\right) \circ\left(a^{\prime} \circ b^{\prime}\right), \\
\left(a \circ\left(\bigoplus_{i} m_{i}\right) \circ a^{\prime}\right) \oplus\left(b \circ\left(\bigoplus_{i} m_{i}^{\prime}\right) \circ b^{\prime}\right)=(a \oplus b) \circ\left(\bigoplus_{i}\left(m_{i} \oplus m_{i}^{\prime}\right)\right) \circ\left(a^{\prime} \oplus b^{\prime}\right), \\
b \otimes\left(a \circ\left(\bigoplus_{i} m_{i}\right) \circ a^{\prime}\right)=\left(\operatorname{id}_{Y} \otimes a\right) \circ\left(\bigoplus_{i}\left(b \otimes m_{i}\right)\right) \circ\left(\operatorname{id}_{X} \otimes a^{\prime}\right), b \in A_{Y, X} .
\end{gathered}
$$

More generally, given any subset $\left\{m_{i} \mid i \in I\right\} \subseteq M$, with $m_{i} \in M_{Z_{i}, W_{i}}$, the $A$-submodule it generates $\sum_{i} A \cdot m_{i}$ can be described explicitly as

$$
\left(\sum_{i} A \cdot m_{i}\right)_{Y, X}=\left\{a \circ\left(\bigoplus_{i} \operatorname{id}_{X_{i}} \otimes m_{i}\right) \circ a^{\prime} \mid a \in A_{Y, \oplus_{i}\left(X_{i} \otimes Z_{i}\right)}, a^{\prime} \in A_{\oplus_{i}\left(X_{i} \otimes W_{i}\right), X}\right\} .
$$

Given an $A$-module $M$ and an ideal $\mathfrak{a} \subseteq A$ we have their product $\mathfrak{a} \cdot M$ which is an $A$-submodule of $M$,

$$
\begin{aligned}
& (\mathfrak{a} \cdot M)_{Y, X} \\
& \quad=\left\{b \circ\left(\bigoplus_{i}\left(a_{i} \otimes m_{i}\right)\right) \circ b^{\prime} \mid b \in A_{Y, \oplus_{i}\left(Y_{i} \otimes Z_{i}\right)}, b^{\prime} \in A_{\bigoplus_{i}\left(X_{i} \otimes W_{i}\right), X}, a_{i} \in \mathfrak{a}_{Y_{i}, X_{i}}, m_{i} \in M_{Z_{i}, W_{i}}\right\} .
\end{aligned}
$$

Given $A$-submodules $M_{0}, M_{1}$ of $M$ we can form their quotient

$$
\left(M_{0}: M_{1}\right)=\left\{a \in A \mid a \otimes m \in M_{0} \forall m \in M_{1}\right\} .
$$

It is easily checked that $\left(M_{0}: M_{1}\right)$ is an ideal of $A$.

\subsection{Operations on modules}

Sums. Given $A$-modules $M_{0}, M_{1}$, we first construct the sum (coproduct) $M_{0} \amalg M_{1}$ in the category $A$-Mod. We form

$$
\left(M_{0} \coprod M_{1}\right)_{Y, X}=\left\{\left(a, m_{0}, m_{1}, a^{\prime}\right) \mid a \in A_{Y, Y_{0} \oplus Y_{1}}, a^{\prime} \in A_{X_{0} \oplus X_{1}, X}, m_{i} \in\left(M_{i}\right)_{Y_{i}, X_{i}}\right\} / \sim
$$




\section{J. SHAi HARAN}

where $\sim$ is the equivalence relation generated by

$$
\begin{aligned}
& \left(a \circ\left(a_{0} \oplus a_{1}\right), m_{0}, m_{1}, a^{\prime}\right) \sim\left(a, a_{0} \circ m_{0}, a_{1} \circ m_{1}, a^{\prime}\right), \\
& \left(a, m_{0}, m_{1},\left(a_{0}^{\prime} \oplus a_{1}^{\prime}\right) \circ a^{\prime}\right) \sim\left(a, m_{0} \circ a_{0}^{\prime}, m_{1} \circ a_{1}^{\prime}, a^{\prime}\right) .
\end{aligned}
$$

Let $\left(a, m_{0}, m_{1}, a^{\prime}\right) / \sim$ denote the equivalence class containing $\left(a, m_{0}, m_{1}, a^{\prime}\right)$. Define

$$
\begin{gathered}
b \circ\left(a, m_{0}, m_{1}, a^{\prime}\right) / \sim \circ b^{\prime}=\left(b \circ a, m_{0}, m_{1}, a^{\prime} \circ b^{\prime}\right) / \sim, \\
\left(a, m_{0}, m_{1}, a^{\prime}\right) / \sim \oplus\left(\bar{a}, \bar{m}_{0}, \bar{m}_{1}, \bar{a}^{\prime}\right) / \sim=\left(a \oplus \bar{a}, m_{0} \oplus \bar{m}_{0}, m_{1} \oplus \bar{m}_{1}, a^{\prime} \oplus \bar{a}^{\prime}\right) / \sim, \\
b \otimes\left(a, m_{0}, m_{1}, a^{\prime}\right) / \sim=\left(b \otimes a, \operatorname{id}_{Z} \otimes m_{0}, \operatorname{id}_{Z} \otimes m_{1}, \operatorname{id}_{Z} \otimes a^{\prime}\right) / \sim .
\end{gathered}
$$

Proposition 3.17. The operations (3.17.3), (3.17.4), (3.17.5) are well defined, independent of the chosen representatives, and make $M_{0} \amalg M_{1}$ into an $A$-module. There are canonical homomorphisms $M_{i} \rightarrow M_{0} \amalg M_{1}$, taking $m$ in $\left(M_{0}\right)_{Y, X}$ (respectively, $\left.\left(M_{1}\right)_{Y, X}\right)$ into $\left(\operatorname{id}_{Y}, m, 0, \operatorname{id}_{X}\right) / \sim($ respectively, $\left.\left(\operatorname{id}_{Y}, 0, m, \operatorname{id}_{X}\right) / \sim\right)$. These maps make $M_{0} \coprod M_{1}$ into the sum of $M_{0}, M_{1}$ in the category $A$-Mod,

$$
\begin{array}{r}
\operatorname{Hom}_{A-\mathcal{M} o d}\left(M_{0}, N\right) \times \operatorname{Hom}_{A-\mathcal{M o d}}\left(M_{1}, N\right)=\operatorname{Hom}_{A-\mathcal{M} \text { od }}\left(M_{0} \coprod M_{1}, N\right), \\
\left(\varphi_{0}, \varphi_{1}\right) \mapsto \varphi_{0} \amalg \varphi_{1}\left(\left(a, m_{0}, m_{1}, a^{\prime}\right) / \sim\right)=a \circ\left(\varphi_{0}\left(m_{0}\right) \oplus \varphi_{1}\left(m_{1}\right)\right) \circ a^{\prime} .
\end{array}
$$

Proof. To show that the operations are well defined we need to show that on replacing the representatives by equivalent ones we get the same result. Since two representatives are equivalent if and only if there is a path between them whose consecutive members are related by (3.17.1) or (3.17.2), it is enough to show that we get the same result for representatives related by (3.17.1), (3.17.2). That $\circ$ in (3.17.3) is well defined follows from associativity of $\circ$. That $\oplus$ is well defined with respect to $(3.17 .1)$ follows from

$$
\begin{aligned}
& \quad\left(\left(a \circ\left(a_{0} \oplus a_{1}\right)\right) \oplus \bar{a}, m_{0} \oplus \bar{m}_{0}, m_{1} \oplus \bar{m}_{1}, a^{\prime} \oplus \bar{a}^{\prime}\right) / \sim \\
& \quad=\left((a \oplus \bar{a}) \circ\left(a_{0} \oplus a_{1} \oplus \mathrm{id}_{\bar{Y}_{0} \oplus \bar{Y}_{1}}\right), m_{0} \oplus \bar{m}_{0}, m_{1} \oplus \bar{m}_{1}, a^{\prime} \oplus \bar{a}^{\prime}\right) / \sim \\
& \quad=\left(a \oplus \bar{a},\left(a_{0} \circ m_{0}\right) \oplus \bar{m}_{0},\left(a_{1} \circ m_{1}\right) \oplus \bar{m}_{1}, a^{\prime} \oplus \bar{a}^{\prime}\right) / \sim .
\end{aligned}
$$

Similarly $\otimes$ in $(3.17 .5)$ is well defined with respect to (3.17.1):

$$
\begin{aligned}
(b & \left.\otimes\left(a \circ\left(a_{0} \oplus a_{1}\right)\right), \mathrm{id}_{Z} \otimes m_{0}, \mathrm{id}_{Z} \otimes m_{1}, \mathrm{id}_{Z} \otimes a^{\prime}\right) / \sim \\
& =\left((b \otimes a) \circ\left(\operatorname{id}_{Z} \otimes a_{0} \oplus \mathrm{id}_{Z} \otimes a_{1}\right), \mathrm{id}_{Z} \otimes m_{0}, \mathrm{id}_{Z} \otimes m_{1}, \mathrm{id}_{Z} \otimes a^{\prime}\right) / \sim \\
& =\left(b \otimes a, \mathrm{id}_{Z} \otimes\left(a_{0} \circ m_{0}\right), \mathrm{id}_{Z} \otimes\left(a_{1} \circ m_{1}\right), \mathrm{id}_{Z} \otimes a^{\prime}\right) / \sim .
\end{aligned}
$$

It is clear that $\circ$ as defined in (3.17.3) is associative (3.1.5) and unitary (3.1.6). It is clear that $\oplus$ as defined in (3.17.4) is functorial (3.1.7), commutative (3.1.8), associative (3.1.9), and unitary (3.1.10). It is clear that $\otimes$ as defined in (3.17.5) is associative (3.1.12), unitary (3.1.13), and distributive (3.1.14), (3.1.15). We check that $\otimes$ is functorial (3.1.11): on the 'left'

$$
\begin{aligned}
(d & \otimes c) \circ\left(b \otimes\left(a, m_{0}, m_{1}, a^{\prime}\right) / \sim\right) \\
& =\left((d \otimes c) \circ(b \otimes a), \operatorname{id}_{Z} \otimes m_{0}, \operatorname{id}_{Z} \otimes m_{1}, \operatorname{id}_{Z} \otimes a^{\prime}\right) / \sim \\
& =\left((d \circ b) \otimes(c \circ a), \operatorname{id}_{Z} \otimes m_{0}, \operatorname{id}_{Z} \otimes m_{1}, \operatorname{id}_{Z} \otimes a^{\prime}\right) / \sim \\
& =(d \circ b) \otimes\left(c \circ\left(a, m_{0}, m_{1}, a^{\prime}\right) / \sim\right) ;
\end{aligned}
$$




\section{NON-ADDITIVE GEOMETRY}

and on the 'right' for $b \in A_{W, Z}, d \in A_{Z, T}$ :

$$
\begin{aligned}
(b & \left.\otimes\left(a, m_{0}, m_{1}, a^{\prime}\right) / \sim\right) \circ(d \otimes c) \\
& =\left(b \otimes a, \mathrm{id}_{Z} \otimes m_{0}, \mathrm{id}_{Z} \otimes m_{1},\left(\mathrm{id}_{Z} \otimes a^{\prime}\right) \circ(d \otimes c)\right) / \sim \\
& =\left(b \otimes a, \mathrm{id}_{Z} \otimes m_{0}, \mathrm{id}_{Z} \otimes m_{1},\left(d \otimes \mathrm{id}_{X_{0} \oplus X_{1}}\right) \circ\left(\mathrm{id}_{T} \otimes\left(a^{\prime} \circ c\right)\right)\right) / \sim \\
& =\left(b \otimes a, d \otimes m_{0}, d \otimes m_{1}, \mathrm{id}_{T} \otimes\left(a^{\prime} \circ c\right)\right) / \sim \\
& =\left((b \otimes a) \circ\left(d \otimes \mathrm{id}_{Y_{0} \oplus Y_{1}}\right), \mathrm{id}_{T} \otimes m_{0}, \mathrm{id}_{T} \otimes m_{1}, \mathrm{id}_{T} \otimes\left(a^{\prime} \circ c\right)\right) / \sim \\
& =\left((b \circ d) \otimes a, \mathrm{id}_{T} \otimes m_{0}, \mathrm{id}_{T} \otimes m_{1}, \mathrm{id}_{T} \otimes\left(a^{\prime} \circ c\right)\right) / \sim \\
& =(b \circ d) \otimes\left(\left(a, m_{0}, m_{1}, a^{\prime}\right) / \sim \circ c\right) .
\end{aligned}
$$

Thus $M_{0} \coprod M_{1}$ is an $A$-module, and it is easy to check that it is the sum of $M_{0}, M_{1}$ in $A$-Mod.

We shall write $a \circ\left(m_{0} \oplus m_{1}\right) \circ a^{\prime}$ for $\left(a, m_{0}, m_{1}, a^{\prime}\right) / \sim$. The canonical map from the sum to the product is given by

$$
\begin{gathered}
M_{0} \coprod M_{1} \rightarrow M_{0} \prod M_{1}, \\
a \circ\left(m_{0} \oplus m_{1}\right) \circ a^{\prime} \mapsto\left(a \circ\left(m_{0} \oplus 0\right) \circ a^{\prime}, a \circ\left(0 \oplus m_{1}\right) \circ a^{\prime}\right) .
\end{gathered}
$$

Given an arbitrary family of $A$-modules $\left\{M_{i}\right\}_{i \in I}$ we can similarly form their sum

$$
\coprod_{i} M_{i}
$$

Direct limits. Given homomorphisms of $A$-modules $\psi_{i}: M \rightarrow M_{i}, i=0,1$, let $\mathcal{E}$ be the equivalence $A$-module of $M_{0} \coprod M_{1}$ generated by

$$
\left\{\left(a \circ\left(\left(m_{0} \oplus \psi_{0}(m)\right) \oplus m_{1}\right) \circ a^{\prime}, a \circ\left(m_{0} \oplus\left(\psi_{1}(m) \oplus m_{1}\right)\right) \circ a^{\prime}\right) \mid m_{i} \in M_{i}, m \in M\right\} .
$$

The quotient

$$
M_{0} \coprod M_{1} / \mathcal{E} \stackrel{\text { def }}{=} M_{0} \coprod_{M} M_{1}
$$

is the push-out

$$
\operatorname{Hom}_{A-\mathcal{M o d}}\left(M_{0} \coprod_{M} M_{1}, N\right)=\left\{\left(\varphi_{0}, \varphi_{1}\right) \mid \varphi_{i} \in \operatorname{Hom}_{A-\mathcal{M} \text { od }}\left(M_{i}, N\right), \varphi_{0} \circ \psi_{0}=\varphi_{1} \circ \psi_{1}\right\} .
$$

Similarly, given a functor $\lambda \mapsto M_{\lambda}$ from a small category to $A$ - $\mathcal{M}$ od, we can form the direct limit

$$
\lim _{\longrightarrow} M_{\lambda}=\coprod_{\lambda} M_{\lambda} / \mathcal{E}
$$

Here $\mathcal{E}$ is the equivalence $A$-module of $\coprod M_{\lambda}$ generated by

$$
\left(\operatorname{id}_{Y} \circ(m \oplus 0 \ldots) \circ \operatorname{id}_{X}, \operatorname{id}_{Y} \circ\left(\varphi_{\lambda^{\prime}, \lambda}(m) \oplus 0 \ldots\right) \circ \operatorname{id}_{X}\right),
$$

where $\varphi_{\lambda^{\prime}, \lambda}: M_{\lambda} \rightarrow M_{\lambda^{\prime}}$ runs over the maps in the direct system, and $m \in M_{\lambda}$.

Free modules. Fix sets $Y_{0}, X_{0} \in|\mathbb{F}|$. Let us form

$$
M_{Y, X}=\left\{\left(a, a^{\prime}\right) \mid a \in A_{Y, Z \otimes Y_{0}}, a^{\prime} \in A_{Z \otimes X_{0}, X}\right\} / \sim,
$$

where $\sim$ is the equivalence relation generated by

$$
\left(a \circ\left(c \otimes \operatorname{id}_{Y_{0}}\right), a^{\prime}\right) \sim\left(a,\left(c \otimes \operatorname{id}_{X_{0}}\right) \circ a^{\prime}\right), \quad a \in A_{Y, Z \otimes Y_{0}}, c \in A_{Z, Z^{\prime}}, a^{\prime} \in A_{Z^{\prime} \otimes X_{0}, X} .
$$




\section{J. Shai Haran}

Let $\left(a, a^{\prime}\right) / \sim$ denote the equivalence class of $\left(a, a^{\prime}\right)$, and define

$$
\begin{gathered}
b \circ\left(a, a^{\prime}\right) / \sim \circ b^{\prime}=\left(b \circ a, a^{\prime} \circ b^{\prime}\right) / \sim, \\
\left(a, a^{\prime}\right) / \sim \oplus\left(\bar{a}, \bar{a}^{\prime}\right) / \sim=\left(a \oplus \bar{a}, a^{\prime} \oplus \bar{a}^{\prime}\right) / \sim, \\
b \otimes\left(a, a^{\prime}\right) / \sim=\left(b \otimes a, \operatorname{id}_{Z} \otimes a^{\prime}\right), b \in A_{W, Z} .
\end{gathered}
$$

Proposition 3.18. The operations (3.18.2), (3.18.3), (3.18.4) are well defined, independent of the representatives, and make $M$ into an $A$-module. It is the 'free' $A$-module in degree $Y_{0}, X_{0}$ :

$$
\operatorname{Hom}_{A-\mathcal{M o d}}(M, N)=N_{Y_{0}, X_{0}} \text { for all } A \text {-modules } N \text {. }
$$

Proof. The proof is similar to that for Proposition 3.17. It follows that $\circ$ is well defined by associativity. That $\oplus$ is well defined follows from

$$
\begin{aligned}
& \left.\left((a)\left(c \otimes \operatorname{id}_{Y_{0}}\right)\right) \oplus \bar{a}, a^{\prime} \oplus \bar{a}^{\prime}\right) / \sim \\
& \quad=\left((a \oplus \bar{a}) \circ\left(\left(c \oplus \mathrm{id}_{\bar{Z}}\right) \otimes \operatorname{id}_{Y_{0}}\right), a^{\prime} \oplus \bar{a}^{\prime}\right) / \sim \\
& \quad=\left(a \oplus \bar{a},\left(\left(c \oplus \mathrm{id}_{\bar{Z}}\right) \otimes \operatorname{id}_{X_{0}}\right) \circ\left(a^{\prime} \oplus \bar{a}^{\prime}\right)\right) / \sim \\
& \quad=\left(a \oplus \bar{a},\left(\left(c \otimes \operatorname{id}_{X_{0}}\right) \circ a^{\prime}\right) \oplus \bar{a}^{\prime}\right) / \sim .
\end{aligned}
$$

Similarly $\otimes$ is well defined:

$$
\begin{aligned}
(b & \left.\otimes\left(a \circ\left(c \otimes \mathrm{id}_{Y_{0}}\right)\right), \mathrm{id}_{W} \otimes a^{\prime}\right) / \sim \\
& =\left((b \otimes a) \circ\left(\mathrm{id}_{W} \otimes c \otimes \mathrm{id}_{Y_{0}}\right), \mathrm{id}_{W} \otimes a^{\prime}\right) / \sim \\
& =\left(b \otimes a,\left(\operatorname{id}_{W} \otimes c \otimes \mathrm{id}_{X_{0}}\right) \circ\left(\operatorname{id}_{W} \otimes a^{\prime}\right)\right) / \sim \\
& =\left(b \otimes a, \mathrm{id}_{W} \otimes\left(\left(c \otimes \mathrm{id}_{X_{0}}\right) \circ a^{\prime}\right)\right) / \sim .
\end{aligned}
$$

It then follows that $M$ satisfies the axioms for $A$-modules; for the functoriality of $\otimes,(3.1 .11)$, we have 'on the left':

$$
\begin{aligned}
(d & \otimes c) \circ\left(b \otimes\left(a, a^{\prime}\right) / \sim\right) \\
& =\left((d \otimes c) \circ(b \otimes a), \operatorname{id}_{Z} \otimes a^{\prime}\right) / \sim \\
& =\left((d \circ b) \otimes(c \circ a), \operatorname{id}_{Z} \otimes a^{\prime}\right) / \sim \\
& =(d \circ b) \otimes\left(c \circ\left(a, a^{\prime}\right) / \sim\right) ;
\end{aligned}
$$

and 'on the right' for $b \in A_{W, Z}, d \in A_{Z, T}$ :

$$
\begin{aligned}
(b & \left.\otimes\left(a, a^{\prime}\right) / \sim\right) \circ(d \otimes c) \\
& =\left(b \otimes a,\left(\mathrm{id}_{Z} \otimes a^{\prime}\right) \circ(d \otimes c)\right) / \sim \\
& =\left(b \otimes a,(d \otimes \mathrm{id}) \circ\left(\operatorname{id}_{T} \otimes\left(a^{\prime} \circ c\right)\right)\right) / \sim \\
& =\left((b \otimes a) \circ(d \otimes \mathrm{id}), \operatorname{id}_{T} \otimes\left(a^{\prime} \circ c\right)\right) / \sim \\
& =\left((b \circ d) \otimes a, \mathrm{id}_{T} \otimes\left(a^{\prime} \circ c\right)\right) / \sim \\
& =(b \circ d) \otimes\left(\left(a, a^{\prime}\right) / \sim c\right) .
\end{aligned}
$$

Thus $M$ is an $A$-module. Given a homomorphism $\varphi: M \rightarrow N$, we get

$$
\varphi\left(\left(\operatorname{id}_{Y_{0}}, \operatorname{id}_{X_{0}}\right)\right) \in N_{Y_{0}, X_{0}} .
$$

Given $n \in N_{Y_{0}, X_{0}}$ we get homomorphism $\varphi$ by

$$
\varphi\left(\left(a, a^{\prime}\right) / \sim\right)=a \circ\left(\operatorname{id}_{Z} \otimes n\right) \circ a^{\prime}, \quad a \in A_{Y, Z \otimes Y_{0}}, a^{\prime} \in A_{Z \otimes X_{0}, X} .
$$

These are inverse to one another and give the bijection (3.18.5).

Let $f=f_{Y_{0}, X_{0}}=\left(\operatorname{id}_{Y_{0}}, \operatorname{id}_{X_{0}}\right) / \sim$. We have $\left(a, a^{\prime}\right) / \sim=a \circ\left(\operatorname{id}_{Z} \otimes f\right) \circ a^{\prime}$. We write $A \cdot f_{Y_{0}, X_{0}}$ for the above module $M$ constructed in (3.18.0). Similarly, given a collection of 


\section{NON-ADDITIVE GEOMETRY}

symbols $\left\{f_{i}=f_{Y_{i}, X_{i}}=\left(\operatorname{id}_{Y_{i}}, \operatorname{id}_{X_{i}}\right) / \sim\right\}$, we can form the sum

$$
\coprod_{i \in I} A \cdot f_{i}
$$

of the free $A$-modules on the $f_{i}$, and

$$
\begin{aligned}
\operatorname{Hom}_{A-\mathcal{M o d}}\left(\coprod_{i \in I} A \cdot f_{i}, N\right) & =\left\{\left(n_{i}\right)_{i \in I} \mid n_{i} \in N_{Y_{i}, X_{i}}\right\} \\
& =\operatorname{Hom}_{\mathcal{S e t} /|\mathbb{F}| \times|\mathbb{F}|}\left(\left\{f_{i}\right\}_{i \in I}, \coprod_{Y, X \in|\mathbb{F}|} M_{Y, X}\right),
\end{aligned}
$$

i.e. the functor taking $\left\{f_{i}\right\}$ to $\amalg A \cdot f_{i}$ is the left-adjoint to the forgetful functor from $A$-modules to sets over $|\mathbb{F}| \times|\mathbb{F}|$, which takes an $A$-module $M$ to the $|\mathbb{F}| \times|\mathbb{F}|$ set

$$
\coprod_{Y, X \in|\mathbb{F}|} M_{Y, X}
$$

Tensor product. $\quad$ Let $M_{0}, M_{1}, N$ be $A$-modules. A collection of maps

$$
f=\left\{f_{Y_{0}, X_{0} ; Y_{1}, X_{1}}:\left(M_{0}\right)_{Y_{0}, X_{0}} \times\left(M_{1}\right)_{Y_{1}, X_{1}} \rightarrow N_{Y_{0} \otimes Y_{1}, X_{0} \otimes X_{1}}\right\}
$$

is called $A$-bilinear if it satisfies

$$
\begin{gathered}
f\left(m_{0} \oplus m_{0}^{\prime}, m_{1}\right)=f\left(m_{0}, m_{1}\right) \oplus f\left(m_{0}^{\prime}, m_{1}\right), \\
f\left(m_{0}, m_{1} \oplus m_{1}^{\prime}\right)=f\left(m_{0}, m_{1}\right) \oplus f\left(m_{0}, m_{1}^{\prime}\right), \\
f\left(a \otimes m_{0}, m_{1}\right)=a \otimes f\left(m_{0}, m_{1}\right)=f\left(m_{0}, a \otimes m_{1}\right), \\
f\left(a_{0} \circ m_{0} \circ a_{0}^{\prime}, a_{1} \circ m_{1} \circ a_{1}^{\prime}\right)=\left(a_{0} \otimes a_{1}\right) \circ f\left(m_{0}, m_{1}\right) \circ\left(a_{0}^{\prime} \otimes a_{1}^{\prime}\right) .
\end{gathered}
$$

We denote by $\operatorname{Bilin}\left(M_{0}, M_{1} ; N\right)$ the set of all such $f$. We can similarly define $\operatorname{Bilin}\left(M_{0}, \ldots, M_{l} ; N\right)$ as the set of all

$$
f=\left\{f_{Y_{0}, X_{0} ; \ldots ; Y_{l}, X_{l}}:\left(M_{0}\right)_{Y_{0}, X_{0}} \times \cdots \times\left(M_{l}\right)_{Y_{l}, X_{l}} \rightarrow N_{Y_{0} \otimes \cdots \otimes Y_{l}, X_{0} \otimes \cdots \otimes X_{l}}\right\}
$$

which are 'linear' in each variable. Note that if $f$ is bilinear with values in $N$, and $\varphi: N \rightarrow N^{\prime}$ is a homomorphism, then $\varphi \circ f$ is bilinear with values in $N^{\prime}$.

Lemma 3.20. There exists a universal bilinear map

$$
M_{0} \times M_{1} \rightarrow M_{0} \otimes_{A} M_{1},\left(m_{0}, m_{1}\right) \mapsto m_{0} \otimes m_{1},
$$

such that

$$
\operatorname{Bilin}\left(M_{0}, M_{1} ; N\right)=\operatorname{Hom}_{A-\mathcal{M o d}}\left(M_{0} \otimes M_{1}, N\right) .
$$

Proof. The usual construction of the tensor product goes through. We form the free $A$-module

$$
\coprod_{m_{i} \in M_{i}} A \cdot f\left(m_{0}, m_{1}\right)
$$

Here $m_{i}$ runs through the elements of $M_{i}, i=0,1$, and if $m_{i} \in\left(M_{i}\right)_{Y_{i}, X_{i}}$ we view $f\left(m_{0}, m_{1}\right)$ as a formal arrow from $X_{0} \otimes X_{1}$ to $Y_{0} \otimes Y_{1}$. We divide this free module by the equivalence $A$-module generated by the relations (3.19.1)-(3.19.4).

We write $m_{0} \otimes m_{1}$ for the image of $f\left(m_{0}, m_{1}\right)$ in $M_{0} \otimes_{A} M_{1}$.

We can similarly construct $M_{0} \otimes \cdots \otimes M_{l}$ so that

$$
\operatorname{Bilin}\left(M_{0}, \ldots, M_{l} ; N\right)=\operatorname{Hom}_{A-\mathcal{M o d}}\left(M_{0} \otimes \cdots \otimes M_{l}, N\right) .
$$




\section{J. Shai HARAN}

Proposition 3.21. There are canonical isomorphisms

$$
\begin{gathered}
M \otimes N \cong N \otimes M, \quad m \otimes n \mapsto n \otimes m, \\
(M \otimes N) \otimes L \cong M \otimes(N \otimes L) \cong M \otimes N \otimes L, \\
(m \otimes n) \otimes l \mapsto m \otimes(n \otimes l) \mapsto m \otimes n \otimes l, \\
M \otimes A=M .
\end{gathered}
$$

If $\varphi_{i}: M_{i} \rightarrow N_{i}$ are homomorphisms of $A$-modules, we get a homomorphism

$$
\begin{gathered}
\varphi_{0} \otimes \varphi_{1}: M_{0} \otimes M_{1} \rightarrow N_{0} \otimes N_{1}, \\
\varphi_{0} \otimes \varphi_{1}\left(m_{0} \otimes m_{1}\right) \mapsto \varphi_{0}\left(m_{0}\right) \otimes \varphi_{1}\left(m_{1}\right) .
\end{gathered}
$$

These are functorial,

$$
\operatorname{id}_{M_{0}} \otimes \operatorname{id}_{M_{1}}=\operatorname{id}_{M_{0} \otimes M_{1}}
$$

and for $\psi_{i}: N_{i} \rightarrow L_{i}$,

$$
\left(\psi_{0} \otimes \psi_{1}\right) \circ\left(\varphi_{0} \otimes \varphi_{1}\right)=\left(\psi_{0} \circ \varphi_{0}\right) \otimes\left(\psi_{1} \circ \varphi_{1}\right) .
$$

Proof. The usual proof using the universal property goes through.

Base change. Let $\varphi: A \rightarrow B$ be a homomorphism of $\mathbb{F}$-rings. If $N$ is a $B$-module, we can consider $N$ as an $A$-module via $\varphi$ :

$$
\begin{gathered}
a \circ n \circ a^{\prime}:=\varphi(a) \circ n \circ \varphi\left(a^{\prime}\right), \quad a, a^{\prime} \in A, n \in N, \\
a \otimes n:=\varphi(a) \otimes n, \quad a \in A, n \in N .
\end{gathered}
$$

We denote this $A$-module by $\varphi^{*} N$, or by $N_{\varphi}$.

Given an $A$-module $M$, form

$$
\left(M_{B}\right)_{Y, X}=\left\{\left(b, m, b^{\prime}\right) \mid b \in B_{Y, Y^{\prime}}, b^{\prime} \in B_{X^{\prime}, X}, m \in M_{Y^{\prime}, X^{\prime}}\right\} / \sim,
$$

where $\sim$ is the equivalence relation generated by

$$
\begin{gathered}
\left(b \circ \varphi(a), m, b^{\prime}\right) \sim\left(b, a \circ m, b^{\prime}\right), \quad\left(b, m, \varphi(a) \circ b^{\prime}\right) \sim\left(b, m \circ a, b^{\prime}\right), \\
\left(b \circ\left(c \otimes \operatorname{id}_{Y^{\prime}} \oplus \operatorname{id}_{Y^{\prime \prime}}\right),\left(\operatorname{id}_{Z} \otimes m^{\prime}\right) \oplus m^{\prime \prime}, b^{\prime}\right) \sim\left(b,\left(\operatorname{id}_{W} \otimes m^{\prime}\right) \oplus m^{\prime \prime},\left(c \otimes \operatorname{id}_{X^{\prime}} \oplus \operatorname{id}_{X^{\prime \prime}}\right) \circ b^{\prime}\right) \\
\text { for } c \in B_{W, Z}, m^{\prime} \in M_{Y^{\prime}, X^{\prime}}, m^{\prime \prime} \in M_{Y^{\prime \prime}, X^{\prime \prime}} .
\end{gathered}
$$

Let $\left(b, m, b^{\prime}\right) / \sim$ denote the equivalence class containing $\left(b, m, b^{\prime}\right)$, and define

$$
\begin{gathered}
\bar{b} \circ\left(b, m, b^{\prime}\right) / \sim \circ \bar{b}^{\prime}=\left(\bar{b} \circ b, m, b^{\prime} \circ \bar{b}^{\prime}\right) / \sim, \\
\left(b, m, b^{\prime}\right) / \sim \oplus\left(\bar{b}, \bar{m}, \bar{b}^{\prime}\right) / \sim=\left(b \oplus \bar{b}, m \oplus \bar{m}, b^{\prime} \oplus \bar{b}^{\prime}\right) / \sim, \\
\bar{b} \otimes\left(b, m, b^{\prime}\right) / \sim=\left(\bar{b} \otimes b, \operatorname{id}_{\bar{X}} \otimes m, \mathrm{id}_{\bar{X}} \otimes b^{\prime}\right) / \sim, \bar{b} \in B_{\bar{Y}, \bar{X}} .
\end{gathered}
$$

Proposition 3.23. These operations are well defined, independent of representatives. They make $M_{B}$ into a $B$-module; and $M \mapsto M_{B}$ is a functor from $A$-Mod to $B$-Mod, for $f: M \rightarrow M^{\prime}$ corresponds to $f_{B}: M_{B} \rightarrow M_{B}^{\prime}$,

$$
f_{B}\left(\left(b, m, b^{\prime}\right) / \sim\right)=\left(b, f(m), b^{\prime}\right) / \sim .
$$

This functor is the left-adjoint to the functor $\varphi^{*}$,

$$
\begin{gathered}
\operatorname{Hom}_{A-\mathcal{M o d}}\left(M, \varphi^{*} N\right)=\operatorname{Hom}_{B-\mathcal{M o d}}\left(M_{B}, N\right), \\
f \mapsto f\left(\left(b, m, b^{\prime}\right) / \sim\right)=b \circ f(m) \circ b^{\prime} .
\end{gathered}
$$




\section{NON-ADDITIVE GEOMETRY}

Proof. The proof is similar to those for Propositions 3.17 and 3.18. That (3.23.3) is well defined follows from associativity. The operation $\oplus$ of (3.23.4) is well defined with respect to (3.23.1):

$$
\begin{aligned}
& \left((b \circ \varphi(a)) \oplus \bar{b}, m \oplus \bar{m}, b^{\prime} \oplus \bar{b}^{\prime}\right) / \sim \\
& \quad=\left((b \oplus \bar{b}) \circ \varphi(a \oplus \mathrm{id}), m \oplus \bar{m}, b^{\prime} \oplus \bar{b}^{\prime}\right) / \sim \\
& \quad=\left(b \oplus \bar{b},(a \circ m) \oplus \bar{m}, b^{\prime} \oplus \bar{b}^{\prime}\right) / \sim .
\end{aligned}
$$

It is well defined with respect to $(3.23 .2)$ :

$$
\begin{aligned}
((b & \left.\left.\circ\left(c \otimes \mathrm{id}_{Y^{\prime}} \oplus \mathrm{id}_{Y^{\prime \prime}}\right)\right) \oplus \bar{b}, \mathrm{id}_{Z} \otimes m^{\prime} \oplus m^{\prime \prime} \oplus \bar{m}, b^{\prime} \oplus \bar{b}^{\prime}\right) / \sim \\
& =\left((b \oplus \bar{b}) \circ\left(c \otimes \mathrm{id}_{Y^{\prime}} \oplus \mathrm{id}_{Y^{\prime \prime}} \oplus \mathrm{id}_{\bar{Y}}\right), \mathrm{id}_{Z} \otimes m^{\prime} \oplus m^{\prime \prime} \oplus \bar{m}, b^{\prime} \oplus \bar{b}^{\prime}\right) / \sim \\
& =\left(b \oplus \bar{b}, \mathrm{id}_{Z} \otimes m^{\prime} \oplus m^{\prime \prime} \oplus \bar{m},\left(c \otimes \mathrm{id}_{X^{\prime}} \oplus \operatorname{id}_{X^{\prime \prime}} \oplus \mathrm{id}_{\bar{X}}\right) \circ\left(b^{\prime} \oplus \bar{b}^{\prime}\right)\right) / \sim \\
& =\left(b \oplus \bar{b}, \mathrm{id}_{Z} \otimes m^{\prime} \oplus m^{\prime \prime} \oplus \bar{m},\left(\left(c \otimes \mathrm{id}_{X^{\prime}} \oplus \mathrm{id}_{X^{\prime \prime}}\right) \circ b^{\prime}\right) \oplus \bar{b}^{\prime}\right) / \sim .
\end{aligned}
$$

The operation $\otimes$ of (3.23.5) is well defined with respect to (3.23.1):

$$
\begin{aligned}
(\bar{b} & \left.\otimes(b \circ \varphi(a)), \mathrm{id}_{\bar{X}} \otimes m, \mathrm{id}_{\bar{X}} \otimes b^{\prime}\right) / \sim \\
& =\left((\bar{b} \otimes b) \circ \varphi\left(\mathrm{id}_{\bar{X}} \otimes a\right), \mathrm{id}_{\bar{X}} \otimes m, \mathrm{id}_{\bar{X}} \otimes b^{\prime}\right) / \sim \\
& =\left(\bar{b} \otimes b, \mathrm{id}_{\bar{X}} \otimes(a \circ m), \mathrm{id}_{\bar{X}} \otimes b^{\prime}\right) / \sim .
\end{aligned}
$$

It is well defined with respect to $(3.23 .2)$ :

$$
\begin{aligned}
(\bar{b} & \left.\otimes\left(b \circ\left(c \otimes \operatorname{id}_{Y^{\prime}} \oplus \operatorname{id}_{Y^{\prime \prime}}\right)\right), \mathrm{id}_{\bar{X}} \otimes\left(\mathrm{id}_{Z} \otimes m^{\prime} \oplus m^{\prime \prime}\right), \mathrm{id}_{\bar{X}} \otimes b^{\prime}\right) / \sim \\
& =\left((\bar{b} \otimes b) \circ \mathrm{id}_{\bar{X}} \otimes\left(c \otimes \operatorname{id}_{Y^{\prime}} \oplus \operatorname{id}_{Y^{\prime \prime}}\right), \mathrm{id}_{\bar{X}} \otimes\left(\operatorname{id}_{Z} \otimes m^{\prime} \oplus m^{\prime \prime}\right), \mathrm{id}_{\bar{X}} \otimes b^{\prime}\right) / \sim \\
& =\left(\bar{b} \otimes b, \mathrm{id}_{\bar{X}} \otimes\left(\operatorname{id}_{W} \otimes m^{\prime} \oplus m^{\prime \prime}\right),\left(\mathrm{id}_{\bar{X}} \otimes\left(c \otimes \operatorname{id}_{X^{\prime}} \oplus \operatorname{id}_{X^{\prime \prime}}\right)\right) \circ\left(\operatorname{id}_{\bar{X}} \otimes b^{\prime}\right)\right) / \sim \\
& =\left(\bar{b} \otimes b, \mathrm{id}_{\bar{X}} \otimes\left(\operatorname{id}_{W} \otimes m^{\prime} \oplus m^{\prime \prime}\right), \mathrm{id}_{\bar{X}} \otimes\left(\left(c \otimes \operatorname{id}_{X^{\prime}} \oplus \operatorname{id}_{X^{\prime \prime}}\right) \circ b^{\prime}\right)\right) / \sim .
\end{aligned}
$$

One then checks the axioms for a $B$-module. In particular for the functoriality of $\otimes,(3.1 .11)$, we have 'on the left':

$$
\begin{aligned}
(\bar{c} & \otimes c) \circ\left(\bar{b} \otimes\left(b, m, b^{\prime}\right) / \sim\right) \\
& =\left((\bar{c} \otimes c) \circ(\bar{b} \otimes b), \mathrm{id}_{\bar{X}} \otimes m, \mathrm{id}_{\bar{X}} \otimes b^{\prime}\right) / \sim \\
& =\left((\bar{c} \circ \bar{b}) \otimes(c \circ b), \mathrm{id}_{\bar{X}} \otimes m, \mathrm{id}_{\bar{X}} \otimes b^{\prime}\right) / \sim \\
& =(\bar{c} \circ \bar{b}) \otimes\left(c \circ\left(b, m, b^{\prime}\right) / \sim\right) ;
\end{aligned}
$$

and 'on the right': for $\bar{b} \in B_{\bar{Y}, \bar{X}}, \bar{c} \in B_{\bar{X}, \bar{Z}}, m \in M_{Y^{\prime}, X^{\prime}}$

$$
\begin{aligned}
(\bar{b} & \left.\otimes\left(b, m, b^{\prime}\right) / \sim\right) \circ(\bar{c} \otimes c) \\
& =\left(\bar{b} \otimes b, \mathrm{id}_{\bar{X}} \otimes m,\left(\mathrm{id}_{\bar{X}} \otimes b^{\prime}\right) \circ(\bar{c} \otimes c)\right) / \sim \\
& =\left(\bar{b} \otimes b, \mathrm{id}_{\bar{X}} \otimes m,\left(\bar{c} \otimes \mathrm{id}_{X^{\prime}}\right) \circ\left(\mathrm{id}_{\bar{Z}} \otimes\left(b^{\prime} \circ c\right)\right)\right) / \sim \\
& =\left((\bar{b} \otimes b) \circ\left(\bar{c} \otimes \operatorname{id}_{Y^{\prime}}\right), \mathrm{id}_{\bar{Z}} \otimes m, \mathrm{id}_{\bar{Z}} \otimes\left(b^{\prime} \circ c\right)\right) / \sim \\
& =\left((\bar{b} \circ \bar{c}) \otimes b, \mathrm{id}_{\bar{Z}} \otimes m, \mathrm{id}_{\bar{Z}} \otimes\left(b^{\prime} \circ c\right)\right) / \sim \\
& =(\bar{b} \circ \bar{c}) \otimes\left(\left(b, m, b^{\prime}\right) / \sim \circ c\right) .
\end{aligned}
$$

That completes the proof.

The functor $M \mapsto M_{B}$ commutes with sums and direct limits,

$$
\left(\coprod_{i} M_{i}\right)_{B}=\coprod_{i}\left(M_{i}\right)_{B},\left(\stackrel{\lim }{\longrightarrow} M_{\lambda}\right)_{B}=\underline{\lim }\left(M_{\lambda}\right)_{B},
$$




\section{J. Shai HARAN}

with tensor products,

$$
\left(M \otimes_{A} N\right)_{B}=M_{B} \otimes_{B} N_{B}
$$

with the formation of free module,

$$
\left(\coprod_{i} A \cdot f_{i}\right)_{B}=\coprod_{i} B \cdot f_{i}
$$

and with cokernels: for $A$-module homomorphism $\varphi: M^{\prime} \rightarrow M$,

$$
\left(M / \varphi\left(M^{\prime}\right)\right)_{B}=M_{B} / \varphi_{B}\left(M_{B}^{\prime}\right) .
$$

Remark 3.23.11. We can consider $B$ as an $A$-module and form the tensor product $B \otimes_{A} M$, this map to $M_{B}$; but unlike the case of commutative rings, this map is not an isomorphism.

\section{Ideals and primes}

We define four notions of 'ideals' (ideal, $E$-ideal, $H$-ideal, $H$ - $E$-ideals), hence four notions of primes. We get four functors from $\mathbb{F}$-R ings to compact sober topological spaces, taking an $\mathbb{F}$-ring $A$ to the following commutative square of spaces.

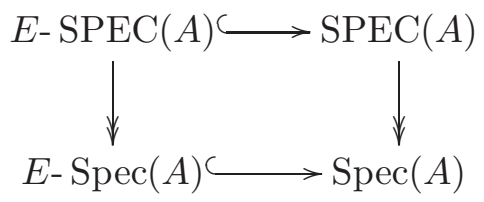

\section{1 $\boldsymbol{H}$-ideals and primes}

Let $A$ be an $\mathbb{F}$-ring.

Definition 4.1. An ideal $\mathfrak{a} \subseteq A$ is called homogeneous if it is generated by $\mathfrak{a}_{[1],[1]}$ (i.e., $\mathfrak{a}$ is the smallest ideal containing $\left.\mathfrak{a}_{[1],[1]}\right)$. A subset $\mathfrak{A} \subseteq A_{[1],[1]}$ is called an $H$-ideal if for

$$
a_{1}, \ldots, a_{n} \in \mathfrak{A}, \quad b \in A_{[1],[n]}, \quad b^{\prime} \in A_{[n],[1]}: b \circ\left(a_{1} \oplus \cdots \oplus a_{n}\right) \circ b^{\prime} \in \mathfrak{A} .
$$

If $\mathfrak{a}$ is a homogeneous ideal, $\mathfrak{a}_{[1],[1]}$ is an $H$-ideal. If $\mathfrak{A}$ is an $H$-ideal it generates a homogeneous ideal $\mathfrak{a}$, and $\mathfrak{a}_{[1],[1]}=\mathfrak{A}$. Hence there is one-to-one order-preserving correspondence between homogeneous ideals and $H$-ideals. We denote by $H$-id $(A)$ the set of $H$-ideals.

Proposition 4.1.1. Given $a_{1}, \ldots, a_{n} \in A_{[1],[1]}$ they generate the $H$-ideal

$$
\left(a_{1}, \ldots, a_{n}\right)=\left\{b \circ\left(\bigoplus_{i} a_{i} \cdot \mathrm{id}_{X_{i}}\right) \circ b^{\prime} \mid b \in A_{[1], \oplus X_{i}}, b^{\prime} \in A_{\oplus X_{i},[1]}\right\} .
$$

In particular for $a \in A_{[1],[1]},(a)=a \cdot A_{[1],[1]}$ are just the multiples of $a$. We have the zero ideal $(0)=\{0\}$, and the unit ideal $(1)=A_{[1],[1]}$.

Proof. Given $b \in A_{[1],[m]}, b^{\prime} \in A_{[m],[1]}, b_{j} \circ\left(\bigoplus_{i} a_{i} \cdot \operatorname{id}_{X_{i j}}\right) \circ b_{j}^{\prime} \in\left(a_{1}, \ldots, a_{n}\right), j=1, \ldots, m$, we have

$b \circ\left(\bigoplus_{j} b_{j} \circ\left(\bigoplus_{i} a_{i} \cdot \mathrm{id}_{X_{i j}}\right) \circ b_{j}^{\prime}\right) \circ b^{\prime}=\left(b \circ \bigoplus_{j} b_{j}\right) \circ\left(\bigoplus_{i} a_{i} \cdot \operatorname{id}_{\bigoplus_{j} X_{i j}}\right) \circ\left(\bigoplus_{j} b_{j}^{\prime} \circ b^{\prime}\right) \in\left(a_{1}, \ldots, a_{n}\right)$.

Given $\mathfrak{a}_{i} \in H$-id $(A), i \in I$, the intersection $\bigcap_{i} \mathfrak{a}_{i}$ is again an $H$-ideal. The sum $\sum \mathfrak{a}_{i}$ is the $H$-ideal generated by $\bigcup \mathfrak{a}_{i}$,

$$
\sum \mathfrak{a}_{i}=\left\{b \circ\left(\bigoplus_{j} a_{j}\right) \circ b^{\prime} \mid a_{j} \in \bigcup \mathfrak{a}_{i}\right\}
$$




\section{NON-ADDITIVE GEOMETRY}

The product $\mathfrak{a} \cdot \mathfrak{a}^{\prime}$ of two $H$-ideals is the $H$-ideal generated by the product of elements of these ideals,

$$
\mathfrak{a} \cdot \mathfrak{a}^{\prime}=\left\{b \circ\left(\bigoplus_{j} a_{j} \cdot a_{j}^{\prime}\right) \circ b^{\prime} \mid a_{j} \in \mathfrak{a}, a_{j}^{\prime} \in \mathfrak{a}^{\prime}\right\} .
$$

Proposition 4.1.4. For an $A$-module $M$, and for an element $m \in M_{Y, X}$, we have the $H$-ideal

$$
\operatorname{ann}_{A}(m)=\left\{a \in A_{[1],[1]} \mid a \cdot m=0\right\} .
$$

Proof. Indeed, if $a_{i} \cdot m=0$, then

$$
\left(b \circ\left(\bigoplus a_{i}\right) \circ b^{\prime}\right) \cdot m=\left(b \circ\left(\bigoplus_{i} a_{i}\right) \circ b^{\prime}\right) \otimes\left(\operatorname{id}_{Y} \circ m \circ \operatorname{id}_{X}\right)=\left(b \otimes \operatorname{id}_{Y}\right) \circ\left(\bigoplus a_{i} \cdot m\right) \circ\left(b^{\prime} \otimes \operatorname{id}_{X}\right)=0 .
$$

Similarly, for $m, m^{\prime} \in M_{Y, X}$ we have the $H$-ideal

$$
\operatorname{ann}_{A}\left(m, m^{\prime}\right)=\left\{a \in A_{[1],[1]} \mid a \cdot m=a \cdot m^{\prime}\right\} .
$$

If $M_{0}, M_{1} \subseteq M$ are $A$-submodules, we have the $H$-ideal

$$
\left(M_{0}: M_{1}\right)=\left\{a \in A_{[1],[1]} \mid a \cdot M_{1} \subseteq M_{0}\right\} .
$$

Let $\varphi: A \rightarrow B$ be a homomorphism of $\mathbb{F}$-rings. If $\mathfrak{b} \in H-\operatorname{id}(B)$ then $\varphi^{*}(\mathfrak{b})=\varphi^{-1}(\mathfrak{b}) \in H$-id $(A)$, and we have the map

$$
\varphi^{*}: H-\mathrm{id}(B) \rightarrow H-\mathrm{id}(A), \quad \mathfrak{b} \mapsto \varphi^{-1}(\mathfrak{b}) .
$$

If $\mathfrak{a} \in H-\operatorname{id}(A), \varphi(\mathfrak{a})$ generates the $H$-ideal $\varphi_{*}(\mathfrak{a})$,

$$
\varphi_{*}: H-\operatorname{id}(A) \rightarrow H-\operatorname{id}(B), \quad \mathfrak{a} \mapsto \varphi_{*}(\mathfrak{a})=\left\{b \circ\left(\bigoplus \varphi\left(a_{i}\right)\right) \circ b^{\prime}\right\} .
$$

Proposition 4.2. We have the following:

(1) $\mathfrak{a} \subseteq \varphi^{*} \varphi_{*} \mathfrak{a}, \mathfrak{a} \in H-\operatorname{id}(A)$;

(2) $\mathfrak{b} \supseteq \varphi_{*} \varphi^{*} \mathfrak{b}, \mathfrak{b} \in H-\operatorname{id}(B)$;

(3) $\varphi^{*} \mathfrak{b}=\varphi^{*} \varphi_{*} \varphi^{*} \mathfrak{b}, \varphi_{*} \mathfrak{a}=\varphi_{*} \varphi^{*} \varphi_{*} \mathfrak{a}$;

(4) there is a bijection, via $\mathfrak{a} \mapsto \varphi_{*} \mathfrak{a}$ (with inverse map $\mathfrak{b} \mapsto \varphi^{*} \mathfrak{b}$ ), from the set

$$
\left\{\mathfrak{a} \in H-\operatorname{id}(A) \mid \varphi^{*} \varphi_{*} \mathfrak{a}=\mathfrak{a}\right\}=\left\{\varphi^{*} \mathfrak{b} \mid \mathfrak{b} \in H-\operatorname{id}(B)\right\}
$$

to the set

$$
\left\{\mathfrak{b} \in H-\operatorname{id}(B) \mid \varphi_{*} \varphi^{*} \mathfrak{b}=\mathfrak{b}\right\}=\left\{\varphi_{*} \mathfrak{a} \mid \mathfrak{a} \in H-\operatorname{id}(A)\right\} .
$$

Proof. The proofs of these are straightforward.

Definition 4.3.1. For $\mathfrak{A} \in H$-id $(A)$, we have the homogeneous ideal $\mathfrak{a}$ generated by $\mathfrak{A}$, and the equivalence ideal $E \mathfrak{a}=E \mathfrak{A}$ it generates (cf., Definition 2.19). We write $A / \mathfrak{A}$ for $A / E \mathfrak{A}$, and let $\pi: A \rightarrow A / \mathfrak{A}$ be the canonical homomorphism.

Proposition 4.3.2. We have a one-to-one order-preserving correspondence

$$
\pi^{*}: H-\operatorname{id}(A / \mathfrak{A}) \stackrel{\sim}{\longrightarrow}\{\mathfrak{b} \in H-\operatorname{id}(A) \mid \mathfrak{b} \text { is } E \mathfrak{A} \text {-stable }\} ;
$$

here $\mathfrak{b}$ is EA-stable if, for $a \in \mathfrak{A}, Z \in|\mathbb{F}|$,

$$
b \circ\left(\operatorname{id}_{Z} \oplus a\right) \circ b^{\prime} \in \mathfrak{b} \Leftrightarrow b \circ\left(\operatorname{id}_{Z} \oplus 0\right) \circ b^{\prime} \in \mathfrak{b} .
$$

Proof. The proof is clear. 


\section{J. Shai HaRAN}

We often say $H$-ideal $\mathfrak{a}$ meaning proper $H$-ideal, i.e. $\mathfrak{a} \neq(1)$. Since the union of a chain of $H$-ideals is again an $H$-ideal, an application of Zorn's lemma gives the following result.

Theorem 4.4. Every $\mathbb{F}$-ring contains a maximal (proper) $H$-ideal. We denote by $\operatorname{Max}(A)$ the set of maximal $H$-ideals.

Definition 4.5. An $H$-ideal $\mathfrak{p} \subseteq A_{[1],[1]}$ is called prime $H$-ideal (or in short 'prime') if $A_{[1],[1]} \backslash \mathfrak{p}$ is multiplicative closed:

$$
f, g \in A_{[1],[1]} \backslash \mathfrak{p} \Rightarrow f \cdot g \notin \mathfrak{p} .
$$

We denote by $\operatorname{Spec} A$ the set of prime $H$-ideals. For a homomorphism of $\mathbb{F}$-rings $\varphi: A \rightarrow B$, the pull-back $\varphi^{*}=\varphi^{-1}$ induces a map

$$
\varphi^{*}=\operatorname{Spec}(\varphi): \operatorname{Spec} B \rightarrow \operatorname{Spec} A .
$$

Proposition 4.6. If $\mathfrak{m}$ is a maximal $H$-ideal then $\mathfrak{m}$ is prime.

Proof. If $f, g \in A_{[1],[1]} \backslash \mathfrak{m}$, the $H$-ideals (cf. (4.1.2)) $(f)+\mathfrak{m},(g)+\mathfrak{m}$ are the unit $H$-ideals. So we can write (cf. (4.1.1)),

$$
1=b_{1} \circ\left(\operatorname{id}_{X_{1}} \cdot f \oplus m_{1}\right) \circ b_{1}^{\prime}, \quad 1=b_{2} \circ\left(\operatorname{id}_{X_{2}} \cdot g \oplus m_{2}\right) \circ b_{2}^{\prime},
$$

with $m_{i} \in(\mathfrak{m})=\{$ ideal generated by $\mathfrak{m}\}, m_{i} \in(\mathfrak{m})_{Z_{i}, W_{i}}, b_{i} \in A_{[1], X_{i} \oplus Z_{i}}, b_{i}^{\prime} \in A_{X_{i} \oplus W_{i},[1]}$. So we have

$$
1=1 \cdot 1=\left(b_{1} \otimes b_{2}\right) \circ\left(\mathrm{id}_{X_{1} \otimes X_{2}} \cdot f \cdot g \oplus m\right) \circ\left(b_{1}^{\prime} \otimes b_{2}^{\prime}\right),
$$

with

$$
m=\left(\mathrm{id}_{X_{1}} \cdot f \otimes m_{2}\right) \oplus\left(m_{1} \otimes \mathrm{id}_{X_{2}} \cdot g\right) \oplus\left(m_{1} \otimes m_{2}\right) \in(\mathfrak{m}),
$$

hence $f \cdot g \notin \mathfrak{m}$.

More generally, given an $H$-ideal $\mathfrak{a} \in H$-id $(A)$, and given $f \in A_{[1],[1]}$ such that $f^{n} \notin \mathfrak{a}, n \geqslant 0$, let $\mathfrak{m}$ be a maximal element of the set

$$
\left\{\mathfrak{b} \in H-\operatorname{id}(A) \mid \mathfrak{b} \supseteq \mathfrak{a}, f^{n} \notin \mathfrak{b} \forall n \geqslant 0\right\} .
$$

Claim 4.6.2. We claim that $\mathfrak{m}$ is prime.

Proof. If $g_{i} \in A_{[1],[1]} \backslash \mathfrak{m}, i=1,2$, we have for some $n_{i} \geqslant 0, f^{n_{i}}=b_{i} \circ\left(\mathrm{id}_{X_{i}} \cdot g_{i} \oplus m_{i}\right) \circ b_{i}^{\prime}$, hence

$$
f^{n_{1}+n_{2}}=\left(b_{1} \otimes b_{2}\right) \circ\left(\mathrm{id}_{X_{1} \otimes X_{2}} \cdot g_{1} \cdot g_{2} \oplus m\right) \circ\left(b_{1}^{\prime} \otimes b_{2}^{\prime}\right),
$$

with $m \in\{$ ideal generated by $\mathfrak{m}\}$, hence $g_{1} \cdot g_{2} \notin \mathfrak{m}$.

Definition 4.7. For $\mathfrak{a} \in H$-id $(A)$, the radical is

$$
\sqrt{\mathfrak{a}}=\left\{f \in A_{[1],[1]} \mid f^{n} \in \mathfrak{a} \text { for some } n \geqslant 1\right\} .
$$

It is easy to see that $\sqrt{\mathfrak{a}}$ is an $H$-ideal. This also follows from the following proposition.

Proposition 4.7.1. We have

$$
\sqrt{\mathfrak{a}}=\bigcap_{\mathfrak{a} \subseteq \mathfrak{p}} \mathfrak{p}
$$

the intersection of prime $H$-ideals containing $\mathfrak{a}$.

Proof. If $f \in \sqrt{\mathfrak{a}}$, say $f^{n} \in \mathfrak{a}$, then for all primes $\mathfrak{p} \supseteq \mathfrak{a}, f \in \mathfrak{p}$. If $f \notin \sqrt{\mathfrak{a}}$, let $\mathfrak{m}$ be a maximal element of the set (4.6.1), it exists by Zorn's lemma, and it is prime by Claim 4.6.2, $\mathfrak{m} \supseteq \mathfrak{a}$ and $f \notin \mathfrak{m}$. 


\section{NON-ADDITIVE GEOMETRY}

Definition 4.8. For a set $\mathfrak{A} \subseteq A_{[1],[1]}$, we let

$$
V_{A}(\mathfrak{A})=\{\mathfrak{p} \in \operatorname{Spec} A \mid \mathfrak{p} \supseteq \mathfrak{A}\} .
$$

If $\mathfrak{a}$ is the $H$-ideal generated by $\mathfrak{A}, V_{A}(\mathfrak{A})=V_{A}(\mathfrak{a})$; we have

$$
\begin{gathered}
V_{A}(1)=\varnothing \text { (empty set), } V_{A}(0)=\operatorname{Spec} A, \\
V_{A}\left(\sum \mathfrak{a}_{i}\right)=\bigcap_{i} V_{A}\left(\mathfrak{a}_{i}\right), \mathfrak{a}_{i} \in H-\operatorname{id}(A), \\
V_{A}\left(\mathfrak{a} \cdot \mathfrak{a}^{\prime}\right)=V_{A}(\mathfrak{a}) \cup V_{A}\left(\mathfrak{a}^{\prime}\right) .
\end{gathered}
$$

Hence the sets $\left\{V_{A}(\mathfrak{a}) \mid \mathfrak{a} \in H\right.$-id $\left.(A)\right\}$ are the closed sets for a topology on $\operatorname{Spec} A$, the Zariski topology.

Definition 4.9. For $f \in A_{[1],[1]}$ we let

$$
D_{A}(f)=\operatorname{Spec}(A) \backslash V_{A}(f)=\{\mathfrak{p} \in \operatorname{Spec} A \mid f \notin \mathfrak{p}\} .
$$

We have

$$
\begin{aligned}
D_{A}\left(f_{1}\right) \cup D_{A}\left(f_{2}\right) & =D_{A}\left(f_{1} \cdot f_{2}\right), \\
\operatorname{Spec} A \backslash V_{A}(\mathfrak{a}) & =\bigcup_{f \in \mathfrak{a}} D_{A}(f) .
\end{aligned}
$$

Hence the sets $\left\{D_{A}(f) \mid f \in A_{[1],[1]}\right\}$ form a basis for the open sets in the Zariski topology. We have

$$
D_{A}(f)=\varnothing \quad \Leftrightarrow \quad f \in \bigcap_{\mathfrak{p} \in \operatorname{Spec} A} \mathfrak{p}=\sqrt{0} \quad \Leftrightarrow \quad f^{n}=0 \text { for some } n
$$

and we say $f$ is nilpotent. We have

$$
D_{A}(f)=\operatorname{Spec} A \quad \Leftrightarrow \quad(f)=(1) \quad \Leftrightarrow \quad \exists f^{-1} \in A_{[1],[1]}: f \cdot f^{-1}=1
$$

and we say $f$ is invertible. We denote by $G L_{[1]}(A)$ the (commutative) group of invertible elements. Definition. For a subset $X \subseteq \operatorname{Spec} A$, we have the associated $H$-ideal

$$
I(X)=\bigcap_{\mathfrak{p} \in X} \mathfrak{p}
$$

Proposition 4.10. We have

$$
\begin{gathered}
I V_{A}(\mathfrak{a})=\sqrt{\mathfrak{a}}, \\
V_{A} I(X)=\bar{X} \text {, the closure of } X \text { in the Zariski topology. }
\end{gathered}
$$

Proof. Equation (4.10.1) is just a restatement of Proposition 4.7.1. For (4.10.2), $V_{A} I(X)$ is clearly a closed set containing $X$, and if $C=V_{A}(\mathfrak{a})$ is a closed set containing $X$, then $\sqrt{\mathfrak{a}}=I V_{A}(\mathfrak{a}) \subseteq I(X)$, hence $C=V_{A}(\sqrt{\mathfrak{a}}) \supseteq V_{A} I(X)$.

Corollary 4.11. We have a one-to-one order-reversing correspondence between closed sets $X \subseteq$ $\operatorname{Spec} A$, and radical $H$-ideals $\mathfrak{a}$, via $X \mapsto I(X), V_{A}(\mathfrak{a}) \longleftrightarrow \mathfrak{a}$,

$$
\{X \subseteq \operatorname{Spec} A \mid \bar{X}=X\} \stackrel{1: 1}{\leftrightarrow}\{\mathfrak{a} \in H-\operatorname{id}(A) \mid \sqrt{\mathfrak{a}}=\mathfrak{a}\} .
$$

Under this correspondence the closed irreducible subsets correspond to the prime ideals. For $\mathfrak{p}_{0}, \mathfrak{p}_{1} \in$ $\operatorname{Spec} A, \mathfrak{p}_{0} \in \overline{\left\{\mathfrak{p}_{1}\right\}} \Leftrightarrow \mathfrak{p}_{0} \supseteq \mathfrak{p}_{1}$, we say that $\mathfrak{p}_{0}$ is a Zariski specialization of $\mathfrak{p}_{1}$, or that $\mathfrak{p}_{1}$ is a Zariski generalization of $\mathfrak{p}_{0}$. The space $\operatorname{Spec} A$ is sober : every closed irreducible subset $C$ has the form $C=V_{A}(\mathfrak{p})=\overline{\{\mathfrak{p}\}}$, and we call the (unique) prime $\mathfrak{p}$ the generic point of $C$. 


\section{J. Shai HARAN}

Proposition 4.12. The sets $D_{A}(f)$, and in particular $D_{A}(1)=\operatorname{Spec} A$, are compact (or 'quasicompact': we do not include Hausdorff in compactness).

Proof. Note that $D_{A}(f)$ is contained in the union $\bigcup_{i} D_{A}\left(g_{i}\right)$ if and only if $V_{A}(f) \supseteq \bigcap_{i} V_{A}\left(g_{i}\right)=$ $V_{A}(\mathfrak{a})$, where $\mathfrak{a}$ is the $H$-ideal generated by $\left\{g_{i}\right\}$, if and only if $\sqrt{f}=I V_{A}(f) \subseteq I V_{A}(\mathfrak{a})=\sqrt{\mathfrak{a}}$, if and only if $f^{n} \in \mathfrak{a}$ for some $n$, if and only if $f^{n}=b \circ\left(\bigoplus_{i} g_{i} \cdot \mathrm{id}_{X_{i}}\right) \circ b^{\prime}$, and in any such expression only a finite number of the $g_{i}$ are involved.

Let $\varphi: A \rightarrow B$ be a homomorphism of $\mathbb{F}$-rings, $\varphi^{*}:$ Spec $B \rightarrow \operatorname{Spec} A$ the associated pull-back map.

Proposition 4.13. We have

$$
\begin{gathered}
\varphi^{*-1}\left(D_{A}(f)\right)=D_{B}(\varphi(f)), \quad f \in A_{[1],[1]}, \\
\varphi^{*-1}\left(V_{A}(\mathfrak{a})\right)=V_{B}\left(\varphi_{*}(\mathfrak{a})\right), \quad \mathfrak{a} \in H-\operatorname{id}(A), \\
V_{A}\left(\varphi^{-1} \mathfrak{b}\right)=\overline{\varphi^{*}\left(V_{B}(\mathfrak{b})\right)}, \quad \mathfrak{b} \in H-\operatorname{id}(B) .
\end{gathered}
$$

Proof. The proofs of (4.13.1) and (4.13.2) are straightforward:

$\mathfrak{q} \in \varphi^{*-1}\left(D_{A}(f)\right) \quad \Leftrightarrow \quad \varphi^{*}(\mathfrak{q}) \in D_{A}(f) \quad \Leftrightarrow \quad f \notin \varphi^{-1}(\mathfrak{q}) \quad \Leftrightarrow \quad \varphi(f) \notin \mathfrak{q} \quad \Leftrightarrow \quad \mathfrak{q} \in D_{B}(\varphi(f))$,

$\mathfrak{q} \in \varphi^{*-1}\left(V_{A}(\mathfrak{a})\right) \quad \Leftrightarrow \quad \varphi^{*}(\mathfrak{q}) \in V_{A}(\mathfrak{a}) \quad \Leftrightarrow \quad \mathfrak{a} \subseteq \varphi^{-1}(\mathfrak{q}) \quad \Leftrightarrow \quad \varphi_{*}(\mathfrak{a}) \subseteq \mathfrak{q} \quad \Leftrightarrow \quad \mathfrak{q} \in V_{B}\left(\varphi_{*}(\mathfrak{a})\right)$.

For (4.13.3) we may assume $\mathfrak{b}=\sqrt{\mathfrak{b}}$ is radical since $V_{B}(\mathfrak{b})=V_{B}(\sqrt{\mathfrak{b}}), \varphi^{-1}(\sqrt{\mathfrak{b}})=\sqrt{\varphi^{-1}(\mathfrak{b})}$. Let $\mathfrak{a}=I\left(\varphi^{*}\left(V_{B}(\mathfrak{b})\right)\right)$, so that $V_{A}(\mathfrak{a})=\overline{\varphi^{*}\left(V_{B}(\mathfrak{b})\right)}$ by $(4.10 .2)$. We have

$$
\begin{aligned}
f \in \mathfrak{a} \quad & \Leftrightarrow f \in \mathfrak{p}, \quad \forall \mathfrak{p} \in \varphi^{*}\left(V_{B}(\mathfrak{b})\right) \quad \Leftrightarrow \quad f \in \varphi^{-1}(\mathfrak{q}), \quad \forall \mathfrak{q} \supseteq \mathfrak{b} \\
& \Leftrightarrow \varphi(f) \in \bigcap_{\mathfrak{q} \supseteq \mathfrak{b}} \mathfrak{q}=\sqrt{\mathfrak{b}}=\mathfrak{b} \quad \Leftrightarrow \quad f \in \varphi^{-1}(\mathfrak{b}) .
\end{aligned}
$$

It follows from (4.13.1), or from (4.13.2), that $\varphi^{*}=\operatorname{Spec}(\varphi)$ is continuous, hence $A \mapsto \operatorname{Spec} A$ is a contravariant functor from $\mathbb{F}$-rings to compact, sober, topological spaces.

Example 4.14.1. Let $A$ be a commutative ring, $\mathbb{F}(A)$ the associated $\mathbb{F}$-ring. An ideal $\mathfrak{a} \subseteq A=$ $\mathbb{F}(A)_{[1],[1]}$ is an $H$-ideal generating the homogeneous ideal $\mathbb{F}(\mathfrak{a}) \subseteq \mathbb{F}(A)$, and conversely an $H$-ideal is just an ideal of $A$. Under this correspondence the primes of $A$ correspond to the primes of $\mathbb{F}(A)$, and we have a homeomorphism with respect to the Zariski topologies:

$$
\operatorname{Spec} A=\operatorname{Spec} \mathbb{F}(A) .
$$

Example 4.14.2. Let $\eta: \mathbb{k} \rightarrow \mathbb{C}$ be a real or complex prime of a number field, and let $\mathcal{O}_{\mathbb{k}, \eta}$ denote the $\mathbb{F}$-ring of real or complex 'integers' of (2.16). Then

$$
\mathfrak{m}_{\eta}=\left\{\left.x \in \mathbb{k}|| x\right|_{\eta}<1\right\}
$$

is the (unique) maximal $H$-ideal of $\mathcal{O}_{\mathbb{k}, \eta}$, the closed point of $\operatorname{Spec} \mathcal{O}_{\mathbb{k}, \eta}$.

\section{$4.2 S$-prime ideals and $H$ - $E$-ideals}

Definition 4.15. A (non-homogeneous) ideal $\mathfrak{p} \subseteq A$ is called a strong prime ideal, or $S$-prime, if $A \backslash \mathfrak{p}$ is closed with respect to $\otimes$ :

$$
f \in(A \backslash \mathfrak{p})_{Y_{1}, X_{1}}, g \in(A \backslash \mathfrak{p})_{Y_{2}, X_{2}} \Rightarrow f \otimes g \in(A \backslash \mathfrak{p})_{Y_{1} \otimes Y_{2}, X_{1} \otimes X_{2}} .
$$

We let $\operatorname{SPEC}(A)$ denote the set of S-primes. 


\section{NON-ADDITIVE GEOMETRY}

Every $\mathbb{F}$-ring contains a maximal ideal, and every maximal ideal is S-prime; cf. Proposition 4.6. For an ideal $\mathfrak{a}$ we have

$$
\sqrt{\mathfrak{a}}=\left\{f \in A \mid f^{\otimes n} \in \mathfrak{a} \text { for some } n \geqslant 1\right\}=\bigcap_{\mathfrak{a} \subseteq \mathfrak{p}} \mathfrak{p},
$$

the intersection of S-primes containing $\mathfrak{a}$; cf. Proposition 4.7.1. On $\operatorname{SPEC}(A)$ we have the Zariski topology, with closed sets

$$
V_{A}(\mathfrak{a})=\{\mathfrak{p} \in \operatorname{SPEC}(A) \mid \mathfrak{p} \supseteq \mathfrak{a}\},
$$

where we can take $\mathfrak{a}$ to be an ideal of $A$; cf. Definition 4.8. A basis for the open sets is given by the sets

$$
D_{A}(f)=\operatorname{SPEC}(A) \backslash V_{A}(f)=\{\mathfrak{p} \in \operatorname{SPEC}(A) \mid f \notin \mathfrak{p}\},
$$

$f \in A$; cf. Definition 4.9. We have

$$
D_{A}(f)=\varnothing \quad \Leftrightarrow \quad f \in \bigcap_{\mathfrak{p} \in \operatorname{SPEC}(A)} \mathfrak{p}=\sqrt{0} \quad \Leftrightarrow \quad f^{\otimes n}=0 \text { for some } n,
$$

and we say $f$ is nilpotent. We have

$$
D_{A}(f)=\operatorname{SPEC} A \quad \Leftrightarrow \quad(f)=(1) \quad \Leftrightarrow \quad 1=a_{1} \circ\left(\operatorname{id}_{X} \otimes f\right) \circ a_{2}
$$

for some $a_{i} \in A$, and we say $f$ is $a$ unit. For a subset $X \subseteq \operatorname{SPEC}(A)$, we have the associated ideal

$$
I(X)=\bigcap_{\mathfrak{p} \in X} \mathfrak{p},
$$

and Proposition 4.10 holds. Similarly Corollary 4.11 and Propositions 4.12 and 4.13 remain true. We have a continuous map

$$
\operatorname{SPEC}(A) \rightarrow \operatorname{Spec}(A), \mathfrak{p} \mapsto \mathfrak{p}_{[1],[1]} .
$$

It is always surjective. For an $\mathbb{F}$-ring $\mathbb{F}(A)$ associated to a commutative ring $A$, ideals of $\mathbb{F}(A)$ correspond one-to-one with ideals of $A, S$-primes correspond to primes of $A$, and the map (4.15.6) is a homeomorphism.

Definition 4.16. A non-empty subset $\mathfrak{A} \subseteq A_{[1],[1]}$ will be called an $H$-E-ideal if, for $a_{i} \in \mathfrak{A}$,

$$
b \circ\left(\operatorname{id}_{Z} \oplus \bigoplus_{i} a_{i}\right) \circ b^{\prime} \in \mathfrak{A} \quad \Leftrightarrow \quad b \circ\left(\operatorname{id}_{Z} \oplus 0\right) \circ b^{\prime} \in \mathfrak{A} .
$$

We have $0 \in \mathfrak{A}$, and hence for $a_{i} \in \mathfrak{A}, i=1, \ldots, n, b \circ\left(\bigoplus a_{i}\right) \circ b^{\prime} \in \mathfrak{A}$, i.e. it is an $H$-ideal (take $Z=[0]$ in (4.16.1)). Thus an $H$-E-ideal $\mathfrak{A} \subseteq A_{[1],[1]}$ is just an $H$-ideal which is $E \mathfrak{A}$-stable. We denote by $H-E$-id $(A)$ the collection of $H$-E-ideals of $A$.

Given $\mathfrak{A} \in H$-id $(A)$, it generates the homogeneous ideal $\mathfrak{a}=\left\{b \circ\left(\bigoplus a_{i}\right) \circ b^{\prime} \mid a_{i} \in \mathfrak{A}\right\}$, which in turn generates the $E$-ideal $Z E \mathfrak{a} \in E$-sub $\operatorname{su}_{A}(A)$, which can be described as (cf., Lemma 3.13):

$$
\begin{aligned}
Z E \mathfrak{a}= & \left\{a \in A \mid \exists \text { path } a=c_{1}, \ldots, c_{l}=0, \text { with }\left\{c_{j}, c_{j+1}\right\}\right. \text { of the form } \\
& \left.\left\{b \circ\left(\operatorname{id}_{Z} \oplus \bigoplus_{i} a_{i}\right) \circ b^{\prime}, b \circ\left(\operatorname{id}_{Z} \oplus 0\right) \circ b^{\prime}\right\}, \text { with } a_{i} \in \mathfrak{A}\right\} .
\end{aligned}
$$

The $E$-ideal $Z E \mathfrak{a}=Z E \mathfrak{A}$ is generated as $E$-ideal by $(Z E \mathfrak{A})_{[1],[1]} \subseteq A_{[1],[1]}$ (by the explicit description $(4.16 .2)$ and the fact that $\left.(Z E \mathfrak{A})_{[1],[1]} \supseteq \mathfrak{A}\right)$; we call the $H$-E-ideal $(Z E \mathfrak{A})_{[1],[1]}$ the $E$-closure of $\mathfrak{A}$. We have $\mathfrak{A}=(Z E \mathfrak{A})_{[1],[1]}$ if and only if $\mathfrak{A}$ is an $H$-E-ideal. 


\section{J. Shai HARAN}

COROLlary 4.16.3. There is a one-to-one order-preserving correspondence between the set of equivalence ideal EA, generated by a subset $\mathfrak{A} \subseteq A_{[1],[1]}$

$$
\left\{\mathcal{E} \in \operatorname{equiv}_{A}(A) \mid \mathcal{E}=E\left((Z \mathcal{E})_{[1],[1]}\right)\right\}=\{E \mathfrak{A} \mid \mathfrak{A} \in H-\operatorname{id}(A)\},
$$

and the set of $H-E-\operatorname{id}(A)$

$$
H-E-\operatorname{id}(A)=\left\{\mathfrak{A} \in H-E-\operatorname{id}(A) \mid \mathfrak{A}=(Z E \mathfrak{A})_{[1],[1]}\right\} .
$$

It is given by:

$$
\mathcal{E} \mapsto(Z \mathcal{E})_{[1],[1]}, \quad E \mathfrak{A} \longleftrightarrow \mathfrak{A} .
$$

For $\mathfrak{A} \in H-E-\operatorname{id}(A)$, let $\pi: A \rightarrow A / \mathfrak{A}=A / E \mathfrak{A}$ denote the canonical projection, then $\mathfrak{A}=$ $\pi^{-1}\left(0_{[1],[1]}\right)$. We have the following proposition.

Proposition 4.17. There is a one-to-one order-preserving correspondence

$$
\begin{gathered}
\{\mathfrak{B} \in H-E-\operatorname{id}(A) \mid \mathfrak{B} \supseteq \mathfrak{A}\} \stackrel{1: 1}{\leftrightarrow} H-E-\operatorname{id}(A / \mathfrak{A}), \\
\pi^{-1}(\overline{\mathfrak{B}}) \longleftarrow \overline{\mathfrak{B}} .
\end{gathered}
$$

We can repeat most of our discussion of $H$-ideals using $H$-E-ideals. By Zorn's lemma, we get the next result.

Proposition 4.18. There exist maximal (proper) H-E-ideals.

Definition 4.19. We define $E$ - $\operatorname{Spec}(A)=\operatorname{Spec}(A) \cap H$-E-id $(A)$; its elements will be called E-primes. We have the following proposition.

Proposition 4.19.1. If $\mathfrak{m}$ is a maximal $H$-E-ideal then $\mathfrak{m}$ is E-prime.

More generally, given $\mathfrak{a} \in H$-E-id $(A), f \in A_{[1],[1]}$, such that $f^{n} \notin \mathfrak{a}$ for all $n$. By Zorn's lemma there exists a maximal element $\mathfrak{m}$ in the set

$$
\left\{\mathfrak{b} \in H-E-\operatorname{id}(A) \mid \mathfrak{b} \supseteq \mathfrak{a}, f^{n} \notin \mathfrak{b} \forall n\right\} .
$$

Claim 4.19.2. We claim that $\mathfrak{m}$ is E-prime.

Proof. For $x \in A_{[1],[1]} \backslash \mathfrak{m}$, the $H$-E-ideal generated by $\mathfrak{m}$ and $x$ contains some power $f^{n}$. Hence there is a path $f^{n}=c_{1}, \ldots, c_{l}=0$, with $\left\{c_{j}, c_{j+1}\right\}$ of the form $\left\{b \circ\left(\operatorname{id}_{Z} \oplus\left(x \cdot \operatorname{id}_{W}\right) \oplus m\right) \circ b^{\prime}, b \circ\left(\operatorname{id}_{Z} \oplus 0\right) \circ b^{\prime}\right\}$, with $m$ in the ideal generated by $\mathfrak{m}$. Let $y \in A_{[1],[1]}$ with $y \cdot x \in \mathfrak{m}$. Multiplying the path $\left\{c_{j}\right\}$ throughout by $y$ we see that $y \cdot f^{n} \in \mathfrak{m}$. If $y \in A_{[1],[1]} \backslash \mathfrak{m}$, we get similarly a path $f^{m}=d_{1}, \ldots, d_{l}=0$, with $\left\{d_{j}, d_{j+1}\right\}$ of the form $\left\{b \circ\left(\operatorname{id}_{Z} \oplus y \cdot \operatorname{id}_{W} \oplus m\right) \circ b^{\prime}, b \circ\left(\operatorname{id}_{Z} \oplus 0\right) \circ b^{\prime}\right\}$, and multiplying this path by $f^{n}$ we see that $f^{n} \cdot f^{m} \in \mathfrak{m}$, a contradiction.

Corollary 4.19.3. For $\mathfrak{A} \in H-E-\operatorname{id}(A)$,

$$
\sqrt{\mathfrak{A}}=\bigcap_{\mathfrak{A} \subseteq \mathfrak{p}} \mathfrak{p},
$$

the intersection of all E-primes containing $\mathfrak{A}$.

Example 4.20. For a commutative ring $A$, every $H$-ideal of $\mathbb{F}(A)$ is an $H$ - $E$-ideal,

$$
H-E-\operatorname{id}(\mathbb{F}(A))=H-\operatorname{id}(\mathbb{F}(A))=\text { ideals of } A \text {. }
$$

Hence every prime is an E-prime,

$$
E-\operatorname{Spec}(\mathbb{F}(A))=\operatorname{Spec}(\mathbb{F}(A))=\operatorname{Spec}(A) .
$$




\section{NON-ADDITIVE GEOMETRY}

Example 4.21. The maximal $H$-ideal $\mathfrak{m}_{\eta}$ of the $\mathbb{F}$-ring of real or complex integers $\mathcal{O}_{\mathbb{k}, \eta}$, cf. Example 4.14.2, is an $E$-prime. Indeed, for $\left|a_{i}\right|_{\eta}<1$, we have

$$
\left.b \circ\left(\operatorname{id}_{Z} \oplus \bigoplus_{i} a_{i}\right) \circ b^{\prime}\right|_{\eta}<1 \Leftrightarrow\left|b \circ\left(\operatorname{id}_{Z} \oplus 0\right) \circ b^{\prime}\right|_{\eta}<1 .
$$

Here we may assume that $\left\{a_{i}\right\}$ consists of one element $a, Z=[1]$, and that $b={\overline{\left(b^{\prime}\right.}}^{\mathrm{t}}=\left(b_{0}, b_{1}\right)$ is a vector of norm 1, so (4.21.1) reads : for $|a|_{\eta}<1,\left|b_{0}\right|_{\eta}^{2}+\left|b_{1}\right|_{\eta}^{2}=1$,

$$
\left.|| b_{0}\right|_{\eta} ^{2}+\left.a \cdot\left|b_{1}\right|_{\eta}^{2}\right|_{\eta}<1 \quad \Leftrightarrow \quad\left|b_{0}^{2}\right|_{\eta}<1 .
$$

Verifying this (obvious) statement shows that $\mathfrak{m}_{\eta}$ is indeed an $E$-prime.

For $a \in\left(\mathcal{O}_{\mathbb{k}, \eta}\right)_{Y, X}$ we have the non-negative self-adjoint operators $\bar{a}^{\mathrm{t}} \circ a \in\left(\mathcal{O}_{\mathbb{k}, \eta}\right)_{X, X}$, and $a \circ \bar{a}^{\mathrm{t}} \in\left(\mathcal{O}_{\mathbb{k}, \eta}\right)_{Y, Y}$. We get orthogonal decompositions

$$
\begin{aligned}
& \mathbb{k} \cdot X=\bigoplus_{\lambda} V(\lambda), \\
& \mathbb{k} \cdot Y=\bigoplus_{\lambda} W(\lambda),
\end{aligned}
$$

where $\bar{a}^{\mathrm{t}} \circ a$ (respectively $a \circ \bar{a}^{\mathrm{t}}$ ) acts on $V(\lambda)$ (respectively $W(\lambda)$ ) as scalar multiplication by $\lambda^{2}, 0 \leqslant \lambda \leqslant 1$. For $\lambda>0, a$ induces a linear isomorphism

$$
a_{\lambda}: V(\lambda) \stackrel{\sim}{\longrightarrow} W(\lambda)
$$

and for $\lambda=1, a_{1}$ is an isometry. This gives the singular eigenvalue decomposition of $a$,

$$
a=b \circ\left(\bigoplus \lambda \cdot \mathrm{id}_{Z_{\lambda}}\right) \circ b^{\prime},
$$

where $Z_{\lambda}$ is an orthonormal basis for $V(\lambda), b^{\prime}$ is the change-of-basis matrix from $X$ to $\left\{Z_{\lambda}\right\}$, and $b$ is the change-of-basis matrix from $\left\{(1 / \lambda) \cdot a\left(Z_{\lambda}\right)\right\}$ to $Y$; thus $b, b^{\prime}$ are isomorphisms in $\mathcal{O}_{\mathbb{k}, \eta}$ (i.e. orthogonal or unitary matrices). In particular, we see that modulo $E \mathfrak{m}_{\eta}, a$ is equivalent to

$$
a_{1}=b \circ\left(\operatorname{id}_{Z_{1}} \oplus \bigoplus_{\lambda<1} 0_{Z_{\lambda}}\right) \circ b^{\prime} .
$$

It follows that the map $\pi(a)=\left(a_{1}: V(1) \rightarrow W(1)\right)$ is an isomorphism of $\mathcal{O}_{\mathbb{k}, \eta} / \mathfrak{m}_{\eta}$ onto $\mathbb{F}_{\mathbb{k}, \eta}$, the field of partial isometries of (2.18).

The last two examples give 'strong- $E$-primes' according to the following definition.

Definition 4.22. We define $E$ - $\operatorname{SPEC}(A)=\operatorname{SPEC}(A) \cap E$-sub $A(A)$; its elements will be called strong-E-primes, or $S$-E-primes. Every $\mathbb{F}$-ring contains a maximal (proper) $E$-ideal, and such an ideal is $S$-E-prime. More generally, given $\mathfrak{a} \in E$-sub $A(A), f \in A_{Y, X}$ such that $f^{\otimes n} \notin \mathfrak{a}$ for all $n$, there exists by Zorn's lemma a maximal element $\mathfrak{m}$ in the set

$$
\left\{\mathfrak{b} \in E-\operatorname{sub}_{A}(A) \mid \mathfrak{b} \supseteq \mathfrak{a}, f^{\otimes n} \notin \mathfrak{b} \forall n\right\},
$$

and such an $\mathfrak{m}$ is $S$-E-prime; cf. Claim 4.19.2. It follows that for an $E$-ideal $\mathfrak{a} \in E$-sub $A(A)$

$$
\sqrt{\mathfrak{a}}=\left\{f \in A \mid f^{\otimes n} \in \mathfrak{a} \text { for some } n \geqslant 1\right\}=\bigcap_{\mathfrak{a} \subseteq \mathfrak{p}} \mathfrak{p},
$$

the intersection of all $S$-E-primes $\mathfrak{p}$ containing $\mathfrak{a}$. 


\section{J. SHAi HARAN}

Thus we have four functors from $\mathbb{F}$-rings to compact sober topological spaces, taking an $\mathbb{F}$-ring $A$ to the following diagram.

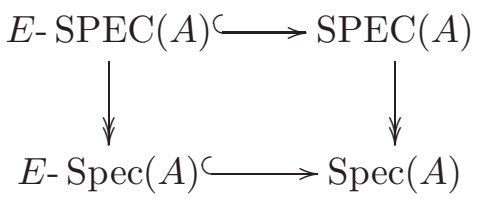

There are corresponding various notations of 'fields' for $\mathbb{F}$-rings.

Definition 4.23. An $\mathbb{F}$-ring $A$ is called a field, or an $H$-field, if it satisfies the equivalent conditions:

(i) $\operatorname{Spec}(A)=\{(0)\}$;

(ii) there are no non-zero proper $H$-ideals $\mathfrak{a} \subseteq A_{[1],[1]}$;

(iii) $G L_{[1]}(A)=A_{[1],[1]} \backslash\{0\}$ : every non-zero element of $A_{[1],[1]}$ is invertible.

An $\mathbb{F}$-ring $A$ is called an E-field, if it satisfies the equivalent conditions:

(i) $E-\operatorname{Spec}(A)=\{(0)\}$;

(ii) there are no non-zero proper $H$-E-ideals $\mathfrak{a} \subseteq A_{[1],[1]}$;

(iii) every homomorphism $\varphi: A \rightarrow B$ with $B \neq 0$ and such that $\mathcal{K} \mathcal{E R}(\varphi)=E\left(\varphi^{-1}(0)_{[1],[1]}\right)$ is injective, i.e., $\mathcal{K} \mathcal{E R}(\varphi)$ is trivial (= diagonal of $A \prod A$; cf., $(2.20)$ for $\mathcal{K} \mathcal{E} \mathcal{R}(\varphi)$ ).

An $\mathbb{F}$-ring $A$ is called a strong field or an $S$-field, if it satisfies the equivalent conditions:

(i) $\operatorname{SPEC}(A)=\{(0)\}$;

(ii) there are no non-zero proper ideals $\mathfrak{a} \subseteq A$;

(iii) for all $X, Y \in|\mathbb{F}|$, every non-zero element of $A_{Y, X}$ is a unit.

An $\mathbb{F}$-ring $A$ is called a strong-E-field or an $S$-E-field, if it satisfies the equivalent conditions:

(i) $E-\operatorname{SPEC}(A)=\{(0)\}$;

(ii) there are no non-zero proper $E$-ideals $\mathfrak{a}$;

(iii) every homomorphism $\varphi: A \rightarrow B$ with $B \neq 0$ and such that $\mathcal{K} \mathcal{E} \mathcal{R}(\varphi)=E\left(\varphi^{-1}(0)\right)$ is injective.

We have the following implications:

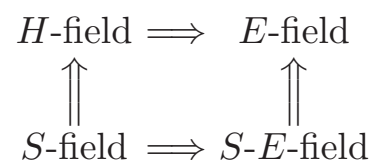

In particular, if $\mathfrak{m}$ is a maximal $H$-ideal of an $\mathbb{F}$-ring $\mathcal{O}$, we have the residue field $\mathbb{F}_{\mathfrak{m}}=\mathcal{O} / E \mathfrak{m}$; it is an $H$-field by (4.3): if $\mathfrak{n}$ is an $H$-ideal of $\mathcal{O}$, and $\mathfrak{n}$ is $E \mathfrak{m}$-stable, (4.3), then $\mathfrak{n}$ contains $\mathfrak{m}$, and by maximality $\mathfrak{n}=\mathfrak{m}$.

\section{Localization and structural sheaf $\mathcal{O}_{A}$}

We review the theory of localization of an $\mathbb{F}$-ring $A$ (or an $A$-module $M$ ) with respect to a multiplicative set $S \subseteq A_{[1],[1]}$; we obtain a sheaf of $\mathbb{F}$-rings $\mathcal{O}_{A}$ (respectively, an $\mathcal{O}_{A}$-module $\widetilde{M}$ ) over $\operatorname{Spec} A$.

\subsection{Localization}

The theory of localization of an $\mathbb{F}$-ring $A$, with respect to a multiplicative subset $S \subseteq A_{[1],[1]}$, goes exactly as in localization of commutative rings - since it is a multiplicative theory. We recall this theory next. 


\section{NON-ADDITIVE GEOMETRY}

We assume that $S \subseteq A_{[1],[1]}$ satisfies

$$
\begin{gathered}
1 \in S, \\
s_{1}, s_{2} \in S \quad \Rightarrow \quad s_{1} \cdot s_{2} \in S .
\end{gathered}
$$

On the set

$$
A \times S=\coprod_{Y, X} A_{Y, X} \times S
$$

we define for $a_{i} \in A_{Y, X}, s_{i} \in S$,

$$
\left(a_{1}, s_{1}\right) \sim\left(a_{2}, s_{2}\right) \Leftrightarrow s \cdot s_{2} \cdot a_{1}=s \cdot s_{1} \cdot a_{2} \text { for some } s \in S .
$$

It follows that $\sim$ is an equivalence relation, and we denote by $a / s$ the equivalence class containing $(a, s)$, and by $S^{-1} A$ the collection of equivalence classes. On $S^{-1} A$ we define the operations:

$$
\begin{gathered}
a_{1} / s_{1} \circ a_{2} / s_{2}=\left(a_{1} \circ a_{2}\right) / s_{1} s_{2}, \quad a_{1} \in A_{Z, Y}, a_{2} \in A_{Y, X}, \\
a_{1} / s_{1} \oplus a_{2} / s_{2}=\left(s_{2} \cdot a_{1} \oplus s_{1} \cdot a_{2}\right) / s_{1} s_{2}, \\
a_{1} / s_{1} \otimes a_{2} / s_{2}=\left(a_{1} \otimes a_{2}\right) / s_{1} s_{2} .
\end{gathered}
$$

Proposition 5.3. The above operations are well defined, independent of the chosen representatives, and they satisfy the axioms of an $\mathbb{F}$-ring.

Proof. The usual proof works. For example, replacing $a_{1} / s_{1}$ in (5.3.2) by $a_{1}^{\prime} / s_{1}^{\prime} \sim a_{1} / s_{1}$, say $s \cdot s_{1}^{\prime}$. $a_{1}=s \cdot s_{1} \cdot a_{1}^{\prime}$, then

$$
s \cdot s_{1}^{\prime} s_{2} \cdot\left(s_{2} a_{1} \oplus s_{1} a_{2}\right)=s \cdot s_{1} s_{2} \cdot\left(s_{2} a_{1}^{\prime} \oplus s_{1}^{\prime} a_{2}\right),
$$

hence

$$
\left(s_{2} a_{1} \oplus s_{1} a_{2}\right) / s_{1} s_{2}=\left(s_{2} a_{1}^{\prime} \oplus s_{1}^{\prime} a_{2}\right) / s_{1}^{\prime} s_{2} .
$$

The $\mathbb{F}$-ring $S^{-1} A$ comes with a canonical homomorphism

$$
\phi=\phi_{S}: A \rightarrow S^{-1} A, \quad \phi(a)=a / 1 .
$$

Proposition 5.5. We have the universal property of $\phi_{S}$ :

$$
\begin{gathered}
\operatorname{Hom}_{\mathbb{F}-\mathcal{R} \text { ings }}\left(S^{-1} A, B\right)=\left\{\varphi \in \operatorname{Hom}_{\mathbb{F}-\mathcal{R} \text { ings }}(A, B) \mid \varphi(S) \subseteq G L_{[1]}(B)\right\}, \\
\widetilde{\varphi} \longmapsto \widetilde{\varphi} \circ \phi_{S}, \\
\widetilde{\varphi}(a / s)=\varphi(a) \cdot \varphi(s)^{-1} \longleftrightarrow \varphi .
\end{gathered}
$$

Proof. The proof is clear.

Note that $S^{-1} A$ is the zero $\mathbb{F}$-ring if and only if $0 \in S$.

The main examples of localizations are as follows:

$$
S_{f}=\left\{f^{n}\right\}_{n \geqslant 0}, \quad f \in A_{[1],[1]},
$$

and we write $A_{f}$ for $S_{f}^{-1} A$;

and we write $A_{\mathfrak{p}}$ for $S_{\mathfrak{p}}^{-1} A$.

$$
S_{\mathfrak{p}}=A_{[1],[1]} \backslash \mathfrak{p}, \quad \mathfrak{p} \in \operatorname{Spec}(A),
$$

Similarly, for an $A$-module $M$, we have the equivalence relation $\sim$ on $M \times S$,

$$
\left(m_{1}, s_{1}\right) \sim\left(m_{2}, s_{2}\right) \Leftrightarrow s \cdot s_{2} \cdot m_{1}=s \cdot s_{1} \cdot m_{2} \quad \text { for some } s \in S .
$$

We let $m / s=(m, s) / \sim$ denote the equivalence class containing $(m, s)$, and $S^{-1} M=(M \times S) / \sim$ denote the collection of equivalence classes. On $S^{-1} M$ we have the structure of an $S^{-1} A$-module, 


\section{J. SHAi HARAN}

which is well defined, independent of the chosen representatives, by

$$
\begin{gathered}
a_{1} / s_{1} \circ m / s \circ a_{2} / s_{2}=\left(a_{1} \circ m \circ a_{2}\right) / s_{1} s s_{2}, \\
m_{1} / s_{1} \oplus m_{2} / s_{2}=\left(s_{2} \cdot m_{1} \oplus s_{1} \cdot m_{2}\right) / s_{1} s_{2}, \\
a_{1} / s_{1} \otimes m / s=\left(a_{1} \otimes m\right) / s_{1} s .
\end{gathered}
$$

The localization $M \mapsto S^{-1} M$ is a functor $S^{-1}: A-\mathcal{M}$ od $\rightarrow S^{-1} A-\mathcal{M}$ od; to a map $\varphi: M \rightarrow M^{\prime}$ corresponds $S^{-1} \varphi: S^{-1} M \rightarrow S^{-1} M^{\prime}$,

$$
S^{-1} \varphi(m / s)=\varphi(m) / s .
$$

We have an $A$-module homomorphism $M \rightarrow \phi_{S}^{*}\left(S^{-1} M\right), m \mapsto m / 1$, which corresponds by (3.23.6) to the homomorphism of $S^{-1} A$ modules

$$
M_{S^{-1} A} \rightarrow S^{-1} M, \quad\left(a_{1} / s_{1}, m, a_{2} / s_{2}\right) / \sim \mapsto\left(a_{1} \circ m \circ a_{2}\right) / s_{1} s_{2},
$$

where $M_{S^{-1} A}$ is the $S^{-1} A$-module obtained from $M$ via base change (3.23.0) along the homomorphism $A \rightarrow S^{-1} A$. This is clearly surjective. It is also injective. Note first that by (3.23.1), (3.23.2), $\left(a_{1} / s_{1}, m, a_{2} / s_{2}\right) / \sim=\left(\mathrm{id} / 1, a_{1} \circ m \circ a_{2}, \mathrm{id} / s_{1} s_{2}\right) / \sim$, so every element of $M_{S^{-1} A}$ has the form $(\mathrm{id} / 1, m, \mathrm{id} / s) / \sim$. If $m_{1} / s_{1}=m_{2} / s_{2}$, say $s \cdot s_{2} \cdot m_{1}=s \cdot s_{1} \cdot m_{2}$, then we have the following:

$$
\begin{aligned}
\left(\mathrm{id} / 1, m_{1}, \mathrm{id} / s_{1}\right) / \sim & =\left(\mathrm{id} / 1, m_{1}, s \cdot s_{2} / 1 \cdot \mathrm{id} / s s_{1} s_{2}\right) / \sim=\left(\mathrm{id} / 1, s \cdot s_{2} \cdot m_{1}, \mathrm{id} / s s_{1} s_{2}\right) / \sim \\
\left(\mathrm{id} / 1, m_{2}, \mathrm{id} / s_{2}\right) / \sim & =\left(\mathrm{id} / 1, m_{2}, s \cdot s_{1} / 1 \cdot \mathrm{id} / s s_{1} s_{2}\right) / \sim=\left(\mathrm{id} / 1, s \cdot s_{1} \cdot m_{2}, \mathrm{id} / s s_{1} s_{2}\right) / \sim
\end{aligned}
$$

Thus we may identify the localization $S^{-1} M$ with the base change $M_{S^{-1}}$, and it follows from (3.23.7), (3.23.8), (3.23.9) that we have similar properties for localization.

Corollary 5.7.6. The functor $M \mapsto S^{-1} M$ preserves sums, direct limits, tensor products, and free modules:

$$
\begin{gathered}
S^{-1} \coprod_{i} M_{i}=\coprod_{i} S^{-1} M_{i}, \quad S^{-1} \lim _{\longrightarrow} M_{\lambda}=\underline{\lim }_{\longrightarrow} S^{-1} M_{\lambda} \\
S^{-1}\left(M \otimes_{A} N\right)=S^{-1} M \otimes_{S^{-1} A} S^{-1} N
\end{gathered}
$$

for a formal symbol $f_{X, Y}$, any $Y, X \in|\mathbb{F}|$, and for the free $A$-module $A \cdot f_{Y, X}$, and the free $S^{-1} A$-module $\left(S^{-1} A\right) \cdot f_{Y, X}$, cf. (3.18.0),

$$
S^{-1}\left(A \cdot f_{Y, X}\right)=\left(S^{-1} A\right) \cdot f_{Y, X}
$$

If $M_{0} \in \operatorname{sub}_{A}(M)$ is an $A$-submodule, then $S^{-1} M_{0} \in \operatorname{sub}_{S^{-1} A}\left(S^{-1} M\right)$ is an $S^{-1} A$-submodule. The map $M_{0} \mapsto S^{-1} M_{0}$ preserves sums,

$$
S^{-1}\left(\sum_{i} M_{i}\right)=\sum_{i}\left(S^{-1} M_{i}\right)
$$

and finite intersections,

$$
S^{-1}\left(M_{1} \cap M_{2}\right)=S^{-1} M_{1} \cap S^{-1} M_{2} .
$$

Since we can always bring a finite sequence of elements in $S$-localization into 'common denominator', we have the following result.

Proposition 5.9. The functor $M \mapsto S^{-1} M$ also preserves finite products, and finite inverse limits,

$$
S^{-1}\left(M_{0} \prod_{M} M_{1}\right)=S^{-1} M_{0} \prod_{S^{-1} M} S^{-1} M_{1}, \quad\left(m_{0}, m_{1}\right) / s \mapsto\left(m_{0} / s, m_{1} / s\right) .
$$




\section{NON-ADDITIVE GEOMETRY}

If $\mathcal{E} \in \operatorname{equiv}_{A}(M)$ is an equivalence $A$-module of $M$, then

$$
S^{-1} \mathcal{E} \subseteq S^{-1}\left(M \prod M\right)=S^{-1} M \prod S^{-1} M
$$

is an equivalence $S^{-1} A$-module of $S^{-1} M, S^{-1} \mathcal{E} \in$ equiv $_{S^{-1} A}\left(S^{-1} M\right)$. We have (with $Z, E$ as in Definition 3.10)

$$
S^{-1}(Z \mathcal{E})=Z\left(S^{-1} \mathcal{E}\right), \quad \mathcal{E} \in \operatorname{equiv}_{A}(M)
$$

and

$$
S^{-1}\left(E M_{0}\right)=E\left(S^{-1} M_{0}\right), \quad M_{0} \in \operatorname{sub}_{A}(M)
$$

(cf., Lemma 3.13: bring a path to a common denominator).

Similarly we have the next proposition.

Proposition 5.11. Let $\varphi: M \rightarrow N$ be a homomorphism of $A$-modules. The functor $S^{-1}$ preserves kernels,

$$
\begin{aligned}
S^{-1}\left(\varphi^{-1}(0)\right) & =\left(S^{-1} \varphi\right)^{-1}(0), \\
S^{-1}(\mathcal{K} \mathcal{E} \mathcal{R}(\varphi)) & =\mathcal{K} \mathcal{E} \mathcal{R}\left(S^{-1} \varphi\right),
\end{aligned}
$$

and it preserves cokernels,

$$
S^{-1}(N / \varphi(M))=S^{-1} N / S^{-1} \varphi\left(S^{-1} M\right) .
$$

Definition 5.11.4. We write $M_{\mathfrak{p}}$ for $S_{\mathfrak{p}}^{-1} M, S_{\mathfrak{p}}=A_{[1],[1]} \backslash \mathfrak{p}, \mathfrak{p} \in \operatorname{Spec} A$. We write $M_{f}$ for $S_{f}^{-1} M$, $S_{f}=\left\{f^{n}\right\}_{n \geqslant 0}, f \in A_{[1],[1]}$.

Proposition 5.12. For an $A$-module $M$, the following are equivalent:

$$
\begin{gathered}
M=0, \\
M_{\mathfrak{p}}=0 \forall \mathfrak{p} \in \operatorname{Spec} A, \\
M_{\mathfrak{m}}=0 \text { for all maximal } H \text {-ideals } \mathfrak{m} .
\end{gathered}
$$

Proof. Clearly $(5.12 .1) \Rightarrow(5.12 .2) \Rightarrow(5.12 .3)$. If $M \neq 0$ let $m \in M_{Y, X}$ be non-zero, and let $\mathfrak{m}$ be a maximal $H$-ideal containing $\operatorname{ann}_{A}(m)$; cf. (4.1.4). If $M_{\mathfrak{m}}=0 \Rightarrow m / 1=0 \in M_{\mathfrak{m}}$, which means $s \cdot m=0$ for some $s \in A_{[1],[1]} \backslash \mathfrak{m}$, contradicting $\operatorname{ann}_{A}(m) \subseteq \mathfrak{m}$.

Proposition 5.13. Let $\varphi: M \rightarrow N$ be a homomorphism of $A$-modules. The following are equivalent:

$$
\begin{gathered}
\varphi \text { is surjective, } \\
\varphi_{\mathfrak{p}}: M_{\mathfrak{p}} \rightarrow N_{\mathfrak{p}} \text { is surjective } \forall \mathfrak{p} \in \operatorname{Spec} A, \\
\varphi_{\mathfrak{m}}: M_{\mathfrak{m}} \rightarrow N_{\mathfrak{m}} \text { is surjective for all maximal } H \text {-ideals } \mathfrak{m} .
\end{gathered}
$$

Proof. To show that $(5.13 .1) \Rightarrow(5.13 .2) \Rightarrow(5.13 .3)$ is easy. If $\varphi$ is not surjective, let $n \in N \backslash \varphi(M)$, and let $\mathfrak{m}$ be a maximal $H$-ideal containing $(\varphi(M): n)$; cf. (4.1.6). If $\varphi_{\mathfrak{m}}$ is surjective, $n / 1 \in$ $\varphi_{\mathfrak{m}}\left(M_{\mathfrak{m}}\right)$, and we have $s \cdot n \in \varphi(M)$ for some $s \in A_{[1],[1]} \backslash \mathfrak{m}$, contradicting $(\varphi(M): n) \subseteq \mathfrak{m}$.

Proposition 5.14. Let $\varphi: M \rightarrow N$ be a homomorphism of $A$-modules. The following are equivalent:

$$
\begin{gathered}
\varphi \text { is injective, } \\
\varphi_{\mathfrak{p}}: M_{\mathfrak{p}} \rightarrow N_{\mathfrak{p}} \text { is injective } \forall \mathfrak{p} \in \operatorname{Spec} A, \\
\varphi_{\mathfrak{m}}: M_{\mathfrak{m}} \rightarrow N_{\mathfrak{m}} \text { is injective for all maximal } H \text {-ideals } \mathfrak{m} .
\end{gathered}
$$




\section{J. SHAI HARAN}

Proof. To show that $(5.14 .1) \Rightarrow(5.14 .2) \Rightarrow(5.14 .3)$ is easy. If $\varphi$ is not injective, let $m \neq m^{\prime}, \varphi(m)=$ $\varphi\left(m^{\prime}\right)$, and let $\mathfrak{m}$ be a maximal $H$-ideal containing $a n n_{A}\left(m, m^{\prime}\right)$; cf. (4.1.5). If $\varphi_{\mathfrak{m}}$ is injective, $m / 1=m^{\prime} / 1 \in M_{\mathfrak{m}}$, and $s \cdot m=s \cdot m^{\prime}$ for some $s \in A_{[1],[1]} \backslash \mathfrak{m}$, contradicting $\operatorname{ann}_{A}\left(m, m^{\prime}\right) \subseteq \mathfrak{m}$.

Consider the canonical homomorphism $\phi=\phi_{S}: A \rightarrow S^{-1} A, \phi(a)=a / 1$. If $\mathfrak{b} \in \operatorname{sub}_{S^{-1} A}\left(S^{-1} A\right)$ is an ideal of $S^{-1} A, \phi^{-1}(\mathfrak{b}) \in \operatorname{sub}_{A}(A)$ is an ideal of $A$; if $\mathfrak{b}=Z E(\mathfrak{b})$ is an $E$-ideal, so is $\phi^{-1}(\mathfrak{b})$, cf. (4.16.2) for $Z E(\mathfrak{b})$; if $\mathfrak{b}$ is homogeneous so is $\phi^{-1}(\mathfrak{b})$. If $\mathfrak{b} \in H$-id $\left(S^{-1} A\right)$ is an $H$-ideal, so is $\varphi^{-1}(\mathfrak{b})$. If $\mathfrak{a} \in \operatorname{sub}_{A}(A)$ is an ideal of $A$, then $\phi(\mathfrak{a})$ generates the ideal $S^{-1} \mathfrak{a} \in \operatorname{sub}_{S^{-1}}\left(S^{-1} A\right)$; if $\mathfrak{a}=Z E(\mathfrak{a})$ is an $E$-ideal, so is $S^{-1} \mathfrak{a}$; if $\mathfrak{a}$ is homogeneous, so is $S^{-1} \mathfrak{a}$. If $\mathfrak{A} \in H$-id( $\left.A\right)$ is an $H$-ideal of $A$ then

$$
S^{-1} \mathfrak{A} \stackrel{\text { def }}{=} \phi_{*}(\mathfrak{A})=\left\{a / s \in\left(S^{-1} A\right)_{[1],[1]} \mid a \in \mathfrak{A}, s \in S\right\}
$$

is an $H$-ideal of $S^{-1} A$.

Proposition 5.15. For $\mathfrak{b}$ an ideal (respectively $H$-ideal) of $S^{-1} A, S^{-1} \mathfrak{b}^{c}=\mathfrak{b}$.

Proof. If $a / s \in \mathfrak{b}, a \in \mathfrak{b}^{c}$, and $a / s \in S^{-1}\left(\mathfrak{b}^{c}\right)$; so $\mathfrak{b} \subseteq S^{-1}\left(\mathfrak{b}^{c}\right)$. The reverse inclusion is clear.

Proposition 5.16. For $\mathfrak{a}$ an ideal (respectively $H$-ideal) of $A$,

$$
\left(S^{-1} \mathfrak{a}\right)^{c}=\{a \in A \mid \exists s \in S: s \cdot a \in \mathfrak{a}\} .
$$

In particular,

$$
S^{-1} \mathfrak{a}=(1) \quad \Leftrightarrow \quad \mathfrak{a} \cap S \neq \varnothing
$$

Proof. We have

$$
a \in\left(S^{-1} \mathfrak{a}\right)^{c} \quad \Leftrightarrow \quad a / 1=x / s, x \in \mathfrak{a}, s \in S \quad \Leftrightarrow \quad s \cdot a \in \mathfrak{a}, \text { some } s \in S .
$$

Proposition 5.17. The map $\phi_{S}^{*}$ induces a bijection

$$
\phi_{S}^{*}: \operatorname{Spec}\left(S^{-1} A\right) \stackrel{\sim}{\longrightarrow}\{\mathfrak{p} \in \operatorname{Spec} A \mid \mathfrak{p} \cap S=\varnothing\},
$$

which is a homeomorphism for the Zariski topology.

Proof. If $\mathfrak{q} \in \operatorname{Spec}\left(S^{-1} A\right), \phi_{S}^{*}(\mathfrak{q})$ belongs to the right-hand side. Conversely, if $\mathfrak{p}$ belongs to the right-hand side, $S^{-1} \mathfrak{p}$ is a (proper) prime of $S^{-1} A$. By Propositions 5.15 and 5.16 these operations are inverses of each other.

Corollary 5.17.1. We have a homeomorphism for $f \in A_{[1],[1]}$,

$$
\phi_{f}^{*}: \operatorname{Spec}\left(A_{f}\right) \stackrel{\sim}{\longrightarrow} D_{A}(f) .
$$

Corollary 5.17.2. We have a homeomorphism for $\mathfrak{p} \in \operatorname{Spec}(A)$,

$$
\phi_{\mathfrak{p}}^{*}: \operatorname{Spec}\left(A_{\mathfrak{p}}\right) \stackrel{\sim}{\longrightarrow}\{\mathfrak{q} \in \operatorname{Spec} A \mid \mathfrak{q} \subseteq \mathfrak{p}\} .
$$

In particular, $A_{\mathfrak{p}}$ contains a unique maximal $H$-ideal $\mathfrak{m}_{\mathfrak{p}}=S_{\mathfrak{p}}^{-1} \mathfrak{p}$; we say it is a local $\mathbb{F}$-ring.

Remark 5.17.3. For $\mathfrak{p} \in \operatorname{Spec}(A)$ we let $\mathbb{F}_{\mathfrak{p}}=A_{\mathfrak{p}} / \mathfrak{m}_{\mathfrak{p}}$ denote the residue field at $\mathfrak{p}$. Let $\pi: A \rightarrow A / \mathfrak{p}$ be the canonical homomorphism, and $\bar{S}_{\mathfrak{p}}=\pi\left(S_{\mathfrak{p}}\right)$; we have also $\mathbb{F}_{\mathfrak{p}}=\bar{S}_{\mathfrak{p}}^{-1}(A / \mathfrak{p})$. The commutative diagram

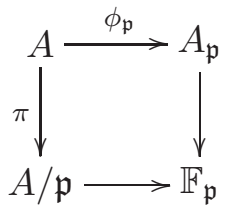


is cartesian: $\mathbb{F}_{\mathfrak{p}}=(A / \mathfrak{p}) \odot_{A} A_{\mathfrak{p}}$ (see Theorem 7.1 for the definition of $\odot$ ). It is also functorial: given a homomorphism of $\mathbb{F}$-rings $\varphi: A \rightarrow B, \mathfrak{q} \in \operatorname{Spec} B, \mathfrak{p}=\varphi^{*}(\mathfrak{q})$, we have the following commutative cube:

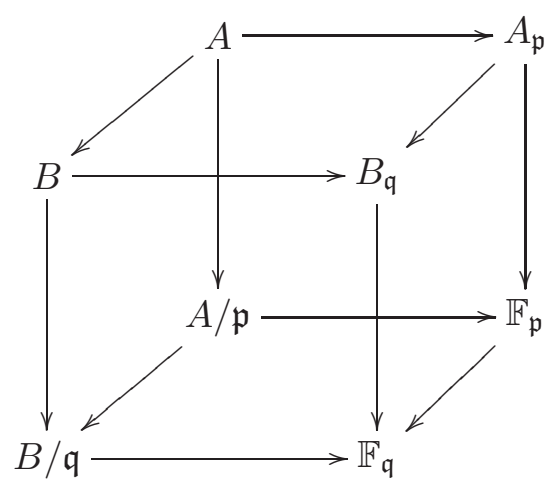

\subsection{Structural sheaf $\mathcal{O}_{A}$}

Next we define a sheaf $\mathcal{O}_{A}$ of $\mathbb{F}$-rings over $\operatorname{Spec} A$.

Definition 5.18. For an open set $U \subseteq \operatorname{Spec}(A)$, and for $Y, X \in|\mathbb{F}|$, we let $\mathcal{O}_{A}(U)_{Y, X}$ denote the set of functions

$$
s: U \rightarrow \bigcup_{\mathfrak{p} \in U}\left(A_{\mathfrak{p}}\right)_{Y, X},
$$

such that $s(\mathfrak{p}) \in\left(A_{\mathfrak{p}}\right)_{Y, X}$, and $s$ is 'locally a fraction':

$$
\forall \mathfrak{p} \in U, \exists \text { a neighborhood } U_{\mathfrak{p}} \text { of } \mathfrak{p} ; \exists a \in A_{Y, X} ; \exists f \in A_{[1],[1]} \backslash \bigcup_{\mathfrak{q} \in U_{\mathfrak{p}}} \mathfrak{q}
$$

such that

$$
s(\mathfrak{q})=a / f \in A_{\mathfrak{q}}, \quad \forall \mathfrak{q} \in U_{\mathfrak{p}}
$$

It is clear that

$$
\mathcal{O}_{A}(U)=\bigcup_{Y, X} \mathcal{O}_{A}(U)_{Y, X}
$$

is an $\mathbb{F}$-ring. If $U^{\prime} \subseteq U$, the natural restriction map $\left.s \mapsto s\right|_{U^{\prime}}$ is a homomorphism of $\mathbb{F}$-rings $\mathcal{O}_{A}(U) \rightarrow$ $\mathcal{O}_{A}\left(U^{\prime}\right)$, thus $\mathcal{O}_{A}$ is a presheaf of $\mathbb{F}$-rings. From the local nature of $(\star)$ we see that $\mathcal{O}_{A}$ is in fact a sheaf.

Remark 5.18.1. Similarly for an $A$-module $M$ we can define $\widetilde{M}(U)_{Y, X}$ as the collection of sections

$$
s: U \rightarrow \bigcup_{\mathfrak{p} \in U}\left(M_{\mathfrak{p}}\right)_{Y, X}
$$

which are locally a fraction (replace $a \in A_{Y, X}$ in $(\star)$ by $\mathfrak{m} \in M_{Y, X}$ ). The set $\widetilde{M}$ is an $\mathcal{O}_{A}$-module in the following sense.

Definition 5.18.2. An $\mathcal{O}_{A}$-module $\mathfrak{M}$ is a sheaf of sets over $\operatorname{Spec} A$ such that $\mathfrak{M}(U)$ is an $\mathcal{O}_{A}(U)$ module, the structure compatible with restrictions - for open sets $U \subseteq U^{\prime}$, denoting by $\left.s \mapsto s\right|_{U}$ the restriction maps $\mathfrak{M}\left(U^{\prime}\right) \rightarrow \mathfrak{M}(U)$ and $\mathcal{O}_{A}\left(U^{\prime}\right) \rightarrow \mathcal{O}_{A}(U)$, we have

$$
\begin{gathered}
\left.\left(a \circ m \circ a^{\prime}\right)\right|_{U}=\left.\left.\left.a\right|_{U} \circ m\right|_{U} \circ a^{\prime}\right|_{U}, \\
\left.\left(m \oplus m^{\prime}\right)\right|_{U}=\left.\left.m\right|_{U} \oplus m^{\prime}\right|_{U}, \\
\left.(a \otimes m)\right|_{U}=\left.\left.a\right|_{U} \otimes m\right|_{U} .
\end{gathered}
$$




\section{J. Shai Haran}

For two such sheaves of $\mathcal{O}_{A}$-modules $\mathfrak{M}, \mathfrak{M}^{\prime}$ a map of $\mathcal{O}_{A}$-modules $\varphi: \mathfrak{M} \rightarrow \mathfrak{M}^{\prime}$ is a collection of $\mathcal{O}_{A}(U)$-homomorphisms

$$
\varphi_{U}: \mathfrak{M}(U) \rightarrow \mathfrak{M}^{\prime}(U)
$$

for $U \subseteq \operatorname{Spec} A$ open, compatible with restrictions: $\left.\varphi_{U^{\prime}}(a)\right|_{U}=\varphi_{U}\left(\left.a\right|_{U}\right)$ for $U \subseteq U^{\prime}$.

Thus we have the category $\mathcal{O}_{A}$ - Mod of $\mathcal{O}_{A}$-modules.

Proposition 5.19. For $\mathfrak{p} \in \operatorname{Spec}(A)$, the stalk

$$
\mathcal{O}_{A, \mathfrak{p}}=\underset{\mathfrak{p} \in U}{\lim _{A}} \mathcal{O}_{A}(U)
$$

of the sheaf $\mathcal{O}_{A}$ is isomorphic to $A_{\mathfrak{p}}$.

Proof. The map taking a local section $s$ in a neighborhood of $\mathfrak{p}$ to $s(\mathfrak{p}) \in A_{\mathfrak{p}}$ induces a homomorphism $\mathcal{O}_{A, \mathfrak{p}} \rightarrow A_{\mathfrak{p}}$, which is clearly surjective. It is also injective. Let $s_{1}, s_{2} \in \mathcal{O}_{A}(U)_{Y, X}$ have the same value at $\mathfrak{p}, s_{1}(\mathfrak{p})=s_{2}(\mathfrak{p})$. Shrinking $U$ we may assume $s_{i}=a_{i} / f_{i}$ on $U, a_{i} \in A_{Y, X}, f_{i} \in A_{[1],[1]}$. Then $a_{1} / f_{1}=a_{2} / f_{2}$ in $A_{\mathfrak{p}}$ means $h \cdot f_{2} \cdot a_{1}=h \cdot f_{1} \cdot a_{2}, h \in A_{[1],[1]} \backslash \mathfrak{p}$, but then $a_{1} / f_{1}=a_{2} / f_{2}$ in $A_{\mathfrak{q}} \forall \mathfrak{q} \in U \cap D_{A}(h)$.

Remark 5.19.1. Similarly for an $A$-module $M$ we have an isomorphism

$$
(\widetilde{M})_{\mathfrak{p}} \stackrel{\text { def }}{=} \underset{\mathfrak{p} \in U}{\lim } \widetilde{M}(U) \stackrel{\sim}{\longrightarrow} M_{\mathfrak{p}} .
$$

Proposition 5.20. For $f \in A_{[1],[1]}$, the $\mathbb{F}$-ring $\mathcal{O}_{A}\left(D_{A}(f)\right)$ is isomorphic to $A_{f}$. In particular, the global sections $\Gamma\left(\operatorname{Spec}(A), \mathcal{O}_{A}\right) \stackrel{\text { def }}{=} \mathcal{O}_{A}\left(D_{A}(1)\right) \cong A$.

Proof. Define the homomorphism $\psi: A_{f} \rightarrow \mathcal{O}_{A}\left(D_{A}(f)\right)$ by sending $a / f^{n}$ to the section whose value at $\mathfrak{p}$ is the image of $a / f^{n}$ in $A_{\mathfrak{p}}$.

We shall show that $\psi$ is injective. If $\psi\left(a_{1} / f^{n_{1}}\right)=\psi\left(a_{2} / f^{n_{2}}\right)$ then $\forall \mathfrak{p} \in D_{A}(f)$ there is $h_{\mathfrak{p}} \in$ $A_{[1],[1]} \backslash \mathfrak{p}$ with

$$
h_{\mathfrak{p}} f^{n_{2}} a_{1}=h_{\mathfrak{p}} f^{n_{1}} a_{2} .
$$

Let $\mathfrak{a}=\operatorname{ann}_{A}\left(f^{n_{2}} a_{1}, f^{n_{1}} a_{2}\right)$. It is an $H$-ideal of $A$, and $\forall \mathfrak{p} \in D_{A}(f), \mathfrak{p} \notin V_{A}(\mathfrak{a})$, so $D_{A}(f) \cap V_{A}(\mathfrak{a})=\varnothing$, hence $V_{A}(\mathfrak{a}) \subseteq V_{A}(f)$, hence $f \in I V_{A}(\mathfrak{a})=\sqrt{\mathfrak{a}}$, hence $f^{n} \in \mathfrak{a}$ for some $n \geqslant 1$, showing that $a_{1} / f^{n_{1}}=a_{2} / f^{n_{2}}$ in $A_{f}$.

We show next that $\psi$ is surjective. Let $s \in \mathcal{O}_{A}\left(D_{A}(f)\right)_{Y, X}$. By Proposition $4.12, D_{A}(f)$ is compact, so there exists a finite open covering

$$
D_{A}(f)=\bigcup_{1 \leqslant i \leqslant N} D_{A}\left(h_{i}\right),
$$

such that for all $\mathfrak{p} \in D_{A}\left(h_{i}\right): s(\mathfrak{p})=a_{i} / g_{i} \in A_{\mathfrak{p}}$, where $a_{i} \in A_{Y, X}$ and $g_{i} \in A_{[1],[1]}$ is such that $D_{A}\left(g_{i}\right) \supseteq D_{A}\left(h_{i}\right)$ for $1 \leqslant i \leqslant N$. We have $V_{A}\left(g_{i}\right) \subseteq V_{A}\left(h_{i}\right)$, hence

$$
\sqrt{\left(g_{i}\right)}=I V_{A}\left(g_{i}\right) \supseteq I V_{A}\left(h_{i}\right)=\sqrt{\left(h_{i}\right)},
$$

hence $h_{i} \in \sqrt{\left(g_{i}\right)}$ so that for some $n_{i} \geqslant 1$ we have $h_{i}^{n_{i}}=c_{i} \cdot g_{i}$, hence $s(\mathfrak{p})=c_{i} a_{i} / h_{i}^{n_{i}}$. So we can replace $h_{i}$ by $g_{i}$. On the set

$$
D_{A}\left(g_{i}\right) \cap D_{A}\left(g_{j}\right)=D_{A}\left(g_{i} g_{j}\right)
$$

we have $a_{i} / g_{i}=s(\mathfrak{p})=a_{j} / g_{j}$, hence by the injectivity of $\psi$ we find

$$
a_{i} / g_{i}=a_{j} / g_{j} \text { in } A_{g_{i} g_{j}} \text {. }
$$

This means $\left(g_{i} g_{j}\right)^{n} \cdot g_{j} a_{i}=\left(g_{i} g_{j}\right)^{n} \cdot g_{i} a_{j}$, and we can choose $n$ big enough to work for all $i, j$. We can replace $g_{i}$ by $g_{i}^{n+1}\left(\right.$ since $\left.D_{A}\left(g_{i}\right)=D_{A}\left(g_{i}^{n+1}\right)\right)$, and replace $a_{i}$ by $g_{i}^{n} \cdot a_{i}\left(\right.$ since $\left.s(\mathfrak{p}) \equiv g_{i}^{n} a_{i} / g_{i}^{n+1}\right)$, 
and then have the simpler equation $g_{j} \cdot a_{i}=g_{i} \cdot a_{j}$ for all $i, j$. Since the sets $D_{A}\left(g_{i}\right)$ cover $D_{A}(f)$ we have, cf. Proposition 4.12,

$$
f^{m}=b \circ\left(\bigoplus_{i} g_{i} \cdot \mathrm{id}_{X_{i}}\right) \circ b^{\prime} .
$$

Set

$$
a=\left(\operatorname{id}_{Y} \otimes b\right) \circ\left(\bigoplus_{i} a_{i} \otimes \operatorname{id}_{X_{i}}\right) \circ\left(\operatorname{id}_{X} \otimes b^{\prime}\right) .
$$

Then

$$
\begin{aligned}
g_{j} \cdot a & =\left(\operatorname{id}_{Y} \otimes b\right) \circ\left(\bigoplus_{i} g_{j} a_{i} \otimes \operatorname{id}_{X_{i}}\right) \circ\left(\operatorname{id}_{X} \otimes b^{\prime}\right) \\
& =\left(\operatorname{id}_{Y} \otimes b\right) \circ\left(\bigoplus_{i} a_{j} \otimes g_{i} \cdot \mathrm{id}_{X_{i}}\right) \circ\left(\operatorname{id}_{X} \otimes b^{\prime}\right)=f^{m} \cdot a_{j} .
\end{aligned}
$$

Hence $a_{j} / g_{j}=s(\mathfrak{p})=a / f^{m}$ and $s=\psi\left(a / f^{m}\right)$.

Remark 5.20.1. Similarly for an $A$-module $M$ we have an isomorphism

$$
M_{f} \stackrel{\sim}{\longrightarrow} \widetilde{M}\left(D_{A}(f)\right)
$$

and in particular

$$
M \stackrel{\sim}{\rightarrow} \widetilde{M}(\operatorname{Spec} A) \stackrel{\text { def }}{=} \Gamma(\operatorname{Spec} A, \widetilde{M}) .
$$

\section{Schemes}

We define the categories of $\mathbb{F}$-(locally)-ringed spaces, and of (Zariski) $\mathbb{F}$-schemes, and recall the theory of quasi-coherent modules. As an important example we give the 'compactification' $\overline{\text { Spec } \mathbb{Z}}$ of $\operatorname{Spec} \mathbb{Z}$.

\subsection{Locally $\mathbb{F}$-ring spaces}

Definition 6.1. An $\mathbb{F}$-ringed space $\left(X, \mathcal{O}_{X}\right)$ is a topological space with a sheaf $\mathcal{O}_{X}$ of $\mathbb{F}$-rings. A map of $\mathbb{F}$-ringed spaces $f: X \rightarrow Y$ is a continuous map of the underlying topological spaces together with a map of sheaves of $\mathbb{F}$-rings on $Y, f^{\#}: \mathcal{O}_{Y} \rightarrow f_{*} \mathcal{O}_{X}$, i.e. for $U \subseteq Y$ open we have $f_{U}^{\#}: \mathcal{O}_{Y}(U) \rightarrow \mathcal{O}_{X}\left(f^{-1} U\right)$ a map of $\mathbb{F}$-rings, such that for

$$
U^{\prime} \subseteq U:\left.f_{U}^{\#}(s)\right|_{f^{-1} U^{\prime}}=f_{U^{\prime}}^{\#}\left(\left.s\right|_{U^{\prime}}\right) .
$$

The $\mathbb{F}$-ringed space $X$ is an $\mathbb{F}$-locally-ringed space if for all $\mathfrak{p} \in X$ the stalk $\mathcal{O}_{X, \mathfrak{p}}$ is a local $\mathbb{F}$-ring, i.e. contains a unique maximal $H$-ideal $\mathfrak{m}_{X, \mathfrak{p}}$. For a map of $\mathbb{F}$-ringed spaces $f: X \rightarrow Y$, and for $\mathfrak{p} \in X$, we get an induced homomorphism of $\mathbb{F}$-rings on the stalks

$$
f_{\mathfrak{p}}^{\#}: \mathcal{O}_{Y, f(\mathfrak{p})}=\underset{f(\mathfrak{p}) \in V}{\lim _{(}} \mathcal{O}_{Y}(V) \rightarrow \underset{\mathfrak{p} \in f^{-1} V}{\lim _{\longrightarrow}} \mathcal{O}_{X}\left(f^{-1} V\right) \rightarrow \underset{\mathfrak{p} \in U}{\lim _{\longrightarrow}} \mathcal{O}_{X}(U)=\mathcal{O}_{X, \mathfrak{p}}
$$

A map $f: X \rightarrow Y$ of $\mathbb{F}$-locally-ringed spaces is a map of $\mathbb{F}$-ringed spaces such that $f_{\mathfrak{p}}^{\#}$ is a local homomorphism for all $\mathfrak{p} \in X$, i.e.

$$
f_{\mathfrak{p}}^{\#}\left(\mathfrak{m}_{Y, f(\mathfrak{p})}\right) \subseteq \mathfrak{m}_{X, \mathfrak{p}} \text { or equivalently }\left(f_{\mathfrak{p}}^{\#}\right)^{-1} \mathfrak{m}_{X, \mathfrak{p}}=\mathfrak{m}_{Y, f(\mathfrak{p})}
$$

We let $\mathbb{F}$-Rings.S $\mathcal{S}$ (respectively $\mathcal{L}$ oc- $\mathbb{F}$ - $\mathcal{R}$ ings- $\mathcal{S} p$ ) denote the category of $\mathbb{F}$-(locally)-ringed spaces.

For a (locally) $\mathbb{F}$-ringed space $\left(X, \mathcal{O}_{X}\right)$ an $\mathcal{O}_{X}$-module $\mathfrak{M}$ is a sheaf of sets over $X$ such that for $U \subseteq X$ open, $\mathfrak{M}(U)$ is an $\mathcal{O}_{X}(U)$-module, these structures being compatible with restrictions, cf. (5.18.2)-(5.18.4). For two $\mathcal{O}_{X}$-modules $\mathfrak{M}, \mathfrak{M}^{\prime}$ a map of sheaves $\varphi: \mathfrak{M} \rightarrow \mathfrak{M}^{\prime}$ is a homomorphism 


\section{J. SHAI HARAN}

of $\mathcal{O}_{X}$-modules if for $U \subseteq X$ open the map $\varphi_{U}: \mathfrak{M}(U) \rightarrow \mathfrak{M}^{\prime}(U)$ is a homomorphism of $\mathcal{O}_{X}(U)$ modules. Thus we have the category $\mathcal{O}_{X}-\mathcal{M o d}$ of $\mathcal{O}_{X}$-modules.

For a homomorphism of $\mathbb{F}$-rings $\varphi: A \rightarrow B$, for $\mathfrak{p} \in \operatorname{Spec}(B)$, we have a unique homomorphism $\varphi_{\mathfrak{p}}: A_{\varphi^{-1} \mathfrak{p}} \rightarrow B_{\mathfrak{p}}$, such that we have a commutative diagram

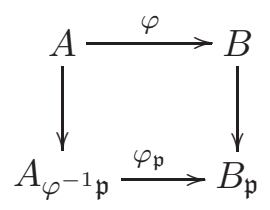

where $\varphi_{\mathfrak{p}}(a / s)=\varphi(a) / \varphi(s)$, and $\varphi_{\mathfrak{p}}$ is a local homomorphism. Thus $A \mapsto \operatorname{Spec}(A)$ is a contravariant

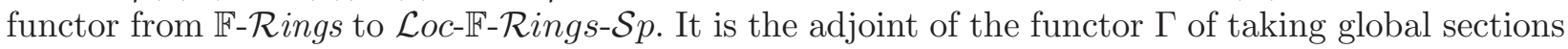

$$
\Gamma\left(X, \mathcal{O}_{X}\right)=\mathcal{O}_{X}(X), \quad \Gamma(f)=f_{Y}^{\#}: \mathcal{O}_{Y}(Y) \rightarrow \mathcal{O}_{X}(X) .
$$

Proposition 6.3. We have

$$
\operatorname{Hom}_{\mathcal{L} o c-\mathbb{F}-\mathcal{R} \text { ings-Spp }}(X, \operatorname{Spec}(A))=\operatorname{Hom}_{\mathbb{F}-\mathcal{R} \text { ings }}\left(A, \mathcal{O}_{X}(X)\right) .
$$

Proof. For an $\mathbb{F}$-locally-ringed space $X$, and for a point $x \in X$, the canonical homomorphism $\phi_{x}$ : $\mathcal{O}_{X}(X) \rightarrow \mathcal{O}_{X, x}$ gives a prime $\mathcal{P}(x)=\phi_{x}^{-1}\left(\mathfrak{m}_{X, x}\right) \in \operatorname{Spec} \mathcal{O}_{X}(X)$. The map $\mathcal{P}: X \rightarrow \operatorname{Spec} \mathcal{O}_{X}(X)$ is continuous:

$$
\mathcal{P}^{-1}(D(f))=\left\{x \in X \mid \phi_{x}(f) \notin \mathfrak{m}_{X, x}\right\}
$$

is open for $f \in \mathcal{O}_{X}(X)$. We have an induced homomorphism

$$
\mathcal{P}_{D(f)}^{\#}: \mathcal{O}_{X}(X)_{f} \rightarrow \mathcal{O}_{X}\left(\left\{x \in X \mid \phi_{x}(f) \notin \mathfrak{m}_{X, x}\right\}\right),
$$

making $\mathcal{P}$ a map of $\mathbb{F}$-ringed spaces, and taking the direct limit over $f$ with $\phi_{x}(f) \notin \mathfrak{m}_{X, x}$ we get

$$
\mathcal{P}_{x}^{\#}: \mathcal{O}_{X}(X)_{\mathcal{P}(x)} \rightarrow \mathcal{O}_{X, x},
$$

showing $\mathcal{P}$ is a map of $\mathbb{F}$-locally-ringed spaces.

To a homomorphism of $\mathbb{F}$-rings $\varphi: A \rightarrow \mathcal{O}_{X}(X)$ we associate the map of $\mathbb{F}$-locally-ringed spaces

$$
X \stackrel{\mathcal{P}}{\longrightarrow} \operatorname{Spec} \mathcal{O}_{X}(X) \stackrel{\operatorname{Spec}(\varphi)}{\longrightarrow} \operatorname{Spec} A .
$$

Conversely, to a map $f: X \rightarrow \operatorname{Spec} A$ of $\mathbb{F}$-locally-ringed spaces (as in Definition 6.1 ) we associate its action on global sections

$$
\Gamma(f)=f_{\text {Spec } A}^{\#}: A=\mathcal{O}_{A}(\operatorname{Spec} A) \rightarrow \mathcal{O}_{X}(X) .
$$

Clearly, $\Gamma(\operatorname{Spec}(\varphi) \circ \mathcal{P})=\varphi$.

Conversely, given a map $f: X \rightarrow \operatorname{Spec} A$ (as in Definition 6.1), for $x \in X$ we have the following commutative diagram:

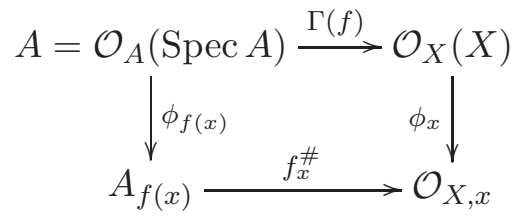

Since $f_{x}^{\#}$ is assumed to be local, $\left(f_{x}^{\#}\right)^{-1}\left(\mathfrak{m}_{X, x}\right)=\mathfrak{m}_{f(x)}$, and by the commutativity of the diagram we get $\Gamma(f)^{-1}(\mathcal{P}(x))=f(x)$, i.e. $f=(\operatorname{Spec} \Gamma(f)) \circ \mathcal{P}$ is the continuous map associated to the 


\section{NON-ADDITIVE GEOMETRY}

homomorphism $\Gamma(f)$. Similarly, for $g \in A$, the commutativity of the diagram

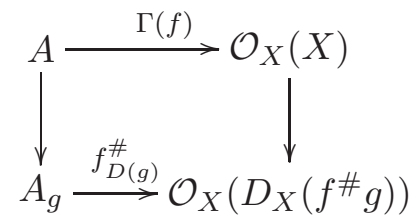

gives $f_{D(g)}^{\#}\left(a / g^{n}\right)=\Gamma(f)(a) /(\Gamma(f)(g))^{n}$, hence $f=(\operatorname{Spec} \Gamma(f)) \circ \mathcal{P}$ as a map of $\mathbb{F}$-locally-ringed spaces.

Corollary 6.3.1. For $\mathbb{F}$-rings $A, B$ :

$$
\operatorname{Hom}_{\mathcal{L} o c-\mathbb{F}-\mathcal{R} \text { ings-Spp }}(\operatorname{Spec} B, \operatorname{Spec} A)=\operatorname{Hom}_{\mathbb{F}-\mathcal{R} i n g s}(A, B) .
$$

\subsection{Zariski $\mathbb{F}$-schemes}

Definition 6.4. A Zariski $\mathbb{F}$-scheme is an $\mathbb{F}$-locally-ringed space $\left(X, \mathcal{O}_{X}\right)$, such that there is a covering by open sets $X=\bigcup_{i} U_{i}$, and the canonical maps

$$
\mathcal{P}:\left(U_{i},\left.\mathcal{O}_{X}\right|_{U_{i}}\right) \rightarrow \operatorname{Spec} \mathcal{O}_{X}\left(U_{i}\right)
$$

are isomorphisms of $\mathbb{F}$-locally-ringed spaces. A morphism of Zariski $\mathbb{F}$-schemes is a map of $\mathbb{F}$-locallyringed spaces. We denote the category of Zariski $\mathbb{F}$-schemes by $\mathcal{Z}$ ar- $\mathbb{F}$-S $\operatorname{sch}$.

Zariski $\mathbb{F}$-schemes can be glued.

Proposition 6.5. Given a set of indices $I$, and for $i \in I$ given $X_{i} \in \mathcal{Z}$ ar $-\mathbb{F}-\mathcal{S} c h$, and for $i \neq j, i$, $j \in I$, an isomorphism $\varphi_{i j}: U_{i j} \stackrel{\sim}{\longrightarrow} U_{j i}$, with $U_{i j} \subseteq X_{i}$ open (and hence $U_{i j}$ are Zariski $\mathbb{F}$-schemes), such that

$$
\begin{gathered}
\varphi_{j i}=\varphi_{i j}^{-1}, \\
\varphi_{i j}\left(U_{i j} \cap U_{i k}\right)=U_{j i} \cap U_{j k} \quad \text { and } \varphi_{j k} \circ \varphi_{i j}=\varphi_{i k} \text { on } U_{i j} \cap U_{i k} .
\end{gathered}
$$

There exists $X \in \mathcal{Z}$ ar- $\mathbb{F}-\mathcal{S}$ ch, and maps $\psi_{i}: X_{i} \rightarrow X$, such that

$\psi_{i}$ is an isomorphism of $X_{i}$ onto the open set $\psi_{i}\left(X_{i}\right) \subseteq X$,

$$
\begin{gathered}
X=\bigcup_{i} \psi_{i}\left(X_{i}\right), \\
\psi_{i}\left(U_{i j}\right)=\psi_{i}\left(X_{i}\right) \cap \psi_{j}\left(X_{j}\right), \\
\psi_{i}=\psi_{j} \circ \varphi_{i j} \text { on } U_{i j} .
\end{gathered}
$$

Proof. The proof is clear: glue the topological spaces and glue the sheaves of $\mathbb{F}$-rings. For $V \subseteq X$ open

$$
\mathcal{O}_{X}(V)=\operatorname{ker}\left\{\prod_{i} \mathcal{O}_{X_{i}}\left(\psi^{-1} V\right) \rightrightarrows \prod_{i, j} \mathcal{O}_{X_{i}}\left(\psi^{-1} V \cap U_{i j}\right)\right\} .
$$

Remark 6.6. Let $f:\left(X, \mathcal{O}_{X}\right) \rightarrow\left(Y, \mathcal{O}_{Y}\right)$ be a map of $\mathbb{F}$-ringed spaces. If $\mathfrak{M}$ is an $\mathcal{O}_{X}$-module, then $f_{*} \mathfrak{M}(V)=\mathfrak{M}\left(f^{-1} V\right), V \subseteq Y$ open, gives rise to an $f_{*} \mathcal{O}_{X}$-module. Using the map $f^{\sharp}: \mathcal{O}_{Y} \rightarrow f_{*} \mathcal{O}_{X}$, we see that $f_{*} \mathfrak{M}$ is naturally an $\mathcal{O}_{Y}$-module.

If $\mathfrak{N}$ is an $\mathcal{O}_{Y}$-module, its inverse image $f^{-1} \mathfrak{N}$ is the sheaf on $X$ associated with the presheaf

$$
U \mapsto \underset{f(\overrightarrow{U) \subseteq} V}{\lim _{\longrightarrow}} \mathfrak{N}(V)
$$

and $f^{-1} \mathfrak{N}$ is an $f^{-1} \mathcal{O}_{Y}$-module. To give the map $f^{\sharp}: \mathcal{O}_{Y} \rightarrow f_{*} \mathcal{O}_{X}$ of sheaves on $Y$ is equivalent to giving the map $f^{\natural}: f^{-1} \mathcal{O}_{Y} \rightarrow \mathcal{O}_{X}$ of sheaves on $X$. Using the map $f^{\natural}$ we can extend scalars, 


\section{J. Shai HARAN}

cf. Proposition 3.23, to form the presheaf

$$
U \mapsto\left(f^{-1} \mathfrak{N}(U)\right)_{\mathcal{O}_{X}(U)}
$$

The sheaf associated to this presheaf is denoted $f^{*} \mathfrak{N}$; it is an $\mathcal{O}_{X}$-module.

The functors of direct image $f_{*}$ and of inverse image $f^{*}$ are adjoint

$$
\operatorname{Hom}_{\mathcal{O}_{X}-\mathcal{M o d}}\left(f^{*} \mathfrak{N}, \mathfrak{M}\right)=\operatorname{Hom}_{\mathcal{O}_{Y}-\mathcal{M o d}}\left(\mathfrak{N}, f_{*} \mathfrak{M}\right) .
$$

For a homomorphism of $\mathbb{F}$-rings $\varphi: A \rightarrow B$, and the associated map $f=\varphi^{*}: \operatorname{Spec} B \rightarrow \operatorname{Spec} A$, and for any $B$-module $M$ with associated $\mathcal{O}_{B}$-module $\widetilde{M}$, cf. Remark 5.18.1, and associated $A$-module $\varphi^{*} M$, cf. (3.22.1-2), we have

$$
\left.f_{*}(\widetilde{M})=\widetilde{\left(\varphi^{*} M\right.}\right)
$$

For an $A$-module $N$ with associated $B$-module $N_{B}$, cf. Proposition 3.23 , we have

$$
f^{*}(\tilde{N})=\widetilde{\left(N_{B}\right)} \text {. }
$$

The theory of quasi-coherent modules over a Zariski $\mathbb{F}$-scheme goes over as in the classical theory, incorporating the notions of $\S 3$. We shall not give the details here, and we give only the basic definitions.

Theorem 6.7. Let $A$ be an $\mathbb{F}$-ring. Let $U \subseteq X=\operatorname{Spec} A$ be an open compact subset, and $\mathfrak{M}$ an $\left.\mathcal{O}_{X}\right|_{U}$-module. The following conditions are equivalent:

(1) there exists an A-module $M$ and an isomorphism $\left.\widetilde{M}\right|_{U} \cong \mathfrak{M}$;

(2) there exists an open affine cover $U=\bigcup_{i \in I} U_{i}, U_{i}=\operatorname{Spec} A_{i}$, and for $i \in I$ there are $A_{i}$-modules $M_{i}$ such that $\left.\widetilde{M}_{i} \cong \mathfrak{M}\right|_{U_{i}}$

(3) for every open affine $\operatorname{Spec} B \subseteq U$ there exists a $B$-module $M$ such that $\left.\widetilde{M} \cong \mathfrak{M}\right|_{\operatorname{Spec} B}$;

(4) for every $f \in A_{[1],[1]}$ the restriction induces an isomorphism of $A_{f}$-modules

$$
\Gamma(U, \mathfrak{M})_{f} \stackrel{\sim}{\longrightarrow} \Gamma\left(D_{A}(f), \mathfrak{M}\right) .
$$

Proof. See [Gro60, 1.4].

If $\mathfrak{M}$ satisfies the conditions of Theorem 6.7 we say it is quasi-coherent. Similarly for a Zariski $\mathbb{F}$-scheme $X$, replacing in (2) or in (3) $U$ by $X$ we get the notion of quasi-coherent $\mathcal{O}_{X}$-module. We denote by $\mathcal{Q C}-\mathcal{O}_{X}-\mathcal{M}$ od the category of quasi-coherent $\mathcal{O}_{X}$-modules. For an affine scheme $X=$ $\operatorname{Spec} A$, the functors $M \mapsto \widetilde{M}, \mathfrak{M} \mapsto \Gamma(X, \mathfrak{M})$, give inverse isomorphisms of categories

$$
A-\mathcal{M o d} \stackrel{\sim}{\longrightarrow} \mathcal{Q}-\mathcal{O}_{A}-\mathcal{M} o d
$$

\section{3 $\mathbb{F}$-schemes and the compactified $\overline{\operatorname{Spec} \mathbb{Z}}$}

The category of $\mathbb{F}$-locally-ringed spaces has inverse limits. Given an inverse system $\left\{X_{j}, \mathcal{O}_{X_{j}}\right\}_{j \in J}$, where $J$ is a partially ordered set, and for $j_{1} \geqslant j_{2}$ in $J$ we are given maps $\pi_{j_{2}}^{j_{1}}: X_{j_{1}} \rightarrow X_{j_{2}}$ such that $\pi_{j}^{j}=\operatorname{id}_{X_{j}}$, and $\pi_{j_{3}}^{j_{2}} \circ \pi_{j_{2}}^{j_{1}}=\pi_{j_{3}}^{j_{1}}$ for $j_{1} \geqslant j_{2} \geqslant j_{3}$, and where we always assume that $J$ is directed (for $j_{1}, j_{2} \in J$ there exists $j \in J$ with $j \geqslant j_{1}, j \geqslant j_{2}$ ), the inverse limit $\lim _{J} X_{j}$ is constructed as follows. As a topological space it is the inverse limit topological space, i.e. $\lim _{J} X_{j}$ is the inverse limit of the $X_{j}$ as a set, together with the topology having as a basis for open sets the sets of the form $\pi_{j}^{-1}(U)$, where $\pi_{j}: \lim _{J} X_{j} \rightarrow X_{j}$ are the projections, and where $U \subseteq X_{j}$ are arbitrary open sets in $X_{j}$ (we can take the $U$ to vary over a basis for the topology of the $X_{j}$ ). Then on the topological space $X=\lim _{J} X_{j}$ we have the directed system of sheaves of $\mathbb{F}$-rings $\left\{\pi_{j}^{-1} \mathcal{O}_{j}\right\}$ and the direct limit, i.e. the sheaf associated with the pre-sheaf $U \mapsto \lim _{J} \pi_{j}^{-1} \mathcal{O}_{j}(U)$ is the sheaf $\mathcal{O}_{X}$ on $X=\lim _{J} X_{j}$ 


\section{NON-ADDITIVE GEOMETRY}

satisfying the universal property of the inverse limit in the category $\mathcal{L}$ oc- $\mathbb{F}$ - $\mathcal{R}$ ings- $\mathcal{S}$. For a point $x=\left\{x_{j}\right\} \in X$, the stalk $\mathcal{O}_{X, x}$ is the direct limit of the stalks $\mathcal{O}_{X_{j}, x_{j}}$, and hence is indeed a local $\mathbb{F}$-ring, and $\left(\pi_{j}^{\sharp}\right)_{x}: \mathcal{O}_{X_{j}, x_{j}} \rightarrow \mathcal{O}_{X, x}$ is a local homomorphism. For an open set $U \subseteq X$ the sections $\mathcal{O}_{X}(U)$ can alternatively be described as the maps

$$
s: U \rightarrow \coprod_{x \in U} \mathcal{O}_{X, x}, \quad s(x) \in \mathcal{O}_{X, x},
$$

such that for any $x=\left\{x_{j}\right\} \in U$ there exists an open set $U_{j} \subseteq X_{j}$ for some $j \in J$, such that $x_{j} \in U_{j}, \pi^{-1}\left(U_{j}\right) \subseteq U$, and there is a section $s_{j} \in \mathcal{O}_{X_{j}}\left(U_{j}\right)$, such that for all $y \in \pi^{-1}\left(U_{j}\right)$, we have $s(y)=\left(\pi_{j}^{\sharp}\right)_{y}\left(\left.s_{j}\right|_{\pi_{j}(y)}\right)$. In the case that the $X_{j}$ are all affine Zariski $\mathbb{F}$-schemes, $X_{j}=\operatorname{Spec}\left(A_{j}\right)$, the inverse limit $X=\lim _{J} X_{j}$ is again an affine Zariski $\mathbb{F}$-scheme, namely $X=\operatorname{Spec}(A)$, where $A=\varliminf_{J} A_{j}$ is the direct limit of the $\mathbb{F}$-rings $A_{j}$. But in the case that the $X_{j}$ are Zariski $\mathbb{F}$-schemes, the inverse limit $X=\lim _{J} X_{j}$ need not be a Zariski $\mathbb{F}$-scheme, and the category $\mathcal{Z}$ ar- $\mathbb{F}$-S $\mathcal{S}$ ch does not have inverse limits $\left(X=\lim _{J} X_{j}\right.$ will be a Zariski $\mathbb{F}$-scheme if the maps $\pi_{j_{2}}^{j_{1}}: X_{j_{1}} \rightarrow X_{j_{2}}$ are affine).

Definition 6.8. The category of $\mathbb{F}$-schemes, $\mathbb{F}$ - $\mathcal{S} c h$, is the category of pro-objects of the category of Zariski $\mathbb{F}$-schemes.

Thus the objects of $\mathbb{F}-\mathcal{S} c h$ are inverse systems $X=\left\{X_{j}\right\}_{j \in J}$, where the $X_{j}$ are Zariski $\mathbb{F}$-schemes, and where $J$ is an arbitrary directed set, and the maps in $\mathbb{F}$-S $\mathcal{S}$ ch from such an object to another object $Y=\left\{Y_{i}\right\}_{i \in I}$ are given by

$$
\operatorname{Hom}_{\mathbb{F}-\mathcal{S} c h}(X, Y)=\lim _{I}\left(\underset{J}{\lim _{J}} \operatorname{Hom}_{\mathcal{Z} a r-\mathbb{F}-\mathcal{S} c h}\left(X_{j}, Y_{i}\right)\right),
$$

i.e. the maps $\varphi: X \rightarrow Y$ are given by a collection of maps $\varphi_{i}^{j}: X_{j} \rightarrow Y_{i}$ defined for all $i \in I$ and for $j \geqslant \sigma(i)$ sufficiently large (depending on $i$ ), and are inductive in the index $j$ and projective in the index $i$ : for all $i \in I$, and for $j_{1} \geqslant j_{2}$ sufficiently large in $J$,

$$
\varphi_{i}^{j_{1}}=\varphi_{i}^{j_{2}} \circ \pi_{j_{2}}^{j_{1}} \quad\left(\text { here } \pi_{j_{2}}^{j_{1}}: X_{j_{1}} \rightarrow X_{j_{2}}\right) ;
$$

and for all $i_{1} \geqslant i_{2}$ in $I$, and for $j$ sufficiently large in $J$,

$$
\pi_{i_{2}}^{i_{1}} \circ \varphi_{i_{1}}^{j}=\varphi_{i_{2}}^{j} \quad\left(\text { here } \pi_{i_{2}}^{i_{1}}: Y_{i_{1}} \rightarrow Y_{i_{2}}\right) .
$$

The maps $\left\{\varphi_{i}^{j}: X \rightarrow Y\right\}$ and $\left\{\widetilde{\varphi}_{i}^{j}: X \rightarrow Y\right\}$ are considered equivalent if for all $i \in I$, and for $j$ sufficiently large in $J, \varphi_{i}^{j}=\widetilde{\varphi}_{i}^{j}$. The identity map of $\left\{X_{j}\right\}$ is represented by $\left\{\pi_{j_{2}}^{j_{1}}: X_{j_{1}} \rightarrow X_{j_{2}}\right\}_{j_{1}} \geqslant j_{2}$. The composition of $\varphi=\left\{\varphi_{i}^{j}\right\}_{j \geqslant \sigma(i)}:\left\{X_{j}\right\}_{J} \rightarrow\left\{Y_{i}\right\}_{I}$ with $\widetilde{\varphi}=\left\{\widetilde{\varphi}_{k}^{i}\right\}_{i \geqslant \widetilde{\sigma}(k)}:\left\{Y_{i}\right\}_{I} \rightarrow\left\{Z_{k}\right\}_{K}$ is given by

$$
\widetilde{\varphi} \circ \varphi=\left\{\widetilde{\varphi}_{k}^{i} \circ \varphi_{i}^{j}\right\}_{j \geqslant \sigma(\widetilde{\sigma}(k))} .
$$

Note that there is always a map (with Hom in $\mathcal{L}$ oc- $\mathbb{F}-\mathcal{R}$ ings- $\mathcal{S} p$ )

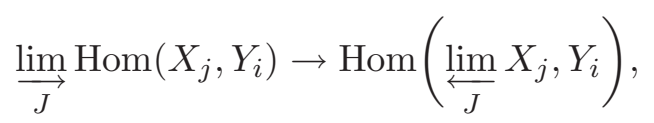

and by definition

$$
\varliminf_{I}^{\lim } \operatorname{Hom}\left(\varliminf_{J}^{\lim } X_{j}, Y_{i}\right) \stackrel{\sim}{\longrightarrow} \operatorname{Hom}\left(\varliminf_{J}^{\lim } X_{j}, \varliminf_{I}^{\lim } Y_{i}\right) .
$$

Composing (6.8.5) and (6.8.6) we obtain

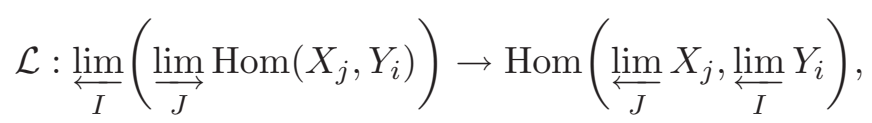




\section{J. Shai HARAN}

i.e. a functor

$$
\mathcal{L}: \mathbb{F}-\mathcal{S} c h \rightarrow \mathcal{L} \text { oc- } \mathbb{F}-\mathcal{R} \text { ings-Sp }, \quad \mathcal{L}\left(\left\{X_{j}\right\}_{J}\right)=\underbrace{\lim }_{J} X_{j}
$$

Example 6.9. The compactified $\overline{\text { Spec } \mathbb{Z}}$. Fix a square-free integer $N \geqslant 2$. Let $A_{N}=\mathbb{F}(\mathbb{Z}[1 / N]) \cap \mathcal{O}_{\mathbb{Q}, \eta}$; it is the $\mathbb{F}$-ring with

$$
\left(A_{N}\right)_{Y, X}=\left\{a \in \mathbb{F}\left(\mathbb{Z}\left[\frac{1}{N}\right]\right)_{Y, X}:|a|_{\eta} \leqslant 1\right\},
$$

the $Y \times X$ matrices with values in $\mathbb{Z}[1 / N]$ and with (real) operator norm bounded by 1 . The map $j: A_{N} \rightarrow \mathbb{F}(\mathbb{Z}[1 / N])$ defines the basic open set

$$
j^{*}: \operatorname{Spec} \mathbb{Z}\left[\frac{1}{N}\right] \cong \operatorname{Spec} \mathbb{F}\left(\mathbb{Z}\left[\frac{1}{N}\right]\right) \cong D_{A_{N}}\left(\frac{1}{N}\right) \hookrightarrow \operatorname{Spec} A_{N}
$$

Indeed, it is easy to check that the map $\left(A_{N}\right)_{1 / N} \rightarrow \mathbb{F}(\mathbb{Z}[1 / N])$ is an isomorphism of $\mathbb{F}$-rings, where $\left(A_{N}\right)_{1 / N}$ is the localization of $A_{N}$ with respect to $1 / N \in\left(A_{N}\right)_{[1],[1]}$ : every matrix $a \in$ $\mathbb{F}(\mathbb{Z}[1 / N])_{Y, X}$ can be written as $a=\left(a / N^{k}\right) /\left(1 / N^{k}\right)$, and for $k$ sufficiently large $a / N^{k} \in\left(A_{N}\right)_{Y, X}$. The space Spec $A_{N}$ contains also the closed point

$$
\eta=i^{*}\left(\mathfrak{m}_{\mathbb{Q}, \eta}\right)=\left\{\left.a \in\left(A_{N}\right)_{[1],[1]}|| a\right|_{\eta}<1\right\}=\mathbb{Z}\left[\frac{1}{N}\right] \cap(-1,1),
$$

and it is the 'real prime' given by $i: A_{N} \hookrightarrow \mathcal{O}_{\mathbb{Q}, \eta}$. But note that $\eta$ is 'very close': the only open set containing $\eta$ is the hole space, since for any non-trivial basic open set $D_{A_{N}}(f)$, say $f=p / N^{k}$, $p$ prime not dividing $N$ and $p<N^{k}$, we have

$$
\left(A_{N}\right)_{f}=\mathbb{F}\left(\mathbb{Z}\left[\frac{1}{N \cdot p}\right]\right)
$$

(indeed, every matrix $a \in \mathbb{F}(\mathbb{Z}[1 / N \cdot p])_{Y, X}$ can be written as $a=\left(p / N^{k}\right)^{l} \cdot a / f^{l}$, and for $l$ sufficiently large $\left.\left(p / N^{k}\right)^{l} \cdot a \in\left(A_{N}\right)_{Y, X}\right)$, and so

$$
D_{A_{N}}(f)=\operatorname{Spec}\left(A_{N}\right)_{f}=\operatorname{Spec} \mathbb{F}\left(\mathbb{Z}\left[\frac{1}{N \cdot p}\right]\right) \cong \operatorname{Spec} \mathbb{Z}\left[\frac{1}{N \cdot p}\right]
$$

does not contain $\eta$. Further, $\eta$ contains all the primes of $A_{N}$, it is the (unique) maximal $H$-ideal of $A_{N}$, and $A_{N}$ is a local $\mathbb{F}$-ring (of 'Krull' dimension 2).

Let $X_{N}$ be the Zariski $\mathbb{F}$-scheme obtained by gluing Spec $A_{N}$ with $\operatorname{Spec} \mathbb{F}(\mathbb{Z})$ along the common open set $\operatorname{Spec} \mathbb{F}(\mathbb{Z}[1 / N])$ cf. Proposition 6.5 or [Hart77, p. 75, Example 2.3.5]. The open sets of $X_{N}$ are the open sets of Spec $\mathbb{Z}$, and sets of the form $U \cup\{\eta\}$ with $\operatorname{Spec} \mathbb{Z}[1 / N] \subseteq U \subseteq \operatorname{Spec} \mathbb{Z}$. For an open set $U=\operatorname{Spec} \mathbb{Z}[1 / M] \subseteq \operatorname{Spec} \mathbb{Z}$, we have $\mathcal{O}_{X_{N}}(U)=\mathbb{F}(\mathbb{Z}[1 / M])$, and for such a set $U=\operatorname{Spec} \mathbb{Z}[1 / M]$ with $M$ dividing $N$, we have $\mathcal{O}_{X_{N}}(U \cup\{\eta\})=A_{M}$.

For $N$ dividing $M$ we have commutative diagrams
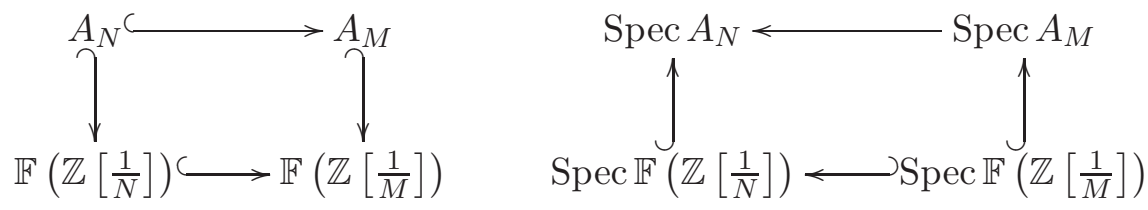

and we obtain a map $\pi_{N}^{M}: X_{M} \rightarrow X_{N}$. Note that $\pi_{N}^{M}$ is a bijection on points, and further $\left(\pi_{N}^{M}\right)_{*} \mathcal{O}_{X_{M}}=\mathcal{O}_{X_{N}}$, i.e. $\left(\pi_{N}^{M}\right)^{\sharp}$ is the identity, but there are more open sets in $X_{M}$ than there are in $X_{N}$. We need all these open sets, for all $N$, and so we pass to the inverse limit. The 'compactified Spec $\mathbb{Z}$ ' is the $\mathbb{F}$-scheme given by the inverse system $\left\{X_{N}\right\}$, we denote it by $\overline{\text { Spec } \mathbb{Z}}$. The set of indices is the set of square-free integers $N \geqslant 2$, and the order relation is that of divisibility. 
Note that the $\mathbb{F}$-locally-ringed space $\mathcal{L}(\overline{\operatorname{Spec} \mathbb{Z}})=\lim _{N} X_{N}$ has for points $\operatorname{Spec} \mathbb{Z} \cup\{\eta\}$, with open sets of the form $U$ or $U \cup\{\eta\}$ with $U$ an arbitrary open set of $\operatorname{Spec} \mathbb{Z}$ (hence $\overline{\operatorname{Spec} \mathbb{Z}}$ is of 'Krull' dimension 1). Note that each $X_{N}$ is compact, cf. Proposition 4.12, and hence $\mathcal{L}(\overline{\mathrm{Spec} \mathbb{Z}})$ is compact. Furthermore, the local $\mathbb{F}$-ring $\mathcal{O}_{\overline{\mathrm{Spec}} \mathbb{Z}, \eta}$ is just $\mathcal{O}_{\mathbb{Q}, \eta}$ (while the local $\mathbb{F}$-ring $\mathcal{O}_{X_{N}, \eta}$ is only $A_{N}$ ). For an open set $U=\operatorname{Spec} \mathbb{Z}[1 / N]$ we have

$$
\mathcal{O}_{\overline{\operatorname{Spec} \mathbb{Z}}}(U)=\mathbb{F}\left(\mathbb{Z}\left[\frac{1}{N}\right]\right)
$$

and

$$
\mathcal{O}_{\overline{\mathrm{Spec} \mathbb{Z}}}(U \cup\{\eta\})=A_{N} .
$$

The global sections $\mathcal{O}_{\overline{\text { Spec } \mathbb{Z}}}(\overline{\text { Spec } \mathbb{Z}})$ are the $\mathbb{F}$-ring $\mathbb{F}\langle\{ \pm 1\} \cup\{0\}\rangle$.

Example 6.10. Similarly for a number field $K$, with ring of integers $\mathcal{O}_{K}$, and with real primes $\left\{\eta_{i}\right\}, i=1, \ldots, r\left(=r_{\mathbb{R}}+r_{\mathbb{C}}\right)$, let $A_{N, i}=\mathbb{F}\left(\mathcal{O}_{K}[1 / N]\right) \cap \mathcal{O}_{K, \eta_{i}}$ be the $\mathbb{F}$-ring with

$$
\left(A_{N, i}\right)_{Y, X}=\left\{a \in \mathbb{F}\left(\mathcal{O}_{K}\left[\frac{1}{N}\right]\right):|a|_{\eta_{i}} \leqslant 1\right\},
$$

the $Y \times X$ matrices with values in $\mathcal{O}_{K}[1 / N]$ and with $\eta_{i}$-operator norm bounded by 1 . Let $X_{N}$ be the Zariski $\mathbb{F}$-scheme obtained by gluing $\left\{\operatorname{Spec} A_{N, i}\right\}_{i=1, \ldots, r}$ and $\left\{\operatorname{Spec} \mathbb{F}\left(\mathcal{O}_{K}\right)\right\}$ along the common open set $\operatorname{Spec} \mathbb{F}\left(\mathcal{O}_{K}[1 / N]\right)$. For $N$ dividing $M$ we obtain a map $\pi_{N}^{M}: X_{M} \rightarrow X_{N}$, with $\left.\pi_{N}^{M}\right|_{\operatorname{Spec} A_{M, i}}$ induced by $A_{N, i} \subseteq A_{M, i}$. The inverse system $\left\{X_{N}\right\}$ is the $\mathbb{F}$-scheme $\overline{\operatorname{Spec} \mathcal{O}_{K}}$, the compactification of $\operatorname{Spec} \mathcal{O}_{K}$. The space $\mathcal{L}\left(\overline{\operatorname{Spec} \mathcal{O}_{K}}\right)=\lim _{N} X_{N}$ has for points $\operatorname{Spec} \mathcal{O}_{K} \cup\left\{\eta_{i}\right\}_{i \leqslant r}$, and open sets are of the form $U \cup\left\{\eta_{i}\right\}_{i \in I}$ with $U$ open in $\operatorname{Spec} \mathcal{O}_{K}$, and $I \subseteq\{1, \ldots, r\}$ a subset (and hence it is of 'Krull' dimension 1). The local $\mathbb{F}$-ring $\mathcal{O}_{\overline{\operatorname{Spec} \mathcal{O}_{K}}}, \eta_{i}$ is the ring $\mathcal{O}_{K, \eta_{i}}$. The global sections $\mathcal{O}_{\overline{\operatorname{Spec} \mathcal{O}_{K}}}\left(\overline{\operatorname{Spec} \mathcal{O}_{K}}\right)$ are the $\mathbb{F}$-ring $\mathbb{F}\left\langle\mu_{K} \cup\{0\}\right\rangle, \mu_{K}$ the group of roots of unity in $\mathcal{O}_{K}^{*}$.

\section{Fibred products}

We show that the category of $\mathbb{F}$-rings has fibred sums, and we deduce that the category of (Zariski) F-schemes has fibred products.

\subsection{Fibred sums of $\mathbb{F}$-rings}

Theorem 7.1. The category $\mathbb{F}$-R ings has fibred sums: given homomorphism of $\mathbb{F}$-rings $\varphi^{0}: A \rightarrow B^{0}$, $\varphi^{1}: A \rightarrow B^{1}$, there exists an $\mathbb{F}$-ring $B^{0} \odot_{A} B^{1}$, and homomorphisms $\psi^{i}: B^{i} \rightarrow B^{0} \odot_{A} B^{1}, i=0,1$, such that $\psi^{0} \circ \varphi^{0}=\psi^{1} \circ \varphi^{1}$ and for any $\mathbb{F}$-ring $C$ one has

$$
\begin{gathered}
\operatorname{Hom}_{\mathbb{F}-\mathcal{R} \text { ing }}\left(B^{0} \odot_{A} B^{1}, C\right)=\operatorname{Hom}_{\mathbb{F}-\mathcal{R} \text { ing }}\left(B^{0}, C\right) \prod_{\operatorname{Hom}_{\mathbb{F}-\mathcal{R} i n g}(A, C)} \operatorname{Hom}_{\mathbb{F}-\mathcal{R} \text { ing }}\left(B^{1}, C\right), \\
f \mapsto\left(f \circ \psi^{0}, f \circ \psi^{1}\right) .
\end{gathered}
$$

So given homomorphisms $f^{0}: B^{0} \rightarrow C, f^{1}: B^{1} \rightarrow C$, such that $f^{0} \circ \varphi^{0}=f^{1} \circ \varphi^{1}$, there exists a unique homomorphism $f^{0} \odot f^{1}: B^{0} \odot_{A} B^{1} \rightarrow C$, such that $\left(f^{0} \odot f^{1}\right) \circ \psi^{i}=f^{i}$.

Proof. For $X, Y \in|\mathbb{F}|$, denote by $\mathcal{B}_{Y, X}$ the set of all sequences $\left(b_{l}, b_{l-1}, \ldots, b_{\delta}\right)$, where $\delta=0,1$, $l \geqslant \delta, b_{2 j} \in B^{0}, b_{2 j+1} \in B^{1}$, the range of $b_{j}$ is the domain of $b_{j+1}$, the range of $b_{l}$ is $Y$, the domain of $b_{\delta}$ is $X$. On $\mathcal{B}_{Y, X}$ let $\sim$ denote the equivalence relation generated by the following relations (7.1.1), (7.1.2) and (7.1.3):

$$
\left(\ldots, b_{j+1} \circ \varphi^{j+1}(a), b_{j}, \ldots\right) \sim\left(\ldots, b_{j+1}, \varphi^{j}(a) \circ b_{j}, \ldots\right), \quad a \in A,
$$




\section{J. Shai HARAN}

where we write $\varphi^{j}$ for $\varphi^{j(\bmod 2)}$;

$$
\left(\ldots, b_{j+1}, f, b_{j-1}, \ldots\right) \sim\left(\ldots, b_{j+1} \circ f \circ b_{j-1}, \ldots\right), \quad f \in \mathbb{F},
$$

and these relations include also the boundary cases

$$
\left(f, b_{l}, \ldots\right) \sim\left(f \circ b_{l}, \ldots\right), \quad\left(\ldots, b_{\delta}, f\right) \sim\left(\ldots, b_{\delta} \circ f\right), \quad f \in \mathbb{F} ;
$$

and

$$
\begin{aligned}
& \left(\ldots, b_{j+1} \circ\left(\left(b \otimes \operatorname{id}_{\bar{Y}}\right) \oplus \operatorname{id}_{\bar{Y}}\right),\left(\operatorname{id}_{X} \otimes \bar{b}_{j}\right) \oplus \overline{\bar{b}}_{j}, b_{j-1}, \ldots\right) \\
& \quad \sim\left(\ldots, b_{j+1},\left(\operatorname{id}_{Y} \otimes \bar{b}_{j}\right) \oplus \overline{\bar{b}}_{j},\left(\left(b \otimes \mathrm{id}_{\bar{X}}\right) \oplus \mathrm{id}_{\bar{X}}\right) \circ b_{j-1}, \ldots\right), \quad \text { for } b \in B_{Y, X}^{j+1}, \bar{b}_{j} \in B_{\bar{Y}, \bar{X}}^{j}, \overline{\bar{b}}_{j} \in B_{\overline{\bar{Y}}, \overline{\bar{X}}}^{j} .
\end{aligned}
$$

Write $B_{Y, X}=\mathcal{B}_{Y, X} / \sim$ for the collection of equivalence classes,

$$
\mathbf{B}=\coprod_{Y, X \in|\mathbb{F}|} B_{Y, X}
$$

We are going to prove that $\mathbf{B}$ will give the required $B^{0} \odot_{A} B^{1}$. We define composition $\circ$ on $\mathbf{B}$

$$
B_{Z, Y} \times B_{Y, X} \rightarrow B_{Z, X}
$$

by

$$
\left(b_{l^{\prime}}^{\prime}, \ldots, b_{\delta^{\prime}}^{\prime}\right) / \sim \circ\left(b_{l}, \ldots, b_{\delta}\right) / \sim \stackrel{\text { def }}{=} \begin{cases}\left(b_{l^{\prime}}^{\prime}, \ldots, b_{\delta^{\prime}}^{\prime}, b_{l}, \ldots, b_{\delta}\right) / \sim, & \delta^{\prime} \not \equiv l(\bmod 2), \\ \left(b_{l^{\prime}}^{\prime}, \ldots, b_{\delta^{\prime}}^{\prime} \circ b_{l}, \ldots, b_{\delta}\right) / \sim, & \delta^{\prime} \equiv l(\bmod 2) .\end{cases}
$$

This is well defined, independent of the chosen representatives: we have to show that changing representatives to equivalent ones on the left of (7.1.4) will give an equivalent result on the right of (7.1.4). Since elements of $\mathcal{B}_{Y, X}$ are equivalent if and only if they are connected by a 'path' made up of the 'moves' (7.1.1) or (7.1.2) or (7.1.3), it is enough to check that changing representatives by one of the three moves (7.1.1) or (7.1.2) or (7.1.3) gives equivalent results. This follows by associativity of $\circ$. It then follows that $\circ$ is associative, has identities, and $\mathbf{B}$ is a category. We have functors $\psi^{i}: B^{i} \rightarrow \mathbf{B}, \psi^{i}\left(b_{i}\right)=\left(b_{i}\right) / \sim$ for $b_{i} \in B^{i}$, and $\psi^{0} \circ \varphi^{0}=\psi^{1} \circ \varphi^{1}$ since by (7.1.1), (7.1.2)

$$
\left(\varphi^{0}(a)\right) \sim\left(\varphi^{0}(a), \mathrm{id}\right) \sim\left(\mathrm{id}, \varphi^{1}(a)\right) \sim\left(\varphi^{1}(a)\right) .
$$

Since the zero map $0_{Y, X}$ is in $\mathbb{F}$, and $0_{Y, X}$ composed with anything gives again a zero map, we see that $[0]$ is the initial and final object of $\mathbf{B}$.

We next define the direct sum of two elements $\left(b_{l^{\prime}}^{\prime}, \ldots, b_{\delta^{\prime}}^{\prime}\right) / \sim,\left(b_{l}, \ldots, b_{\delta}\right) / \sim$ of $\mathbf{B}$. First note that by adding identities we may assume that $l^{\prime}=l, \delta^{\prime}=\delta$. We can then define:

$$
\left(b_{l}^{\prime}, \ldots, b_{\delta}^{\prime}\right) / \sim \oplus\left(b_{l}, \ldots, b_{\delta}\right) / \sim \stackrel{\text { def }}{=}\left(b_{l}^{\prime} \oplus b_{l}, \ldots, b_{\delta}^{\prime} \oplus b_{\delta}\right) / \sim .
$$

We claim this is well defined, independent of the chosen representatives, and again it is enough to show that if we change the representative $\left(b_{l}, \ldots, b_{\delta}\right)$ by one of the three moves (7.1.1), (7.1.2), (7.1.3), we get equivalent results.

For the move (7.1.1): since $\varphi^{j+1}(\mathrm{id})=\mathrm{id}$ we have

$$
\begin{aligned}
& \left(\ldots, b_{j+1}^{\prime} \oplus\left(b_{j+1} \circ \varphi^{j+1}(a)\right), b_{j}^{\prime} \oplus b_{j}, \ldots\right) \\
& \quad=\left(\ldots,\left(b_{j+1}^{\prime} \oplus b_{j+1}\right) \circ \varphi^{j+1}(\mathrm{id} \oplus a), b_{j}^{\prime} \oplus b_{j}, \ldots\right) \\
& \quad \stackrel{(7.1 .1)}{\sim}\left(\ldots, b_{j+1}^{\prime} \oplus b_{j+1}, \varphi^{j}(\mathrm{id} \oplus a) \circ\left(b_{j}^{\prime} \oplus b_{j}\right), \ldots\right) \\
& \quad=\left(\ldots, b_{j+1}^{\prime} \oplus b_{j+1}, b_{j}^{\prime} \oplus\left(\varphi^{j}(a) \circ b_{j}\right), \ldots\right) .
\end{aligned}
$$




\section{NON-ADDITIVE GEOMETRY}

For the move (7.1.2):

$$
\begin{aligned}
\left(b_{l}^{\prime}\right. & \left.\oplus \mathrm{id}, \ldots, b_{j}^{\prime} \oplus b_{j+2}, b_{j-1}^{\prime} \oplus\left(b_{j+1} \circ f \circ b_{j-1}\right), \ldots\right) \\
& =\left(\ldots, b_{j}^{\prime} \oplus b_{j+2},\left(\mathrm{id} \oplus b_{j+1}\right) \circ(\mathrm{id} \oplus f) \circ\left(b_{j-1}^{\prime} \oplus b_{j-1}\right), \ldots\right) \\
& \stackrel{(7.1 .2)}{\sim}\left(b_{l}^{\prime} \oplus \mathrm{id}, \ldots, b_{j}^{\prime} \oplus b_{j+2}, \mathrm{id} \oplus b_{j+1}, \mathrm{id} \oplus f, b_{j-1}^{\prime} \oplus b_{j-1}, \ldots\right) \\
& =\left(b_{l}^{\prime} \oplus \mathrm{id}, \ldots,\left(\mathrm{id} \oplus b_{j+3}\right) \circ\left(b_{j+1}^{\prime} \oplus \mathrm{id}\right),\left(\mathrm{id} \oplus b_{j+2}\right) \circ\left(b_{j}^{\prime} \oplus \mathrm{id}\right), \mathrm{id} \oplus b_{j+1}, \mathrm{id} \oplus f, b_{j-1}^{\prime} \oplus b_{j-1}, \ldots\right) \\
& \stackrel{(7.1 .3)}{\sim}\left(b_{l}^{\prime} \oplus \mathrm{id}, \ldots,\left(\mathrm{id} \oplus b_{j+3}\right) \circ\left(b_{j+1}^{\prime} \oplus \mathrm{id}\right), \mathrm{id} \oplus b_{j+2}, \mathrm{id} \oplus b_{j+1}, b_{j}^{\prime} \oplus f, b_{j-1}^{\prime} \oplus b_{j-1}, \ldots\right) \\
& \stackrel{(7.1 .3)}{\sim}\left(b_{l}^{\prime} \oplus \mathrm{id}, \ldots, \mathrm{id} \oplus b_{j+3}, \mathrm{id} \oplus b_{j+2}, b_{j+1}^{\prime} \oplus b_{j+1}, b_{j}^{\prime} \oplus f, b_{j-1}^{\prime} \oplus b_{j-1}, \ldots\right) \stackrel{(7.1 .3)}{\sim} \\
& \quad \ldots \stackrel{(7.1 .3)}{\sim}\left(b_{l}^{\prime} \oplus b_{l}, \ldots, b_{j+1}^{\prime} \oplus b_{j+1}, b_{j}^{\prime} \oplus f, b_{j-1}^{\prime} \oplus b_{j-1}, \ldots\right) .
\end{aligned}
$$

For the move (7.1.3):

$$
\begin{aligned}
& \left(\ldots, b_{j+1}^{\prime} \oplus\left(b_{j+1} \circ((b \otimes \mathrm{id}) \oplus \mathrm{id})\right), b_{j}^{\prime} \oplus\left(\left(\mathrm{id} \otimes \bar{b}_{j}\right) \oplus \overline{\bar{b}}_{j}\right), \ldots\right) \\
& \quad=\left(\ldots,\left(b_{j+1}^{\prime} \oplus b_{j+1}\right) \circ(\mathrm{id} \oplus(b \otimes \mathrm{id}) \oplus \mathrm{id}), b_{j}^{\prime} \oplus\left(\mathrm{id} \otimes \bar{b}_{j}\right) \oplus \overline{\bar{b}}_{j}, b_{j-1}^{\prime} \oplus b_{j-1}, \ldots\right) \\
& \quad \stackrel{(7.1 .3)}{\sim}\left(\ldots, b_{j+1}^{\prime} \oplus b_{j+1}, b_{j}^{\prime} \oplus\left(\mathrm{id} \otimes \bar{b}_{j}\right) \oplus \overline{\bar{b}}_{j},(\mathrm{id} \oplus(b \otimes \mathrm{id}) \oplus \mathrm{id}) \circ\left(b_{j-1}^{\prime} \oplus b_{j-1}\right), \ldots\right) \\
& \quad=\left(\ldots, b_{j+1}^{\prime} \oplus b_{j+1}, b_{j}^{\prime} \oplus\left(\left(\mathrm{id} \otimes \bar{b}_{j}\right) \oplus \overline{\bar{b}}_{j}\right), b_{j-1}^{\prime} \oplus((b \otimes \mathrm{id}) \oplus \mathrm{id}) \circ b_{j-1}, \ldots\right) .
\end{aligned}
$$

Similarly, to define the tensor product of $\left(b_{l^{\prime}}^{\prime}, \ldots, b_{\delta^{\prime}}^{\prime}\right) / \sim$ and $\left(b_{l}, \ldots, b_{\delta}\right) / \sim$, we may assume $l=l^{\prime}, \delta=\delta^{\prime}$, and we let

$$
\left(b_{l}^{\prime}, \ldots, b_{\delta}^{\prime}\right) / \sim \otimes\left(b_{l}, \ldots, b_{\delta}\right) / \sim \stackrel{\text { def }}{=}\left(b_{l}^{\prime} \otimes b_{l}, \ldots, b_{\delta}^{\prime} \otimes b_{\delta}\right) / \sim .
$$

Again this is well defined: changing the representatives $\left(b_{l}, \ldots, b_{\delta}\right)$ by one of the moves (7.1.1), (7.1.2), (7.1.3) does not change the result. The proofs for (7.1.1) and (7.1.2) are exactly as for the direct sum with $\oplus$ replaced everywhere by $\otimes$.

The proof for move (7.1.3) is similar:

$$
\begin{aligned}
& \left(\ldots, b_{j+1}^{\prime} \otimes\left(b_{j+1} \circ((b \otimes \mathrm{id}) \oplus \mathrm{id})\right), b_{j}^{\prime} \otimes\left(\left(\mathrm{id} \otimes \bar{b}_{j}\right) \oplus \overline{\bar{b}}_{j}\right), b_{j-1}^{\prime} \otimes b_{j-1}\right) \\
& \quad=\left(\ldots,\left(b_{j+1}^{\prime} \otimes b_{j+1}\right) \circ((\mathrm{id} \otimes b \otimes \mathrm{id}) \oplus(\mathrm{id} \otimes \mathrm{id})),\left(b_{j}^{\prime} \otimes \mathrm{id} \otimes \bar{b}_{j}\right) \oplus\left(b_{j}^{\prime} \otimes \overline{\bar{b}}_{j}\right), b_{j-1}^{\prime} \otimes b_{j-1}, \ldots\right) \\
& \quad \stackrel{(7.1 .3)}{\sim}\left(\ldots, b_{j+1}^{\prime} \otimes b_{j+1},\left(b_{j}^{\prime} \otimes \mathrm{id} \otimes \bar{b}_{j}\right) \oplus\left(b_{j}^{\prime} \otimes \overline{\bar{b}}_{j}\right),((\mathrm{id} \otimes b \otimes \mathrm{id}) \oplus(\mathrm{id} \otimes \mathrm{id})) \circ\left(b_{j-1}^{\prime} \otimes b_{j-1}\right), \ldots\right) \\
& \left.\quad=\left(\ldots, b_{j+1}^{\prime} \otimes b_{j+1}, b_{j}^{\prime} \otimes\left(\left(\mathrm{id} \otimes \bar{b}_{j}\right) \oplus \overline{\bar{b}}_{j}\right), b_{j-1}^{\prime} \otimes((b \otimes \mathrm{id}) \oplus \mathrm{id}) \circ b_{j-1}\right), \ldots\right) .
\end{aligned}
$$

It is now straightforward to check that $\mathbf{B}$ is an $\mathbb{F}$-ring, $\psi^{i}: B^{i} \rightarrow \mathbf{B}$ are homomorphisms of $\mathbb{F}$-rings. If $f^{i}: B^{i} \rightarrow C$ are homomorphisms of $\mathbb{F}$-rings such that $f^{0} \circ \varphi^{0}=f^{1} \circ \varphi^{1}$, we define $f^{0} \odot f^{1}: \mathbf{B} \rightarrow C$ by

$$
f^{0} \odot f^{1}\left(\left(b_{l}, \ldots, b_{\delta}\right) / \sim\right)=f^{l}\left(b_{l}\right) \circ \cdots \circ f^{\delta}\left(b_{\delta}\right), \quad f^{j}=f^{j(\bmod 2)} .
$$

It is well defined: changing the representatives $\left(b_{l}, \ldots, b_{\delta}\right)$ by one of the moves (7.1.1), (7.1.2), (7.1.3) does not change the result of (7.1.7). For example, $f^{0} \odot f^{1}$ applied to both sides of (7.1.3) gives the same result

$$
\cdots \circ f^{j+1}\left(b_{j+1}\right) \circ\left[\left(f^{j+1}(b) \otimes f^{j}\left(\bar{b}_{j}\right)\right) \oplus f^{j}\left(\overline{\bar{b}}_{j}\right)\right] \circ f^{j-1}\left(b_{j-1}\right) \circ \cdots .
$$

The map $f^{0} \odot f^{1}$ is a homomorphism of $\mathbb{F}$-rings, and clearly it is the unique homomorphism such that

$$
\left(f^{0} \odot f^{1}\right) \circ \psi^{i}=f^{i}, \quad \text { i.e. } f^{0} \odot f^{1}\left(\left(b_{i}\right) / \sim\right)=f^{i}\left(b_{i}\right) \text { for } b_{i} \in B^{i} .
$$




\section{J. Shai Haran}

We write $B^{0} \odot{ }_{A} B^{1}$ for $\mathbf{B}, b_{l} \odot \cdots \odot b_{\delta}$ for the equivalence class $\left(b_{l} \ldots, b_{\delta}\right) / \sim$. Thus the arithmetic in $B^{0} \odot A B^{1}$ is governed by the three moves,

(i) $\cdots\left(b_{j+1} \circ \varphi^{j+1}(a)\right) \odot b_{j} \cdots=\cdots b_{j+1} \odot\left(\varphi^{j}(a) \circ b_{j}\right) \cdots \quad a \in A$,

(ii) $\cdots b_{j+1} \odot f \odot b_{j-1} \cdots=\cdots \odot\left(b_{j+1} \circ f \circ b_{j-1}\right) \odot \cdots \quad f \in \mathbb{F}$,

(iii) $\cdots b_{j+1} \circ((b \otimes \mathrm{id}) \oplus \mathrm{id}) \odot\left(\left(\mathrm{id} \otimes \bar{b}_{j}\right) \oplus \overline{\bar{b}}_{j}\right) \odot b_{j-1} \cdots=\cdots b_{j+1} \odot\left(\left(\mathrm{id} \otimes \bar{b}_{j}\right) \oplus \overline{\bar{b}}_{j}\right) \odot((b \otimes \mathrm{id}) \oplus \mathrm{id}) \circ b_{j-1} \cdots$.

This completes the proof of Theorem 7.1.

Remark 7.1.9. Note that we can consider $B^{i}$ as an $A$-module via $\varphi^{i}$, hence we have the tensor product $B^{0} \otimes_{A} B^{1}$; cf. Lemma 3.20. We have a map

$$
B^{0} \otimes_{A} B^{1} \rightarrow B^{0} \odot_{A} B^{1}, \quad b_{0} \otimes b_{1} \mapsto \psi^{0}\left(b_{0}\right) \otimes \psi^{1}\left(b_{1}\right)=\left(b_{0} \otimes \mathrm{id}\right) \odot\left(\mathrm{id} \otimes b_{1}\right),
$$

but unlike the case of commutative rings this is not an isomorphism.

Remark 7.1.10. A similar construction gives the fibred sum of $\mathbb{F}^{ \pm}$-rings or $\overline{\mathbb{F}}$-rings. If the $\mathbb{F}$ or $\mathbb{F}^{ \pm}$ or $\overline{\mathbb{F}}$-rings $A, B^{0}, B^{1}$ have compatible involutions, we get a well-defined involution on $B^{0} \odot B^{1}$ by

$$
\left(\left(b_{l}, \ldots, b_{\delta}\right) / \sim\right)^{\mathrm{t}}=\left(b_{\delta}^{\mathrm{t}}, \ldots, b_{l}^{\mathrm{t}}\right) / \sim
$$

(note that the moves $(7.1 .1),(7.1 .2),(7.1 .3)$ are all symmetric with respect to $\left.(\cdots)^{\mathrm{t}}\right)$. Hence also the categories $\mathbb{F}^{ \pm}-\mathcal{R}$ ings, $\mathbb{F}^{ \pm, t}-\mathcal{R}$ ings, $\overline{\mathbb{F}}$ - $\mathcal{R}$ ings, $\overline{\mathbb{F}}^{\mathrm{t}}$ - $\mathcal{R}$ ings have fibred sums. Similarly if $A, B^{0}, B^{1}$ have compatible $\mathbb{F}^{\lambda}$-structure, we get such a structure on $B^{0} \odot B^{1}$ via

$$
\wedge^{d}\left(\left(b_{l}, \ldots, b_{\delta}\right) / \sim\right)=\left(\wedge^{d} b_{l}, \ldots \wedge^{d} b_{\delta}\right) / \sim .
$$

\subsection{Fibred product of $\mathbb{F}$-schemes, the case of $\overline{\operatorname{Spec} \mathbb{Z}} \times \overline{\operatorname{Spec} \mathbb{Z}}$}

As a corollary of Theorem 7.1 we get the following results.

Theorem 7.2. The category $\mathcal{Z}$ ar- $\mathbb{F}-\mathcal{S}$ ch of Zariski $\mathbb{F}$-schemes has fibred products. Given maps in $\mathcal{Z}$ ar- $\mathbb{F}-\mathcal{S}$ ch $f^{0}: X^{0} \rightarrow Y, f^{1}: X^{1} \rightarrow Y$, there exist a Zariski $\mathbb{F}$-scheme

$$
X^{0} \prod_{Y} X^{1}
$$

and maps

$$
\pi^{j}: X^{0} \prod_{Y} X^{1} \rightarrow X^{j}, j=0,1,
$$

such that $f^{0} \circ \pi^{0}=f^{1} \circ \pi^{1}$, and for any Zariski $\mathbb{F}$-scheme $Z$, and any maps $g^{j}: Z \rightarrow X^{j}, j=0,1$, such that $f^{0} \circ g^{0}=f^{1} \circ g^{1}$ there exists a unique map

$$
g^{0} \Pi g^{1}: Z \rightarrow X^{0} \prod_{Y} X^{1}
$$

with $\pi^{j} \circ\left(g^{0} \Pi g^{1}\right)=g^{j}$ for $j=0,1$.

Proof. Write $Y=\bigcup_{i} \operatorname{Spec}\left(A_{i}\right),\left(f^{j}\right)^{-1}\left(\operatorname{Spec}\left(A_{i}\right)\right)=\bigcup_{k} \operatorname{Spec}\left(B_{i, k}^{j}\right), j=0,1$. Then $X^{0} \prod_{Y} X^{1}$ is obtained by gluing $\left\{\operatorname{Spec}\left(B_{i, k_{0}}^{0} \odot A_{i} B_{i, k_{1}}^{1}\right)\right\}_{i, k_{0}, k_{1}}$. For details, see, e.g., [Hart77, Theorem 3.3, p. 87].

As a corollary we obtain the following theorem.

Theorem 7.3. The category $\mathbb{F}$-Sch of $\mathbb{F}$-schemes has fibred products.

Proof. Given maps as in Definition 6.8,

$$
\varphi=\left\{\varphi_{i}^{j}\right\}_{j \geqslant \sigma(i)}: X=\left\{X_{j}\right\}_{J} \rightarrow Y=\left\{Y_{i}\right\}_{I}, \quad \varphi^{\prime}=\left\{\varphi_{i}^{\prime j^{\prime}}\right\}_{j^{\prime} \geqslant \sigma^{\prime}(i)}: X^{\prime}=\left\{X_{j^{\prime}}^{\prime}\right\}_{J^{\prime}} \rightarrow Y,
$$


the fibred product in $\mathbb{F}$-S $\mathcal{S}$ ch is clearly given by the inverse system $\left\{X_{j} \times_{Y_{i}} X_{j^{\prime}}^{\prime}\right\}$, the indexing set is $\left\{\left(j, j^{\prime}, i\right) \in J \times J^{\prime} \times I \mid j \geqslant \sigma(i), j^{\prime} \geqslant \sigma^{\prime}(i)\right\}$.

As an important example we have the arithmetical surface compactified

$$
\overline{\operatorname{Spec} \mathbb{Z}} \times \text { Spec } \mathbb{F} \overline{\operatorname{Spec} \mathbb{Z}},
$$

which is represented by $\left\{X_{N} \times_{\operatorname{Spec} \mathbb{F}} X_{M}\right\}$ with indexing set $\{(N, M) \in \mathbb{N} \times \mathbb{N} \mid N, M$ square-free $\}$, and with

$$
X_{N}=\operatorname{Spec} \mathbb{F}(\mathbb{Z}) \coprod_{\operatorname{Spec}(\mathbb{F}(\mathbb{Z}[1 / N]))} \operatorname{Spec}\left(\mathbb{F}\left(\mathbb{Z}\left[\frac{1}{N}\right]\right) \cap \mathcal{O}_{\mathbb{Q}, \eta}\right),
$$

as in Example 6.9. The $\mathbb{F}$-scheme

$$
\overline{\operatorname{Spec} \mathbb{Z}} \times \text { Spec } \mathbb{F} \overline{\operatorname{Spec} \mathbb{Z}}
$$

contains the open dense subset (which is a Zariski $\mathbb{F}$-scheme):

$$
\operatorname{Spec} \mathbb{F}(\mathbb{Z}) \times_{\text {Spec } \mathbb{F}} \operatorname{Spec} \mathbb{F}(\mathbb{Z})=\operatorname{Spec} \mathbb{F}(\mathbb{Z}) \odot_{\mathbb{F}} \mathbb{F}(\mathbb{Z}) .
$$

A basis for neighborhoods of $(p, \eta)$ is given by

$$
\mathbb{F}\left(\mathbb{Z}\left[\frac{1}{N}\right]\right) \odot_{\mathbb{F}} A_{M}
$$

where $p$ does not divide $N$, and $M$ is arbitrary (for the definition of $A_{M}$ see Example 6.9).

Similarly, for any number field $K$ we have the compactified surface

$$
\overline{\operatorname{Spec} \mathcal{O}_{K}} \times_{\operatorname{Spec} \mathbb{F}} \overline{\operatorname{Spec} \mathcal{O}_{K}} \text {. }
$$

It contains the open dense subset

$$
\operatorname{Spec} \mathbb{F}\left(\mathcal{O}_{K}\right) \bigcirc_{\mathbb{F}} \mathbb{F}\left(\mathcal{O}_{K}\right)
$$

\section{Monoids}

Since we defined $\mathbb{F}$-rings to be categories, and therefore have the operation of composition $\circ$, the resulting product (o) (actually the sum in $\mathbb{F}$-rings) is very complicated, resembling amalgams of groups, and it is difficult to calculate for specific examples. Unlike the classical theory, it does not reduce to the tensor product $\otimes$, cf. Remark 7.1.9. Similarly, the base change functor for a map of $\mathbb{F}$-rings $\varphi: A \rightarrow B, \varphi_{*}: A$-Mod $\rightarrow B$ - $\mathcal{M o d}, \varphi_{*} M=M_{B}$, is complicated, and again does not reduce to the tensor product $B \otimes_{A} M$, as it does in the classical theory, cf. Remark 3.23.11. In this section we shall give a softer theory that overcomes these difficulties by replacing everywhere $\mathbb{F}$-rings by $F$-monoids, which have composition $\circ$ only with elements of a fixed base $\mathbb{F}$-ring $F$. Since the constructions are repetitions of the constructions of earlier sections we will be more brief. We repeat the story up to the definition of Zariski $F$-monoid scheme. One can define $F$-monoid schemes to be the category of pro-objects of Zariski $F$-monoid-schemes, as we did in the context of $\mathbb{F}$-rings, cf. Definition 6.8, but one does not do it for several reasons. For instance, the basic Example 6.9 of the compactification of Spec $\mathbb{Z}$, and $\operatorname{Spec} \mathcal{O}_{K}$, does not go through in the context of $F$-monoids. As explained at the beginning of $\S 1$, working with $\overline{\operatorname{Spec} \mathbb{Z}}=\operatorname{Spec} \mathbb{Z} \cup\{\eta\}$ dictates taking $F=\mathbb{F}$, and since $\mathbb{F}$ does not have the vector $(1,1)$ addition is lost, cf. $(2.13 .4)$ : the functor $A \mapsto \mathbb{F}(A)$, from Rings to $\mathbb{F}$-monoids, is not fully faithful (compare with Example 1 of $\S 2.3$ ), and when we view $\mathbb{F}(A)$ as $\mathbb{F}$-monoid $\operatorname{Spec} \mathbb{F}(A)$ does not reduce to $\operatorname{Spec} A$. Thus for example viewing $\mathbb{F}(\mathbb{Z})$ as $\mathbb{F}$-monoid its spectrum has the cardinality of the continuum: for any set $S$ of primes we have the prime $\mathfrak{p}_{S} \in \operatorname{Spec} \mathbb{F}(\mathbb{Z}), \mathfrak{p}_{S}=\bigcup_{p \in S} p \cdot \mathbb{Z}$. 


\section{J. Shai HARAN}

On the other hand, using the localized version (Definition 8.20) of $\mathcal{O}_{X}$-monoids, one can use $\mathcal{O}_{X}$-monoids to define the flat (and étale) Grothendieck topologies on a given (Zariski) $\mathbb{F}$-scheme; see [TV05].

\subsection{F-monoids}

Definition 8.1. An $F$-monoid is a monoid object in the category of $F$-modules.

Thus an $F$-monoid $A=\left\{A_{Y, X}\right\}_{Y, X \in|\mathbb{F}|}$ has the operations

$$
\begin{aligned}
& F \text {-composition: } F_{Y^{\prime}, Y} \times A_{Y, X} \times F_{X, X^{\prime}} \rightarrow A_{Y^{\prime}, X^{\prime}}, \quad\left(f, a, f^{\prime}\right) \mapsto f \circ a \circ f^{\prime}, \\
& F \text {-tensor product: } F_{Y_{0}, X_{0}} \times A_{Y_{1}, X_{1}} \rightarrow A_{Y_{0} \otimes Y_{1}, X_{0} \otimes X_{1}}, \quad(f, a) \mapsto f \otimes a, \\
& \text { direct sum: } A_{Y_{0}, X_{0}} \times A_{Y_{1}, X_{1}} \rightarrow A_{Y_{0} \oplus Y_{1}, X_{0} \oplus X_{1}}, \quad\left(a_{0}, a_{1}\right) \mapsto a_{0} \oplus a_{1},
\end{aligned}
$$

satisfying the $F$-module axioms (3.1.4)-(3.1.15), together with the additional operation of 'tensor' product:

$$
A_{Y_{0}, X_{0}} \times A_{Y_{1}, X_{1}} \rightarrow A_{Y_{0} \otimes Y_{1}, X_{0} \otimes X_{1}}, \quad\left(a_{0}, a_{1}\right) \mapsto a_{0} \otimes a_{1} .
$$

This operation is bilinear over $F$ :

$$
\begin{gathered}
\left(a_{0} \oplus a_{0}^{\prime}\right) \otimes a_{1}=\left(a_{0} \otimes a_{1}\right) \oplus\left(a_{0}^{\prime} \otimes a_{1}\right), a_{0} \otimes\left(a_{1} \oplus a_{1}^{\prime}\right)=\left(a_{0} \otimes a_{1}\right) \oplus\left(a_{0} \otimes a_{1}^{\prime}\right), \quad a_{i}, a_{i}^{\prime} \in A \\
\left(f_{0} \circ a_{0} \circ f_{0}^{\prime}\right) \otimes\left(f_{1} \circ a_{1} \circ f_{1}^{\prime}\right)=\left(f_{0} \otimes f_{1}\right) \circ\left(a_{0} \otimes a_{1}\right) \circ\left(f_{0}^{\prime} \otimes f_{1}^{\prime}\right), \quad f_{i}, f_{i}^{\prime} \in F, a \in A, \\
\left(f \otimes a_{0}\right) \otimes a_{1}=f \otimes\left(a_{0} \otimes a_{1}\right)=a_{0} \otimes\left(f \otimes a_{1}\right), \quad f \in F, a_{i} \in A .
\end{gathered}
$$

It is also associative:

$$
a_{0} \otimes\left(a_{1} \otimes a_{2}\right)=\left(a_{0} \otimes a_{1}\right) \otimes a_{2}, \quad a_{i} \in A,
$$

commutative:

$$
a_{0} \otimes a_{1}=a_{1} \otimes a_{0}, \quad a_{i} \in A,
$$

and unital:

$$
\text { there exists (a unique) } 1=1_{A} \in A_{[1],[1]} \text { with } 1 \otimes a=a \text {, for all } a \in A \text {. }
$$

A map of $F$-monoids $\varphi: A \rightarrow A^{\prime}$ is a map of $F$-modules respecting $\otimes$ and 1 , i.e. we have

$$
\varphi_{Y, X}: A_{Y, X} \rightarrow A_{Y, X}^{\prime}, \quad Y, X \in|\mathbb{F}|,
$$

satisfying

$$
\begin{gathered}
\varphi\left(f \circ a \circ f^{\prime}\right)=f \circ \varphi(a) \circ f^{\prime}, \quad f, f^{\prime} \in F, a \in A, \\
\varphi(f \otimes a)=f \otimes \varphi(a), \quad f \in F, a \in A, \\
\varphi\left(a_{0} \oplus a_{1}\right)=\varphi\left(a_{0}\right) \oplus \varphi\left(a_{1}\right), \quad a_{i} \in A, \\
\varphi\left(a_{0} \otimes a_{1}\right)=\varphi\left(a_{0}\right) \otimes \varphi\left(a_{1}\right), \quad a_{i} \in A, \\
\varphi\left(1_{A}\right)=1_{A^{\prime}} .
\end{gathered}
$$

Thus we have the category $F$-Mon of $F$-monoids; it is a subcategory of F-Mod. The $F$-module $F$ itself, considered as an $F$-monoid, is the initial object of $F$-Mon: for any $F$-monoid $A$ we have the map $F \rightarrow A, f \mapsto f \otimes 1_{A}$.

All our examples of $\mathbb{F}$-rings are of course $\mathbb{F}$-monoids. Note that the functor from commutative rings to $\mathbb{F}$-monoids, $A \mapsto \mathbb{F}(A)$, is faithful, but is not fully faithful: there are more maps $\mathbb{F}(A) \rightarrow \mathbb{F}(B)$ in $\mathbb{F}$-Mon than there are maps $A \rightarrow B$ in $\mathcal{R}$ ing. On the other hand, we can consider this functor as taking values in $\mathbb{F}(\mathbb{Z})$-Mon, or $\mathbb{S}-\mathcal{M}$ on, or $\mathbb{S}^{\text {op }}$-Mon, cf. Example 3 (2.15.1), in which case it is fully faithful. 


\section{NON-ADDITIVE GEOMETRY}

The category of $F$-monoids has fibred product $A^{0} \prod_{B} A^{1}$, arbitrary products $\prod_{i} A^{i}$, and arbitrary inverse limits $\lim _{i} A^{i}$.

Definition 8.3. For an $F$-monoid $A$, an equivalence ideal $\mathcal{E}$ is a collection of subsets

$$
\mathcal{E}=\coprod_{Y, X \in|\mathbb{F}|} \mathcal{E}_{Y, X}, \quad \mathcal{E}_{Y, X} \subset A_{Y, X} \times A_{Y, X},
$$

such that $\mathcal{E}_{Y, X}$ is an equivalence relation on $A_{Y, X}$, and $\mathcal{E}$ is an $A$-submodule of $A \prod A$, i.e. it is an $F$-submodule:

$$
\begin{gathered}
\left(a_{0}, a_{1}\right) \in \mathcal{E}_{Y, X}, \quad f \in F_{Y^{\prime}, Y}, \quad f^{\prime} \in F_{X, X^{\prime}} \Rightarrow\left(f \circ a_{0} \circ f^{\prime}, f \circ a_{1} \circ f^{\prime}\right) \in \mathcal{E}_{Y^{\prime}, X^{\prime}}, \\
\left(a, a^{\prime}\right) \in \mathcal{E}_{Y, X}, \quad f \in F_{Y_{0}, X_{0}} \Rightarrow\left(f \otimes a, f \otimes a^{\prime}\right) \in \mathcal{E}_{Y_{0} \otimes Y, X_{0} \otimes X}, \\
\left(a_{0}, a_{0}^{\prime}\right) \in \mathcal{E}_{Y_{0}, X_{0}}, \quad\left(a_{1}, a_{1}^{\prime}\right) \in \mathcal{E}_{Y_{1}, X_{1}} \Rightarrow\left(a_{0} \oplus a_{1}, a_{0}^{\prime} \oplus a_{1}^{\prime}\right) \in \mathcal{E}_{Y_{0} \oplus Y_{1}, X_{0} \oplus X_{1}},
\end{gathered}
$$

and moreover it is closed under $A$-tensor product:

$$
\left(a, a^{\prime}\right) \in \mathcal{E}_{Y, X}, \quad a_{0} \in A_{Y_{0}, X_{0}} \Rightarrow\left(a_{0} \otimes a, a_{0} \otimes a^{\prime}\right) \in \mathcal{E}_{Y_{0} \otimes Y, X_{0} \otimes X} .
$$

(Note that (8.3.3) follows from (8.3.5) by taking $a_{0}=f \otimes 1_{A}$.)

Given an equivalence ideal $\mathcal{E}$ of $A$ we can form the quotient

$$
A / \mathcal{E}=\coprod_{Y, X \in|\mathbb{F}|} A_{Y, X} / \mathcal{E}_{Y, X},
$$

which has the structure of $F$-monoid such that the canonical projection $\pi: A \rightarrow A / \mathcal{E}$ is a homomorphism of $F$-monoids. For a map $\varphi: A \rightarrow B$ in $F$-Mon we have the equivalence ideal of $A$, $\mathcal{K} \mathcal{E R}(\varphi)=\coprod_{Y, X} \mathcal{K} \mathcal{E} \mathcal{R}(\varphi)_{Y, X}$,

$$
\mathcal{K} \mathcal{E} \mathcal{R}(\varphi)_{Y, X}=\left\{\left(a, a^{\prime}\right) \in A_{Y, X} \times A_{Y, X} \mid \varphi(a)=\varphi\left(a^{\prime}\right)\right\}
$$

and $\varphi$ factorizes as epimorphism $\pi$ followed by an injection $\bar{\varphi}$, as in the diagram.

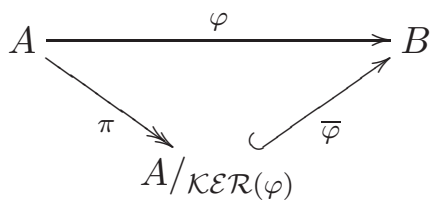

\subsection{Modules over an $\boldsymbol{F}$-monoid}

Definition 8.5. For an $F$-monoid $A$, an $A$-module $M$ is an $F$-module together with maps

$$
A_{Y_{0}, X_{0}} \times M_{Y_{1}, X_{1}} \rightarrow M_{Y_{0} \otimes Y_{1}, X_{0} \otimes X_{1}}, \quad(a, m) \mapsto a \otimes m,
$$

which are bilinear over $F$ : for $f, f_{i}, f_{i}^{\prime} \in F, a, a_{i} \in A, m, m_{i} \in M$,

$$
\begin{gathered}
\left(f_{1} \circ a \circ f_{1}^{\prime}\right) \otimes\left(f_{2} \circ m \circ f_{2}^{\prime}\right)=\left(f_{1} \otimes f_{2}\right) \circ(a \otimes m) \circ\left(f_{1}^{\prime} \otimes f_{2}^{\prime}\right), \\
(f \otimes a) \otimes m=f \otimes(a \otimes m)=a \otimes(f \otimes m), \\
\left(a_{0} \oplus a_{1}\right) \otimes m=\left(a_{0} \otimes m\right) \oplus\left(a_{1} \otimes m\right), a \otimes\left(m_{0} \oplus m_{1}\right)=\left(a \otimes m_{0}\right) \oplus\left(a \otimes m_{1}\right),
\end{gathered}
$$

associative:

$$
a_{0} \otimes\left(a_{1} \otimes m\right)=\left(a_{0} \otimes a_{1}\right) \otimes m,
$$

and unital:

$$
1_{A} \otimes m=m .
$$

(Note that the first equality in (8.5.2) follows from (8.5.4) and (8.5.5) by taking $a_{0}=f \otimes 1_{A}$.) 


\section{J. Shai HARAN}

The above defines a 'left' $A$-module; we can similarly define a 'right' $A$-module, but these notions are equivalent: any left $A$-module $M$ can be made into a right $A$-module by putting

$$
m \otimes a \stackrel{\text { def }}{=} c_{W, Y}^{*} \circ(a \otimes m) \circ c_{X, Z}^{*} \text { for } m \in M_{Y, X}, a \in A_{W, Z}\left(c^{*}\right. \text { as in (1.16)). }
$$

Similarly any right $A$-module can be made into a left $A$-module; hence we shall not distinguish between left and right $A$-modules.

Definition 8.6. A map of $A$-modules $\varphi: M \rightarrow M^{\prime}$ is a map of $F$-modules respecting $\otimes$, i.e. we have

$$
\begin{gathered}
\varphi_{Y, X}: M_{Y, X} \rightarrow M_{Y, X}^{\prime}, \quad Y, X \in|\mathbb{F}|, \\
\varphi\left(f \circ m \circ f^{\prime}\right)=f \circ \varphi(m) \circ f^{\prime}, \quad f, f^{\prime} \in F, \\
\varphi(f \otimes m)=f \otimes \varphi(m), \quad f \in F, \\
\varphi\left(m_{0} \oplus m_{1}\right)=\varphi\left(m_{0}\right) \oplus \varphi\left(m_{1}\right), \quad m_{i} \in M, \\
\varphi(a \otimes m)=a \otimes \varphi(m), \quad a \in A .
\end{gathered}
$$

(Note that (8.6.3) follows from (8.6.5).)

Thus we have a category $A_{F-}-\mathcal{M o d}$ of $A$-modules; it is a subcategory of $F$-Mod. Note that if $A$ is an $\mathbb{F}$-ring, we can consider $A_{\mathbb{F}}:=A$ as an $\mathbb{F}$-monoid, and $A$-Mod $\subseteq A_{\mathbb{F}}-\mathcal{M}$ od. On the other hand we can consider $A_{A}:=A$ as an $A$-monoid, and then $A$-Mod $=A_{A}$-Mod. The category of $A$-modules has the initial and final object $0=\left\{0_{Y, X}\right\}_{Y, X \in|\mathbb{F}|}$. One defines the notion of an $A$-submodule $M_{0} \subseteq M$ in the evident way; an $A$-submodule of $A$ is called an ideal.

The category $A_{F}-\mathcal{M} o d$ has fibred product

$$
\left(M^{0} \prod_{M} M^{1}\right)_{Y, X}=\left\{\left(m_{0}, m_{1}\right) \in M_{Y, X}^{0} \times M_{Y, X}^{1} \mid \varphi_{0}\left(m_{0}\right)=\varphi_{1}\left(m_{1}\right)\right\} \quad \text { for } \varphi_{i}: M^{i} \rightarrow M ;
$$

it has arbitrary products $\prod_{i} M^{i}$, and arbitrary inverse limits $\varliminf_{i} M^{i}$,

$$
\lim _{i} M^{i}=\left\{\left(m_{i}\right) \in \prod_{i} M^{i} \mid \varphi_{i^{\prime}, i}\left(m_{i}\right)=m_{i^{\prime}}\right\} .
$$

For an $F$-monoid $A$, and $A$-module $M$, an equivalence $A$-module of $M$ is an $A$-submodule $\mathcal{E}$ of $M \prod M$, such that $\mathcal{E}_{Y, X}$ is an equivalence relation on $M_{Y, X}$. We can form the quotient

$$
M / \mathcal{E}=\coprod_{Y, X \in|\mathbb{F}|}(M / \mathcal{E})_{Y, X}, \quad(M / \mathcal{E})_{Y, X}=M_{Y, X} / \mathcal{E}_{Y, X},
$$

and it has the structure of an $A$-module such that the canonical projection $\pi: M \rightarrow M / \mathcal{E}$ is a homomorphism of $A$-modules. For a map $\varphi: M \rightarrow N$ in $A$-Mod we have the equivalence $A$-module of $M, \mathcal{K E \mathcal { R }}(\varphi)=M \prod_{N} M$, and $\varphi$ factorizes as an epimorphism $\pi$ followed by an injection $\bar{\varphi}$, as in the diagram.

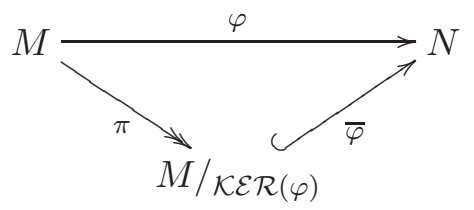

For a map $\varphi: M \rightarrow N$ in $A$ - $\mathcal{M o d}$ we have the $A$-submodule $M$,

$$
\varphi^{-1}(0)=\{m \in M \mid \varphi(m)=0\},
$$

which is the kernel of $\varphi$ in $A$-Mod; we have also the $A$-submodule of $N$,

$$
\varphi(M)=\{\varphi(m) \mid m \in M\} .
$$


For an equivalence $A$-module $\mathcal{E}$ of $M$, we let $Z(\mathcal{E})$ denote $\pi^{-1}(0), \pi: M \rightarrow M / \mathcal{E}$ the projection

$$
Z(\mathcal{E})=\pi^{-1}(0)=\{m \in M \mid(m, 0) \in \mathcal{E}\}
$$

For an $A$-submodule $M_{0} \subseteq M$ we let $E\left(M_{0}\right)$ be the equivalence $A$-module of $M$ generated by $M_{0} \times\{0\}$. We write $M / M_{0}$ for $M / E\left(M_{0}\right)$. For a map $\varphi: M \rightarrow N$ in $A$-Mod we have its cokernel

$$
\text { Coker } \varphi=N / \varphi(M)=N / E(\varphi(M)) \text {. }
$$

We have for $A$-submodules $M_{i}$ of $M$, and equivalence $A$-modules $\mathcal{E}_{i}$ of $M$,

$$
\begin{gathered}
M_{0} \subseteq M_{1} \Rightarrow E\left(M_{0}\right) \subseteq E\left(M_{1}\right), \\
\mathcal{E}_{0} \subseteq \mathcal{E}_{1} \Rightarrow Z\left(\mathcal{E}_{0}\right) \subseteq Z\left(\mathcal{E}_{1}\right), \\
M_{0} \subseteq Z E\left(M_{0}\right), \\
\mathcal{E}_{0} \supseteq E Z\left(\mathcal{E}_{0}\right), \\
E\left(M_{0}\right)=E Z E\left(M_{0}\right), \\
Z\left(\mathcal{E}_{0}\right)=Z E Z\left(\mathcal{E}_{0}\right) .
\end{gathered}
$$

We have a bijection between the set

$$
\left\{Z\left(\mathcal{E}_{0}\right) \mid \mathcal{E}_{0} \subseteq M \prod M\right\} \equiv\left\{M_{0} \subseteq M \mid M_{0}=Z E\left(M_{0}\right)\right\}
$$

and the set

$$
\left\{E\left(M_{0}\right) \mid M_{0} \subseteq M\right\} \equiv\left\{\mathcal{E}_{0} \mid \mathcal{E}_{0}=E Z\left(\mathcal{E}_{0}\right)\right\}
$$

given by

$$
M_{0} \longmapsto E\left(M_{0}\right), \quad Z\left(\mathcal{E}_{0}\right) \longleftrightarrow \mathcal{E}_{0} .
$$

Lemma 3.13 and its Corollary 3.14 remain valid: the equivalence $A$-module of $M$ generated by the $A$-submodule $M_{0} \subseteq M, \mathcal{E}=E\left(M_{0}\right)$, can be described explicitly:

$$
\begin{aligned}
\mathcal{E}_{Y, X}=\{ & \left(m, m^{\prime}\right) \in M_{Y, X} \times M_{Y, X} \mid \exists \text { path } m=m_{0}, m_{1}, \ldots, m_{l}=m^{\prime}, \\
& \text { with }\left\{m_{j}, m_{j+1}\right\} \text { of the form }\left\{f_{j} \circ\left(\widetilde{m}_{j} \oplus n_{j}\right) \circ f_{j}^{\prime}, f_{j} \circ\left(\widetilde{m}_{j} \oplus 0\right) \circ f_{j}^{\prime}\right\}, \\
& \left.f_{j}, f_{j}^{\prime} \in F, \widetilde{m}_{j} \in M, n_{j} \in M_{0}\right\} .
\end{aligned}
$$

We have $M_{0}=Z E\left(M_{0}\right)$ if and only if for all $f, f^{\prime} \in F, m \in M, m_{0} \in M_{0}$,

$$
f \circ\left(m \oplus m_{0}\right) \circ f^{\prime} \in M_{0} \Leftrightarrow f \circ(m \oplus 0) \circ f^{\prime} \in M_{0} .
$$

For $A$-submodules $M_{i}$ of $M$, we have their intersection $\bigcap_{i} M_{i} \subseteq M$, and their sum

$$
\sum M_{i}=\left\{f \circ\left(\bigoplus_{i} m_{i}\right) \circ f^{\prime} \mid f, f^{\prime} \in F, m_{i} \in M_{i}\right\} .
$$

The $A$-submodule generated by a subset $\left\{m_{i}\right\}_{i \in I} \subseteq M$ is described explicitly as

$$
\sum A m_{i} \stackrel{\text { def }}{=}\left\{f \circ\left(\bigoplus_{i}\left(a_{i} \otimes m_{i}\right)\right) \circ f^{\prime} \mid f, f^{\prime} \in F, a_{i} \in A\right\} .
$$

Given an $A$-module $M$, and an ideal $\mathfrak{a} \subseteq A$, we have the $A$-submodule of $M$,

$$
\mathfrak{a} \cdot M=\left\{f \circ\left(\bigoplus_{i}\left(a_{i} \otimes m_{i}\right)\right) \circ f^{\prime} \mid f, f^{\prime} \in F, a_{i} \in \mathfrak{a}\right\} .
$$

Given $A$-submodules $M_{0}, M_{1} \subseteq M$, we have the ideal of A,

$$
\left(M_{0}: M_{1}\right)=\left\{a \in A \mid a \otimes m \in M_{0} \forall m \in M_{1}\right\} .
$$




\section{J. SHAi HARAN}

\subsection{Functorial operations on modules}

Sums and direct limits. Given a collection of $A$-modules $\left\{M_{i}\right\}_{i \in I}$, we have their sum in the category $A-\mathcal{M o d}$ (cf. Proposition 3.17):

$$
\begin{gathered}
\left(\coprod_{i \in I} M_{i}\right)_{Y, X}=\left\{\left(f,\left\{m_{i}\right\}_{i \in I_{0}}, f^{\prime}\right) \mid f \in F_{Y, \oplus_{i \in I_{0}} Y_{i}}, f^{\prime} \in F_{\oplus_{i \in I_{0}} X_{i}, X},\right. \\
\left.m_{i} \in\left(M_{i}\right)_{Y_{i}, X_{i}}, I_{0} \subseteq I \text { a finite subset }\right\} / \sim
\end{gathered}
$$

Here $\sim$ is the equivalence relation generated by

$$
\begin{gathered}
\left(f \circ\left(\bigoplus_{i \in I_{0}} f_{i}\right),\left\{m_{i}\right\}_{i \in I_{0}},\left(\bigoplus_{i \in I_{0}} f_{i}^{\prime}\right) \circ f^{\prime}\right) \sim\left(f,\left\{f_{i} \circ m_{i} \circ f_{i}^{\prime}\right\}_{i \in I_{0}}, f^{\prime}\right), \\
\left(f,\left\{m_{i}\right\}_{i \in I_{0}}, f^{\prime}\right) \sim\left(f,\left\{m_{i}\right\}_{i \in I_{0}} \cup\left\{\operatorname{id}_{[0]}\right\}, f^{\prime}\right) .
\end{gathered}
$$

The structure of $A$-module on $\coprod_{i \in I} M_{i}$ is defined by:

F-composition

$$
g \circ\left(f,\left\{m_{i}\right\}, f^{\prime}\right) / \sim \circ g^{\prime}=\left(g \circ f,\left\{m_{i}\right\}, f^{\prime} \circ g^{\prime}\right) / \sim
$$

sum

$$
\left(f_{0},\left\{m_{i}\right\}_{i \in I_{0}}, f_{0}^{\prime}\right) / \sim \oplus\left(f_{1},\left\{m_{i}^{\prime}\right\}_{i \in I_{1}}, f_{1}^{\prime}\right)=\left(f_{0} \oplus f_{1},\left\{\widetilde{m}_{i}\right\}_{i \in I_{0} \cup I_{1}}, f_{0}^{\prime} \oplus f_{1}^{\prime}\right) / \sim
$$

with $\widetilde{m}_{i}=m_{i}$ (respectively $m_{i}^{\prime}, m_{i} \oplus m_{i}^{\prime}$ ) for $i \in I_{0} \backslash I_{1}$ (respectively $i \in I_{1} \backslash I_{0}, i \in I_{0} \cap I_{1}$ ); and $A$-tensor product

$$
a \otimes\left(f,\left\{m_{i}\right\}_{i \in I_{0}}, f^{\prime}\right) / \sim=\left(\operatorname{id}_{W} \otimes f,\left\{a \otimes m_{i}\right\}_{i \in I_{0}}, \operatorname{id}_{Z} \otimes f^{\prime}\right) / \sim, \quad a \in A_{W, Z} .
$$

Note that for $g \in F_{W, Z}$ :

$$
\begin{aligned}
g \otimes\left(f,\left\{m_{i}\right\}_{i \in I_{0}}, f^{\prime}\right) / \sim & =\left(g \otimes 1_{A}\right) \otimes\left(f,\left\{m_{i}\right\}_{i \in I_{0}}, f^{\prime}\right) / \sim \\
& =\left(\operatorname{id}_{W} \otimes f,\left\{g \otimes m_{i}\right\}_{i \in I_{0}}, \mathrm{id}_{Z} \otimes f^{\prime}\right) / \sim \\
& =\left(\operatorname{id}_{W} \otimes f,\left\{\left(g \otimes \mathrm{id}_{Y_{i}}\right) \circ\left(\mathrm{id}_{Z} \otimes m_{i}\right)\right\}_{i \in I_{0}}, \mathrm{id}_{Z} \otimes f^{\prime}\right) / \sim, \\
\text { or } & =\left(\operatorname{id}_{W} \otimes f,\left\{\left(\operatorname{id}_{W} \otimes m_{i}\right) \circ\left(g \otimes \operatorname{id}_{X_{i}}\right)\right\}_{i \in I_{0}}, \operatorname{id}_{Z} \otimes f^{\prime}\right) / \sim \\
& =\left(\left(\operatorname{id}_{W} \otimes f\right) \circ \bigoplus_{I_{0}}\left(g \otimes \operatorname{id}_{Y_{i}}\right),\left\{\operatorname{id}_{Z} \otimes m_{i}\right\}_{i \in I_{0}}, \operatorname{id}_{Z} \otimes f^{\prime}\right) /, \\
\text { or } & =\left(\operatorname{id}_{W} \otimes f,\left\{\operatorname{id}_{W} \otimes m_{i}\right\}_{i \in I_{0}}, \bigoplus_{I_{0}}\left(g \otimes \operatorname{id}_{X_{i}}\right) \circ\left(\operatorname{id}_{Z} \otimes f^{\prime}\right)\right) / \\
& =\left(g \otimes f,\left\{\operatorname{id}_{Z} \otimes m_{i}\right\}_{i \in I_{0}}, \operatorname{id}_{Z} \otimes f^{\prime}\right) / \sim, \\
\text { or } & =\left(\operatorname{id}_{W} \otimes f,\left\{\operatorname{id}_{W} \otimes m_{i}\right\}_{i \in I_{0}}, g \otimes f^{\prime}\right) / \sim .
\end{aligned}
$$

We write $f \circ\left(\bigoplus_{I_{0}} m_{i}\right) \circ f^{\prime}$ for $\left(f,\left\{m_{i}\right\}_{i \in I_{0}}, f^{\prime}\right) / \sim$.

Given a functor $i \mapsto M_{i}$ from a small category $I$ to $A$-Mod, we have the direct limit

$$
\underset{I}{\lim _{I}} M_{i}=\coprod_{I} M_{i} / \mathcal{E}
$$

where $\mathcal{E}$ is the equivalence $A$-module of $\coprod_{I} M_{i}$ generated by

$$
\left\{\left(\operatorname{id}_{Y} \circ\left(m_{i}\right) \circ \operatorname{id}_{X}, \operatorname{id}_{Y} \circ\left(\varphi_{j i}\left(m_{i}\right)\right) \circ \operatorname{id}_{X}\right) \mid m_{i} \in M_{i}, \varphi_{j i}: M_{i} \rightarrow M_{j}\right\} .
$$

In particular we have the push-out $M_{0} \coprod_{M} M_{1}$ for homomorphisms $\psi_{i}: M \rightarrow M_{i}$,

$$
M_{0} \coprod_{M} M_{1}=\left(M_{0} \coprod M_{1}\right) / \mathcal{E}
$$




\section{NON-ADDITIVE GEOMETRY}

where $\mathcal{E}$ is the equivalence $A$-module of $M_{0} \coprod M_{1}$ generated by

$$
\left\{\left(f \circ\left(\psi_{0}(m)\right) \circ f^{\prime}, f \circ\left(\psi_{1}(m)\right) \circ f^{\prime}\right) \mid m \in M\right\} .
$$

Free modules. Let $\mathfrak{s}$ be a formal symbol representing a map $X_{0} \rightarrow Y_{0}, X_{0}, Y_{0} \in|\mathbb{F}|$. The free $A$-module on $\mathfrak{s}$ is

$$
(A \cdot \mathfrak{s})_{Y, X}=\left\{\left(f, a, f^{\prime}\right) \mid f \in F_{Y, W \oplus Y_{0}}, f^{\prime} \in F_{Z \oplus X_{0}, X}, a \in A_{W, Z}\right\} / \sim
$$

where $\sim$ is the equivalence relation generated by

$$
\left(f \circ\left(g \otimes \operatorname{id}_{Y_{0}}\right), a,\left(g^{\prime} \otimes \operatorname{id}_{X_{0}}\right) \circ f^{\prime}\right) \sim\left(f, g \circ a \circ g^{\prime}, f^{\prime}\right), \quad f, f^{\prime}, g, g^{\prime} \in F .
$$

The structure of $A$-module on $A \cdot \mathfrak{s}$ is defined by

$$
\begin{aligned}
& g \circ\left(f, a, f^{\prime}\right) / \sim \circ g^{\prime}=\left(g \circ f, a, f^{\prime} \circ g^{\prime}\right) / \sim, \quad f, f^{\prime}, g, g^{\prime} \in F, \\
&\left(f_{0}, a_{0}, f_{0}^{\prime}\right) / \sim \oplus\left(f_{1}, a_{1}, f_{1}^{\prime}\right) / \sim=\left(f_{0} \oplus f_{0}^{\prime}, a_{0} \oplus a_{1}, f_{0}^{\prime} \oplus f_{1}^{\prime}\right), \quad f_{i}, f_{i}^{\prime} \in F, \\
& a_{0} \otimes\left(f, a, f^{\prime}\right) / \sim=\left(\operatorname{id}_{W} \otimes f, a_{0} \otimes a, \mathrm{id}_{Z} \otimes f^{\prime}\right) / \sim, \quad a_{0} \in A_{W, Z} .
\end{aligned}
$$

We write $f \circ(a \otimes \mathfrak{s}) \circ f^{\prime}$ for $\left(f, a, f^{\prime}\right) / \sim$. Note that for $g \in F_{W, Z}$,

$$
\begin{aligned}
g \otimes\left(f \circ(a \otimes \mathfrak{s}) \circ f^{\prime}\right) & \stackrel{\text { def }}{=}\left(g \otimes 1_{A}\right) \otimes\left(f \circ(a \otimes \mathfrak{s}) \circ f^{\prime}\right) \\
& =\left(\mathrm{id}_{W} \otimes f\right) \circ(g \otimes a \otimes \mathfrak{s}) \circ\left(\mathrm{id}_{Z} \otimes f^{\prime}\right) \\
& =(g \otimes f) \circ\left(\mathrm{id}_{Z} \otimes a \otimes \mathfrak{s}\right) \circ\left(\mathrm{id}_{Z} \otimes f^{\prime}\right) \\
& =\left(\mathrm{id}_{W} \otimes f\right) \circ\left(\mathrm{id}_{W} \otimes a \otimes \mathfrak{s}\right) \circ\left(g \otimes f^{\prime}\right) .
\end{aligned}
$$

For a set $\mathcal{S}=\left\{\mathfrak{s}_{i}\right\}_{i \in I}$ over $|\mathbb{F}| \times|\mathbb{F}|$, with $\mathfrak{s}_{i} \mapsto\left(Y_{i}, X_{i}\right)$, the free $A$-module on $\mathcal{S}$ is the sum

$$
A \cdot \mathcal{S}=\coprod_{i \in I} A \cdot \mathfrak{s}_{i}
$$

or explicitly,

$$
(A \cdot \mathcal{S})_{Y, X}=\left\{\left(f,\left\{a_{i}\right\}_{i \in I_{0}}, f^{\prime}\right) \mid f \in F_{Y, \oplus_{I_{0}}}\left(W_{i} \otimes Y_{i}\right), f^{\prime} \in F_{\oplus_{I_{0}}}\left(Z_{i} \otimes X_{i}\right), X, a_{i} \in A_{W_{i}, Z_{i}}, I_{0} \subseteq I \text { finite }\right\} / \sim
$$

with the equivalence relation $\sim$ generated by

$$
\begin{gathered}
\left(f \circ\left(\bigoplus_{I_{0}}\left(g_{i} \otimes \operatorname{id}_{Y_{i}}\right)\right),\left\{a_{i}\right\}_{i \in I_{0}},\left(\bigoplus_{I_{0}}\left(g_{i}^{\prime} \otimes \operatorname{id}_{X_{i}}\right)\right) \circ f^{\prime}\right) \sim\left(f,\left\{g_{i} \circ a_{i} \circ g_{i}^{\prime}\right\}_{i \in I_{0}}, f^{\prime}\right), f, f^{\prime}, g_{i}, g_{i}^{\prime} \in F, \\
\left(f,\left\{a_{i}\right\}_{i \in I_{0}}, f^{\prime}\right) \sim\left(f,\left\{a_{i}\right\}_{i \in I_{0}} \cup\left\{\operatorname{id}_{[0]}\right\}, f^{\prime}\right) .
\end{gathered}
$$

We write $f \circ\left(\bigoplus_{I_{0}}\left(a_{i} \otimes \mathfrak{s}_{i}\right)\right) \circ f^{\prime}$ for $\left(f,\left\{a_{i}\right\}_{i \in I_{0}}, f^{\prime}\right) / \sim$. The functor $\mathcal{S} \mapsto A \cdot \mathcal{S}$ from sets over $|\mathbb{F}| \times|\mathbb{F}|$ to $A$-Mod is left-adjoint to the forgetful functor $M \mapsto \coprod_{Y, X} M_{Y, X}$,

$$
\operatorname{Hom}_{A-\mathcal{M} o d}(A \cdot \mathcal{S}, M)=\left\{\left(m_{i}\right)_{i \in I} \mid m_{i} \in M_{Y_{i}, X_{i}}\right\}=\operatorname{Hom}_{\mathcal{S} e t s /|\mathbb{F}| \times|\mathbb{F}|}(\mathcal{S}, M) .
$$

Tensor products. For $M_{0}, M_{1}, N \in A$-Mod, we let $\mathcal{B} i l i n_{F}^{A}\left(M_{0}, M_{1} ; N\right)$ denote the maps $\varphi$ : $M_{0} \times M_{1} \rightarrow N$ satisfying ' $A_{F}$-bilinearity':

$$
\begin{gathered}
\varphi\left(\left(M_{0}\right)_{Y_{0}, X_{0}} \times\left(M_{1}\right)_{Y_{1}, X_{1}}\right) \subseteq N_{Y_{0} \otimes Y_{1}, X_{0} \otimes X_{1}}, \\
\varphi\left(m_{0} \oplus m_{0}^{\prime}, m_{1}\right)=\varphi\left(m_{0}, m_{1}\right) \oplus \varphi\left(m_{0}^{\prime}, m_{1}\right), \\
\varphi\left(m_{0}, m_{1} \oplus m_{1}^{\prime}\right)=\varphi\left(m_{0}, m_{1}\right) \oplus \varphi\left(m_{0}, m_{1}^{\prime}\right), \quad m_{i}, m_{i}^{\prime} \in M_{i}, \\
\varphi\left(a \otimes m_{0}, m_{1}\right)=a \otimes \varphi\left(m_{0}, m_{1}\right)=\varphi\left(m_{0}, a \otimes m_{1}\right), \quad m_{i} \in M_{i}, a \in A, \\
\varphi\left(g_{0} \circ m_{0} \circ g_{0}^{\prime}, g_{1} \circ m_{1} \circ g_{1}^{\prime}\right)=\left(g_{0} \otimes g_{1}\right) \circ \varphi\left(m_{0}, m_{1}\right) \circ\left(g_{0}^{\prime} \otimes g_{1}^{\prime}\right) \quad g_{i}, g_{i}^{\prime} \in F .
\end{gathered}
$$




\section{J. Shai Haran}

The free $A$-module on the set $\left\{\varphi\left(m_{0}, m_{1}\right)\right\}_{m_{i} \in M_{i}}$, divided by the equivalence $A$-module generated by $(8.15 .2),(8.15 .3),(8.15 .4)$, is denoted by $M_{0} \otimes_{A / F} M_{1}$, and the map

$$
M_{0} \times M_{1} \rightarrow M_{0} \otimes_{A / F} M_{1},\left(m_{0}, m_{1}\right) \mapsto m_{0} \otimes m_{1}=\text { image of } \varphi\left(m_{0} \cdot m_{1}\right)
$$

is the universal bilinear map:

$$
\mathcal{B i l i n}_{F}^{A}\left(M_{0}, M_{1} ; N\right)=\operatorname{Hom}_{A-\mathcal{M o d}}\left(M_{0} \otimes_{A / F} M_{1}, N\right) .
$$

We can describe $M_{0} \otimes_{A} M_{1}$ quite explicitly as

$$
\begin{gathered}
\left(M_{0} \otimes_{A} M_{1}\right)_{Y, X}=\left\{\left(f,\left\{m_{0}^{i}\right\}_{i \in I},\left\{m_{1}^{i}\right\}_{i \in I}, g\right) \mid f \in F_{Y, \oplus_{I}\left(Y_{0}^{i} \otimes Y_{1}^{i}\right)}, g \in F_{\bigoplus_{I}\left(X_{0}^{I} \otimes X_{1}^{i}\right), X},\right. \\
\left.m_{0}^{i} \in\left(M_{0}\right)_{Y_{0}^{i}, X_{0}^{i}}, m_{1}^{i} \in\left(M_{1}\right)_{Y_{1}^{i}, X_{1}^{i}}, I \in|\mathbb{F}|\right\} / \sim
\end{gathered}
$$

where the equivalence relation $\sim$ is generated by

$$
\begin{gathered}
\left(f \circ\left(\bigoplus_{I}\left(f_{0}^{i} \otimes f_{1}^{i}\right)\right),\left\{m_{0}^{i}\right\}_{I},\left\{m_{1}^{i}\right\}_{I},\left(\bigoplus_{I}\left(g_{0}^{i} \otimes g_{1}^{i}\right)\right) \circ g\right) \\
\sim\left(f,\left\{f_{0}^{i} \circ m_{0}^{i} \circ g_{0}^{i}\right\}_{I},\left\{f_{1}^{i} \circ m_{1}^{i} \circ g_{1}^{i}\right\}_{I}, g\right), \quad f_{j}^{i}, g_{j}^{i} \in F, \\
\left(f,\left\{m_{0}^{i}\right\}_{I},\left\{m_{1}^{i}\right\}_{I}, g\right) \sim\left(f,\left\{m_{0}^{i}\right\}_{I} \cup\left\{\operatorname{id}_{[0]}\right\},\left\{m_{1}^{i}\right\}_{I} \cup\left\{\operatorname{id}_{[0]}\right\}, g\right), \\
\left(f,\left\{a^{i} \otimes m_{0}^{i}\right\}_{I},\left\{m_{1}^{i}\right\}_{I}, g\right) \sim\left(f,\left\{m_{0}^{i}\right\}_{I},\left\{a^{i} \otimes m_{1}^{i}\right\}_{I}, g\right), \quad a^{i} \in A, \\
\left(f,\left\{m_{0}^{i} \oplus m_{0}^{i^{\prime}}\right\}_{I},\left\{m_{1}^{i}\right\}_{I}, g\right) \sim\left(f,\left\{m_{0}^{j}\right\}_{I \oplus I^{\prime}},\left\{m_{1}^{\pi(j)}\right\}_{I \oplus I^{\prime}}, g\right), \\
\left(f,\left\{m_{0}^{i}\right\}_{I},\left\{m_{1}^{i} \oplus m_{1}^{i^{\prime}}\right\}_{I}, g\right) \sim\left(f,\left\{m_{0}^{\pi(j)}\right\}_{I \oplus I^{\prime}},\left\{m_{1}^{j}\right\}_{I \oplus I^{\prime}}, g\right),
\end{gathered}
$$

with $I \stackrel{\sim}{\rightarrow} I^{\prime}, i \mapsto i^{\prime}$, and $\pi: I \oplus I^{\prime} \rightarrow I, \pi(i)=i=\pi\left(i^{\prime}\right)$; here $m_{j}^{i}: I \rightarrow M_{j}, m_{j}^{i^{\prime}}: I^{\prime} \rightarrow M_{j}$ are two sequences of elements of $M_{j}$. One checks that the following operations are well defined, independent of the chosen representatives, and make $M_{0} \otimes_{A} M_{1}$ into an $A$-module satisfying the universal property (8.15.6):

$$
\begin{aligned}
& f \circ\left(g,\left\{m_{0}^{i}\right\}_{I},\left\{m_{1}^{i}\right\}_{I}, g^{\prime}\right) / \sim \circ f^{\prime} \stackrel{\text { def }}{=}\left(f \circ g,\left\{m_{0}^{i}\right\}_{I},\left\{m_{1}^{i}\right\}_{I}, g^{\prime} \circ f^{\prime}\right) / \sim, f, f^{\prime}, g, g^{\prime} \in F \\
& a \otimes\left(f,\left\{m_{0}^{i}\right\}_{I},\left\{m_{1}^{i}\right\}_{I}, g\right) / \sim \stackrel{\text { def }}{=}\left(\operatorname{id}_{W} \otimes f,\left\{a \otimes m_{0}^{i}\right\}_{I},\left\{m_{1}^{i}\right\}_{I}, \mathrm{id}_{Z} \otimes g\right) / \sim, a \in A_{W, Z}, \\
&\left(f,\left\{m_{0}^{i}\right\}_{I},\left\{m_{1}^{i}\right\}_{I}, f^{\prime}\right) / \sim \oplus\left(g,\left\{m_{0}^{j}\right\}_{J},\left\{m_{1}^{j}\right\}_{J}, g^{\prime}\right) / \sim \stackrel{\text { def }}{=}\left(f \oplus g,\left\{m_{0}^{k}\right\}_{k \in I \oplus J},\left\{m_{1}^{k}\right\}_{k \in I \oplus J}, f^{\prime} \oplus g^{\prime}\right) .
\end{aligned}
$$

For $m_{i} \in\left(M_{i}\right)_{Y_{i}, X_{i}}$ we have

$$
m_{0} \otimes m_{A} \stackrel{\text { def }}{=}\left(\operatorname{id}_{Y_{0} \otimes Y_{1}},\left\{m_{0}\right\},\left\{m_{1}\right\}, \operatorname{id}_{X_{0} \otimes X_{1}}\right) / \sim \in\left(M_{0} \otimes_{A} M_{1}\right)_{Y_{0} \otimes Y_{1}, X_{0} \otimes X_{1}}
$$

and hence

$$
\left(f,\left\{m_{0}^{i}\right\}_{I},\left\{m_{1}^{i}\right\}_{I}, g\right) / \sim=f \circ\left(\bigoplus_{i \in I}\left(m_{0}^{i} \otimes_{A} m_{1}^{i}\right)\right) \circ g .
$$

We can similarly define the multilinear functions $\mathcal{B i l i n}_{F}^{A}\left(M_{0}, \ldots, M_{l} ; N\right)$ and the $A$-module $M_{0} \otimes_{A} \cdots \otimes_{A} M_{l}$ representing them.

The construction of the tensor product $M_{0} \otimes_{A} M_{1}$ is functorial in the $M_{i}$ and makes $A$ - $\mathcal{M o d}$ into a symmetric monoidal category with unit element $A$. For $\varphi_{i}: M_{i} \rightarrow N_{i}$ we have

$$
\begin{gathered}
\varphi_{0} \otimes \varphi_{1}: M_{0} \otimes_{A} M_{1} \rightarrow N_{0} \otimes_{A} N_{1}, \\
\varphi_{0} \otimes \varphi_{1}\left(f \circ\left(\bigoplus_{i \in I}\left(m_{0}^{i} \otimes_{A} m_{1}^{i}\right)\right) \circ g\right)=f \circ\left(\bigoplus_{i \in I}\left(\varphi_{0}\left(m_{0}^{i}\right) \otimes \varphi_{1}\left(m_{1}^{i}\right)\right)\right) \circ g,
\end{gathered}
$$




\section{NON-ADDITIVE GEOMETRY}

satisfying

$$
\operatorname{id}_{M_{0}} \otimes \operatorname{id}_{M_{1}}=\operatorname{id}_{M_{0} \otimes M_{1}}
$$

and for $\psi_{i}: N_{i} \rightarrow L_{i}$,

$$
\left(\psi_{0} \otimes \psi_{1}\right) \circ\left(\varphi_{0} \otimes \varphi_{1}\right)=\left(\psi_{0} \circ \varphi_{0}\right) \otimes\left(\psi_{1} \circ \varphi_{1}\right)
$$

and there are canonical isomorphisms

$$
\begin{gathered}
M_{0} \otimes M_{1} \stackrel{\sim}{\longrightarrow} M_{1} \otimes M_{0}, \quad m_{0} \otimes m_{1} \mapsto m_{1} \otimes m_{0}, \\
\left(M_{0} \otimes M_{1}\right) \otimes M_{2} \stackrel{\sim}{\longrightarrow} M_{0} \otimes\left(M_{1} \otimes M_{2}\right) \stackrel{\sim}{\longrightarrow} M_{0} \otimes M_{1} \otimes M_{2}, \\
\left(m_{0} \otimes m_{1}\right) \otimes m_{2} \mapsto m_{0} \otimes\left(m_{1} \otimes m_{2}\right) \mapsto m_{0} \otimes m_{1} \otimes m_{2}, \\
A \otimes_{A} M \stackrel{\sim}{\longrightarrow} M, a \otimes_{A} m \mapsto a \otimes m .
\end{gathered}
$$

Given a homomorphism of $F$-monoids $\varphi: A \rightarrow B$, a $B$-module $N$ can be considered as an $A$ module $N_{A}$ via $a \otimes n:=\varphi(a) \otimes n$, and the functor

$$
B-\mathcal{M o d} \rightarrow A-\mathcal{M o d}, \quad N \mapsto N_{A},
$$

has as left-adjoint the functor

$$
\begin{gathered}
A-\mathcal{M o d} \rightarrow B-\mathcal{M o d}, \quad M \mapsto B \otimes_{A} M, \\
\operatorname{Hom}_{A-\mathcal{M o d}}\left(M, N_{A}\right)=\operatorname{Hom}_{B-\mathcal{M o d}}\left(B \otimes_{A} M, N\right) \\
\varphi \mapsto \varphi\left(g \circ\left(\bigoplus_{I} b_{i} \otimes m_{i}\right) \circ g^{\prime}\right)=g \circ\left(\bigoplus_{I} b_{i} \otimes \varphi\left(m_{i}\right)\right) \circ g^{\prime}, \\
\varphi(m)=\varphi\left(\operatorname{id}_{Y} \circ\left(1_{B} \otimes m\right) \circ \operatorname{id}_{X}\right) \hookleftarrow \varphi .
\end{gathered}
$$

We have canonical isomorphisms

$$
\begin{gathered}
B \otimes_{A}\left(\coprod_{i \in I} M^{i}\right)=\coprod_{i \in I}\left(B \otimes_{A} M^{i}\right), \\
B \otimes_{A}\left(\underset{I}{\lim _{I}} M^{i}\right)=\underset{I}{\lim }\left(B \otimes_{A} M^{i}\right), \quad \text { for a functor } i \mapsto M^{i}, I \rightarrow A-\mathcal{M} \text { od, } \\
B \otimes_{A}(A \cdot \mathcal{S})=B \cdot \mathcal{S}, \quad \text { for a set } \mathcal{S} \text { over }|\mathbb{F}| \times|\mathbb{F}|, \\
\left.B \otimes_{A}\left(M / \varphi\left(M_{0}\right)\right)=\left(B \otimes_{A} M\right) /\left(\operatorname{id}_{B} \otimes \varphi\left(B \otimes_{A} M_{0}\right)\right), \quad \text { for a map of } A \text {-modules } \varphi: M_{0} \rightarrow M .17 .5\right)
\end{gathered}
$$

Given homomorphisms of $F$-monoids $\varphi^{0}: A \rightarrow B^{0}, \varphi^{1}: A \rightarrow B^{1}$, we can view $B^{0}, B^{1}$ as $A$-modules and form their tensor product $B^{0} \otimes_{A} B^{1}$. By the universal property (8.15.6), there is a map

$$
\begin{gathered}
\left(B^{0} \otimes_{A} B^{1}\right) \times\left(B^{0} \otimes B^{1}\right) \rightarrow B^{0} \otimes B^{1}, \\
\left(b^{0} \otimes b^{1}, \bar{b}^{0} \otimes \bar{b}^{1}\right) \mapsto\left(b^{0} \otimes \bar{b}^{0}\right) \otimes\left(b^{1} \otimes \bar{b}^{1}\right)
\end{gathered}
$$

making $B^{0} \otimes_{A} B^{1}$ into an $F$-monoid, and it is the sum of $B^{0}, B^{1}$ over $A$ in $F$-Mon: for every $C \in F-\mathcal{M} o n$,

$$
\begin{aligned}
\operatorname{Hom}_{F-\mathcal{M} o n}\left(B^{0} \otimes_{A} B^{1}, C\right) & =\operatorname{Hom}_{F-\mathcal{M} o n}\left(B^{0}, C\right) \underset{\operatorname{Hom}_{F-\mathcal{M} o n}(A, C)}{\times} \operatorname{Hom}_{F-\mathcal{M} o n}\left(B^{1}, C\right) \\
& =\left\{\left(\psi^{0}, \psi^{1}\right) \mid \psi^{i}: B^{i} \rightarrow C, \psi^{0} \circ \varphi^{0}=\psi^{1} \circ \varphi^{1}\right\} .
\end{aligned}
$$

Here

$$
\psi \mapsto\left(\psi \circ j^{0}, \psi \circ j^{1}\right)
$$




\section{J. Shai HARAN}

with

$$
\begin{gathered}
j^{i}: B^{i} \rightarrow B^{0} \otimes_{A} B^{1}, j^{0}(b)=b \otimes 1_{B^{1}}, j^{1}(b)=1_{B^{0}} \otimes b ; \\
m_{C} \circ\left(\psi^{0} \otimes \psi^{1}\right) \longleftrightarrow\left(\psi^{0}, \psi^{1}\right)
\end{gathered}
$$

with $m_{C}: C \otimes_{A} C \rightarrow C$ the multiplication map of $C, c_{1} \otimes_{A} c_{2} \mapsto c_{1} \otimes c_{2}$.

Example 8.19. For commutative rings $B^{0}, B^{1}$, considering $\mathbb{F}\left(B^{j}\right)$ as $\mathbb{F}$-monoids, we have the tensor product $\mathbb{F}\left(B^{0}\right) \otimes_{\mathbb{F}} \mathbb{F}\left(B^{1}\right)$. It is easy to see that every element $f \circ\left(\bigoplus_{I}\left(b_{i}^{0} \otimes b_{i}^{1}\right)\right) \circ g \in\left(\mathbb{F}\left(B^{0}\right) \otimes_{\mathbb{F}}\right.$ $\left.\mathbb{F}\left(B^{1}\right)\right)_{Y, X}$ is equivalent to an (essentially unique) such element with $b_{i}^{j} \in B^{j} \backslash\{0\}$, and with $f: I \hookrightarrow$ $Y, g^{\mathrm{t}}: I \hookrightarrow X$ embeddings. Thus $\mathbb{F}\left(B^{0}\right) \otimes_{\mathbb{F}} \mathbb{F}\left(B^{1}\right)$ is the $\mathbb{F}$-monoid underlying the $\mathbb{F}$-ring $\mathbb{F}\left\langle B^{0} \times_{0} B^{1}\right\rangle$ associated with $B^{0} \times_{0} B^{1}=B^{0} \times B^{1} /\left(b_{0}, 0\right) \sim\left(0, b_{1}\right) \in \mathcal{M o n}_{0,1}$. On the other hand, if the $B^{j}$ are $A$ algebras, with $A$ a commutative ring (e.g., $A=\mathbb{Z}$ ), we can consider $\mathbb{F}(A), \mathbb{F}\left(B^{j}\right)$ as $\mathbb{F}(A)$-monoids, and we have the tensor product $\mathbb{F}\left(B^{0}\right) \otimes_{\mathbb{F}(A)} \mathbb{F}\left(B^{1}\right)$. Every element $c \in\left(\mathbb{F}\left(B^{0}\right) \otimes_{\mathbb{F}(A)} \mathbb{F}\left(B^{1}\right)\right)_{Y, X}$ is determined by its matrix coefficients

$$
c_{y, x}=j_{y}^{\mathrm{t}} \circ c \circ j_{x} \in\left(\mathbb{F}\left(B^{0}\right) \otimes_{\mathbb{F}(A)} \mathbb{F}\left(B^{1}\right)\right)_{[1],[1]} .
$$

Every element

$$
f \circ\left(\bigoplus_{I}\left(b_{i}^{0} \otimes b_{i}^{1}\right)\right) \circ g \in\left(\mathbb{F}\left(B^{0}\right) \otimes_{\mathbb{F}(A)} \mathbb{F}\left(B^{1}\right)\right)_{[1],[1]}
$$

is equivalent to such an element with $b_{i}^{j} \in B^{j}$, and with $f_{1, i}=g_{i, 1}=1$, which we may denote $\sum_{I} b_{i}^{0} \otimes b_{i}^{1}$. We have well-defined addition of such elements,

$$
\sum_{I} b_{i}^{0} \otimes b_{i}^{1}+\sum_{J} b_{j}^{0} \otimes b_{j}^{1}=\sum_{I \oplus J} b_{k}^{0} \otimes b_{k}^{1}
$$

and we have $A$-action with

$$
a \cdot \sum_{I} b_{i}^{0} \otimes b_{i}^{1}=\sum_{I} a \cdot b_{i}^{0} \otimes b_{i}^{1}=\sum_{I} b_{i}^{0} \otimes a \cdot b_{i}^{1},
$$

and moreover

$$
b_{1}^{0} \otimes b^{1}+b_{2}^{0} \otimes b^{1}=\left(b_{1}^{0}+b_{2}^{0}\right) \otimes b^{1}, b^{0} \otimes b_{1}^{1}+b^{0} \otimes b_{2}^{1}=b^{0} \otimes\left(b_{1}^{1}+b_{2}^{1}\right) .
$$

Thus $\mathbb{F}\left(B^{0}\right) \otimes_{\mathbb{F}(A)} \mathbb{F}\left(B^{1}\right)$ is the $\mathbb{F}(A)$-monoid $\mathbb{F}\left(B^{0} \otimes_{A} B^{1}\right)$, and $B \mapsto \mathbb{F}(B)$ is a tensor functor: Ring $/ A \rightarrow \mathbb{F}(A)-\mathcal{M}$ on.

Varying the base $\mathbb{F}$-ring $F$. We can change the base $\mathbb{F}$-ring. Given a map $\varphi: F_{1} \rightarrow F_{2}$ of $\mathbb{F}$-rings, every $F_{2}$-monoid $B$ is an $F_{1}$-monoid via $\varphi$, giving a functor

$$
F_{2}-\mathcal{M} \text { on } \rightarrow F_{1}-\mathcal{M} \text { on }, \quad B \mapsto B_{F_{1}} .
$$

Conversely, given an $F_{1}$-monoid $A$, its base change $A_{F_{2}} \in F_{2}-\mathcal{M} o d$ is an $F_{2}$-monoid (using equation (3.23.8)), and we have the functor left-adjoint to the preceding one

$$
F_{1}-\mathcal{M} \text { on } \rightarrow F_{2}-\mathcal{M} \text { on }, \quad A \mapsto A_{F_{2}} .
$$

Similarly we can let $F$ vary by working over a fixed $\mathbb{F}$-ringed space $\left(X, \mathcal{O}_{X}\right)$, cf. Definition 6.1 .

Definition 8.20. For $X \in \mathbb{F}$-Rings.Sp, an $\mathcal{O}_{X}$-monoid $A$ is a sheaf of $\mathcal{O}_{X}$-modules such that for $U_{2} \subseteq U_{1} \subseteq X$ open, $A\left(U_{i}\right)$ is an $\mathcal{O}_{X}\left(U_{i}\right)$-monoid, and $A\left(U_{1}\right) \rightarrow A\left(U_{2}\right)$ is an $\mathcal{O}\left(U_{1}\right)$-monoid map, i.e. $A$ is a monoid object of $\mathcal{O}_{X}$-Mod. This gives a category of $\mathcal{O}_{X}$-monoids, $\mathcal{O}_{X}$-Mon, and it has fibred sums. 


\section{NON-ADDITIVE GEOMETRY}

Free monoids. Given an $F$-monoid $A$ and a symbol $\mathfrak{s}: X_{0} \rightarrow Y_{0}, X_{0}, Y_{0} \in|\mathbb{F}|$, take the sequence of symbols $\mathfrak{s}^{n}: X_{0}^{\otimes n} \rightarrow Y_{0}^{\otimes n}, n \geqslant 0$ (where $X_{0}^{\otimes n}=X_{0} \otimes \cdots \otimes X_{0}$ ( $n$ times), and $X_{0}^{\otimes 0}=[1]$ ), and form the free $A$-module on $\left\{\mathfrak{s}^{n}\right\}_{n \geqslant 0}$ :

$$
\begin{aligned}
A[\mathfrak{s}]_{Y, X} & =\left(\coprod_{n \geqslant 0} A \cdot \mathfrak{s}^{n}\right)_{Y, X} \\
& =\left\{f \circ\left(\bigoplus_{0 \leqslant i \leqslant n}\left(a_{i} \otimes s^{i}\right)\right) \circ g \mid a_{i} \in A_{Y_{i}, X_{i}}, f \in F_{Y, \oplus\left(Y_{i} \otimes Y_{0}^{\otimes i}\right)}, g \in F_{\oplus\left(X_{i} \otimes X_{0}^{\otimes i}\right), X}\right\} / \sim
\end{aligned}
$$

with

$$
\left(f \circ \bigoplus\left(f_{i} \otimes \mathrm{id}_{Y_{0}^{\otimes i}}\right)\right) \circ\left(\bigoplus a_{i} \otimes s^{i}\right) \circ\left(\bigoplus\left(g_{i} \otimes \mathrm{id}_{X_{0}^{\otimes i}}\right) \circ g\right) \sim f \circ\left(\bigoplus\left(f_{i} \circ a_{i} \circ g_{i}\right) \otimes s^{i}\right) \circ g .
$$

Using the distributive isomorphism in $\mathbb{F}$ :

$$
\left[\bigoplus_{i}\left(X_{i} \otimes X_{0}^{\otimes i}\right)\right] \otimes\left[\bigoplus_{j}\left(X_{j}^{\prime} \otimes X_{0}^{\otimes j}\right)\right]=\bigoplus_{k}\left[\bigoplus_{i+j=k}\left(X_{i} \otimes X_{j}^{\prime}\right)\right] \otimes X_{0}^{\otimes k},
$$

and similarly with $Y$, we obtain a map

$$
\begin{gathered}
A[\mathfrak{s}] \times A[\mathfrak{s}] \rightarrow A[\mathfrak{s}], \\
\left(f \circ\left(\bigoplus_{i} a_{i} \otimes s^{i}\right) \circ g, f^{\prime} \circ\left(\bigoplus_{j} a_{j}^{\prime} \otimes s^{j}\right) \circ g^{\prime}\right) \mapsto\left(f \otimes f^{\prime}\right) \circ\left(\bigoplus_{k}\left(\bigoplus_{i+j=k} a_{i} \otimes a_{j}^{\prime}\right) \otimes s^{k}\right) \circ\left(g \otimes g^{\prime}\right),
\end{gathered}
$$

which is well defined, $A / F$-bilinear, and gives $A[\mathfrak{s}]$ the structure of $A$-monoid. It is the free $A$-monoid on $\mathfrak{s}$ : for any $A$-monoid $C$,

$$
\operatorname{Hom}_{A-\mathcal{M o n}}(A[\mathfrak{s}], C)=C_{Y_{0}, X_{0}} .
$$

We can similarly form the free $A$-monoid on $\mathfrak{s}_{1}, \ldots, \mathfrak{s}_{l}, \mathfrak{s}_{i}: X_{i} \rightarrow Y_{i}$, and we have

$$
A\left[\mathfrak{s}_{1}, \ldots, \mathfrak{s}_{l}\right]=A\left[\mathfrak{s}_{1}\right] \otimes_{A} \cdots \otimes_{A} A\left[\mathfrak{s}_{l}\right]=A \otimes_{F} F\left[\mathfrak{s}_{1}, \ldots, \mathfrak{s}_{l}\right]=\coprod_{n_{1}, \ldots, n_{l} \geqslant 0} A \cdot \mathfrak{s}^{\left(n_{1}, \ldots, n_{l}\right)}
$$

with $\mathfrak{s}^{\left(n_{1}, \ldots, n_{l}\right)}: X_{1}^{\bigotimes n_{1}} \otimes \cdots \otimes X_{l}^{\bigotimes n_{l}} \rightarrow Y_{1}^{\bigotimes n_{1}} \otimes \cdots \otimes Y_{l}^{\bigotimes n_{l}}$. Taking the direct limit over finite subsets of a set $\mathcal{S}$ over $|\mathbb{F}| \times|\mathbb{F}|$ we have the free $A$-monoid on $\mathcal{S}$

$$
A[\mathcal{S}]=\underset{\left\{\mathfrak{s}_{1}, \ldots, \mathfrak{s}_{l}\right\} \subseteq \mathcal{S}}{\lim } A\left[\mathfrak{s}_{1}, \ldots, \mathfrak{s}_{l}\right]
$$

and $\mathcal{S} \mapsto A[\mathcal{S}]$ is the left-adjoint to the forgetful functor $A$-Mon $\rightarrow \mathcal{S}$ ets $/|\mathbb{F}| \times|\mathbb{F}|$.

Ideals of an F-monoid. An ideal $\mathfrak{a} \subseteq A, A \in F$-Mon, is an $A$-submodule of $A$. Ideals $\mathfrak{a}_{i}$ have intersections $\bigcap_{i} \mathfrak{a}_{i}$, sums $\sum_{i} \mathfrak{a}_{i}$, and finite products $\prod_{i=1}^{n} \mathfrak{a}_{i}$, which are again ideals. The ideal generated by $\mathfrak{s}_{i} \in A_{Y_{i}, X_{i}}$ is given explicitly by

$$
\left(\mathfrak{s}_{1}, \ldots, \mathfrak{s}_{l}\right)=\left\{f \circ\left(\bigoplus_{i} a_{i} \otimes \mathfrak{s}_{i}\right) \circ g \mid f \in F_{Y, \oplus\left(W_{i} \otimes Y_{i}\right)}, g \in F_{\oplus\left(Z_{i} \otimes X_{i}\right), X}, a_{i} \in A_{W_{i}, Z_{i}}\right\} .
$$

Homogeneous ideals $\mathfrak{a}$, i.e. $\mathfrak{a}$ is generated by $\mathfrak{a}_{[1],[1]}$, correspond one-to-one with $H$-ideals, i.e. $\mathfrak{a} \subseteq A_{[1],[1]}$ such that for

$$
\mathfrak{s}_{1}, \ldots, \mathfrak{s}_{l} \in \mathfrak{a}, a_{i} \in A_{W_{i}, Z_{i}}, f \in F_{[1], \oplus W_{i}}, g \in F_{\oplus Z_{i},[1]} \Rightarrow f \circ\left(\bigoplus a_{i} \otimes \mathfrak{s}_{i}\right) \circ g \in \mathfrak{a} .
$$




\section{J. SHAi HARAN}

An ideal $\mathfrak{p} \subseteq A$ (respectively $H$-ideal $\left.\mathfrak{p} \subseteq A_{[1],[1]}\right)$ is called $S$-prime (respectively prime) if $A \backslash \mathfrak{p}$ (respectively $A_{[1],[1]} \backslash \mathfrak{p}$ ) is closed under $\otimes$. We let $\operatorname{SPEC}(A)$ (respectively $\operatorname{Spec}(A)$ ) denote $S$-primes (respectively primes).

Primes and spectra. An ideal $\mathfrak{a} \subseteq A$ (respectively $H$-ideal $\mathfrak{a} \subseteq A_{[1],[1]}$ ) is called $E$-ideal (respectively $H$-E-ideal) if $\mathfrak{a}=Z E(\mathfrak{a})$, respectively $\mathfrak{a}=(Z E(\mathfrak{a}))_{[1],[1]}$, or explicitly

$$
f \circ\left(a_{0} \oplus \bigoplus_{i} a_{i} \otimes \mathfrak{s}_{i}\right) \circ g \in \mathfrak{a} \quad \Leftrightarrow \quad f \circ\left(a_{0} \oplus 0\right) \circ g \in \mathfrak{a}, \quad \text { for } \mathfrak{s}_{i} \in \mathfrak{a}, a_{i} \in A .
$$

We let $E$-SPEC $(A)$ (respectively $E$-Spec $(A))$ denote the $S$ - $E$-primes (respectively $E$-primes). We have the sets

$$
\begin{gathered}
V_{A}(\mathfrak{a})=\{\mathfrak{p} \in \operatorname{SPEC}(A) \mid \mathfrak{p} \supseteq \mathfrak{a}\}, \quad \mathfrak{a} \subseteq A \text { ideal; } \\
D_{A}(f)=\{\mathfrak{p} \in \operatorname{SPEC}(A) \mid \mathfrak{p} \not \supset f\}, \quad f \in A ; \\
V_{A}(\mathfrak{a})=\{\mathfrak{p} \in \operatorname{Spec}(A) \mid \mathfrak{p} \supseteq \mathfrak{a}\}, \mathfrak{a} \subseteq A_{[1],[1]} H \text {-ideal; } \\
D_{A}(f)=\{\mathfrak{p} \in \operatorname{Spec}(A) \mid \mathfrak{p} \not \supset f\}, \quad f \in A_{[1],[1] .}
\end{gathered}
$$

The $V_{A}(\mathfrak{a})$ define the closed sets, the $D_{A}(f)$ define a basis for the open sets of the Zariski topology on $\operatorname{SPEC}(A)$ and on $\operatorname{Spec}(A)$.

For an ideal $\mathfrak{a}$ (respectively $E$-ideal, $H$-ideal, $H$ - $E$-ideal), and for $f \in A$ (respectively $f \in A_{[1],[1]}$ for $H$-ideals) with $f^{\otimes n} \notin \mathfrak{a}$ for all $n$, a maximal element of the set of ideals (respectively $E$-ideals, $H$-ideals, $H$-E-ideals) containing $\mathfrak{a}$ and not containing any $f^{\otimes n}$ is $S$-prime (respectively $S$ - $E$-prime, prime, E-prime), and it follows that

$$
\sqrt{\mathfrak{a}}=\left\{f \in A\left(\text { respectively } A_{[1],[1]}\right) \mid f^{\otimes n} \in \mathfrak{a} \text { for some } n \geqslant 1\right\}=\bigcap_{\mathfrak{a} \subseteq \mathfrak{p}} \mathfrak{p},
$$

where the intersection is taken over all $S$-primes (respectively $S$ - $E$-primes, primes, $E$-primes) containing a. For a subset $X \subseteq \operatorname{SPEC}(A)$ (respectively $E$-SPEC $(A), \operatorname{Spec}(A), E$-Spec $(A)$ ) we have the ideal (respectively $E$-ideal, $H$-ideal, $H$-E-ideal),

$$
I(X)=\bigcap_{\mathfrak{p} \in X} \mathfrak{p},
$$

and

$$
\sqrt{\mathfrak{a}}=I\left(V_{A}(\mathfrak{a})\right), \quad V_{A}(I(X))=\bar{X} .
$$

It follows that we have bijections between the closed sets in $\operatorname{SPEC}(A)$ (respectively $E-\operatorname{SPEC}(A)$, $\operatorname{Spec}(A), E$-Spec $(A)$ ), and the radical $\mathfrak{a}=\sqrt{\mathfrak{a}}$ ideals (respectively $E$-ideals, $H$-ideals, $H$ - $E$-ideals); the irreducible closed sets correspond to $S$-primes (respectively $S$ - $E$-primes, primes, $E$-primes). There is a commutative diagram of spaces

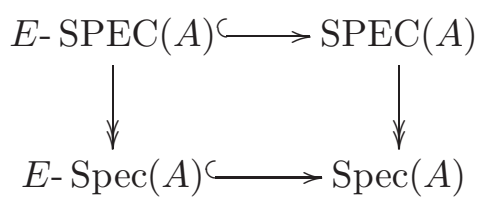

with the horizontal arrows embedding of subspaces (with the subspace topology), and the vertical arrows are surjective continuous maps (to show surjectivity one needs localization, cf. below). For a map of $F$-monoids $\varphi: A \rightarrow B$, the pull-back along $\varphi, \varphi^{*}(\mathfrak{p})=\varphi^{-1}(\mathfrak{p})$, gives a map from the diagram (8.22.8) associated with $B$ to the one associated with $A$. Since

$$
\varphi^{*-1} V_{A}(\mathfrak{a})=V_{B}(\varphi(\mathfrak{a})), \quad \varphi^{*-1} D_{A}(f)=D_{B}(\varphi(f)),
$$




\section{NON-ADDITIVE GEOMETRY}

$\varphi^{*}$ is continuous, and we have four functors from $F$-Mon to the category of compact sober spaces and continuous maps.

Localization and structure sheaf $\mathcal{O}_{A}$. The theory of localization of an $F$-monoid $A$ (respectively $A$-module $M)$ with respect to a multiplicative subset $S \subseteq A_{[1],[1]}$ goes exactly as in $\S 5$, and produces an $F$-monoid $S^{-1} A$ (respectively $S^{-1} A$-module $S^{-1} M$ ). The functor $A \mapsto S^{-1} A$ (respectively $\left.M \mapsto S^{-1} M\right)$ commutes with direct limits, tensor products, finite inverse limits, free objects; in particular it preserves kernels $\varphi^{-1}(0), \mathcal{K} \mathcal{E} \mathcal{R}(\varphi)$, cokernels, and commutes with the operations $E, Z$. Propositions 5.12, 5.13, and 5.14 remain valid: surjectivity or injectivity of a map in $A-\mathcal{M o d}$ can be checked locally at every prime (or maximal $H$-ideal). For an $F$-monoid $A$ (respectively $A$-module $M)$, and for $\mathfrak{p} \in \operatorname{Spec}(A)$, or for $f \in A_{[1],[1]}$, we put

$$
\begin{gathered}
A_{\mathfrak{p}}=S_{\mathfrak{p}}^{-1} A\left(\text { respectively } M_{\mathfrak{p}}=S_{\mathfrak{p}}^{-1} M\right), \quad S_{\mathfrak{p}}=A_{[1],[1]} \backslash \mathfrak{p} \\
A_{f}=S_{f}^{-1} A\left(\text { respectively } M_{f}=S_{f}^{-1} M\right), \quad S_{f}=\left\{f^{n} \mid n \geqslant 0\right\} .
\end{gathered}
$$

Over $\operatorname{Spec}(A)$ we have a sheaf $\mathcal{O}_{A}$ of $F$-monoids (respectively a sheaf of $\mathcal{O}_{A}$-modules $\widetilde{M}$ ) such that

$$
\mathcal{O}_{A}\left(D_{A}(f)\right)=A_{f}\left(\text { respectively } \widetilde{M}\left(D_{A}(f)\right)=M_{f}\right), \quad f \in A_{[1],[1]},
$$

and with stalks at $\mathfrak{p} \in \operatorname{Spec}(A)$ given by

$$
\mathcal{O}_{A, \mathfrak{p}}=A_{\mathfrak{p}}\left(\text { respectively }\left.\widetilde{M}\right|_{\mathfrak{p}}=M_{\mathfrak{p}}\right) .
$$

The proof of (8.23.2) goes exactly as for $\mathbb{F}$-rings, cf. Proposition 5.19. The proof of (8.23.1) goes as for $\mathbb{F}$-rings, cf. Proposition 5.20, with only a minor change at the end: since the sets $D_{A}\left(g_{i}\right)$ cover $D_{A}(f)$ we have

$$
f^{m}=f_{1} \circ\left(\bigoplus_{i} g_{i} \otimes b_{i}\right) \circ f_{2}, \quad \text { with } f_{i} \in F, b_{i} \in A,
$$

and we let $a=\left(\operatorname{id}_{Y} \otimes f_{1}\right) \circ\left(\bigoplus_{i} a_{i} \otimes b_{i}\right) \circ\left(\operatorname{id}_{X} \otimes f_{2}\right)$, giving again $g_{j} \otimes a=f^{m} \otimes a_{j}$.

Stalk $\mathcal{O}_{A, \mathfrak{p}}$ is a local $F$-monoid, having a unique maximal $H$-ideal $\mathfrak{m}_{\mathfrak{p}}$, and $F_{\mathfrak{p}}=\mathcal{O}_{A, \mathfrak{p}} / \mathfrak{m}_{\mathfrak{p}}$ is a field in F-Mon. We remark that we have again four notions of fields (and four notions of local $F$-monoids) given by the conditions in Definition 4.23.

A map of $F$-monoid $\varphi: A \rightarrow B$ induces the localized map

$$
\varphi^{\sharp}: \mathcal{O}_{A}(U) \rightarrow \mathcal{O}_{B}\left(\varphi^{*-1} U\right), \quad U \subseteq \operatorname{Spec}(A),
$$

giving rise to a local homomorphism

$$
\varphi_{\mathfrak{q}}^{\sharp}: A_{\varphi^{*}(\mathfrak{q})} \rightarrow B_{\mathfrak{q}}, \quad \varphi_{\mathfrak{q}}^{\sharp}\left(\mathfrak{m}_{\varphi *(\mathfrak{q})}\right) \subseteq \mathfrak{m}_{\mathfrak{q}}, \quad \mathfrak{q} \in \operatorname{Spec}(B),
$$

and a commutative diagram with $\mathfrak{p}=\varphi^{*}(\mathfrak{q}), \mathfrak{q} \in \operatorname{Spec}(B)$ as follows:

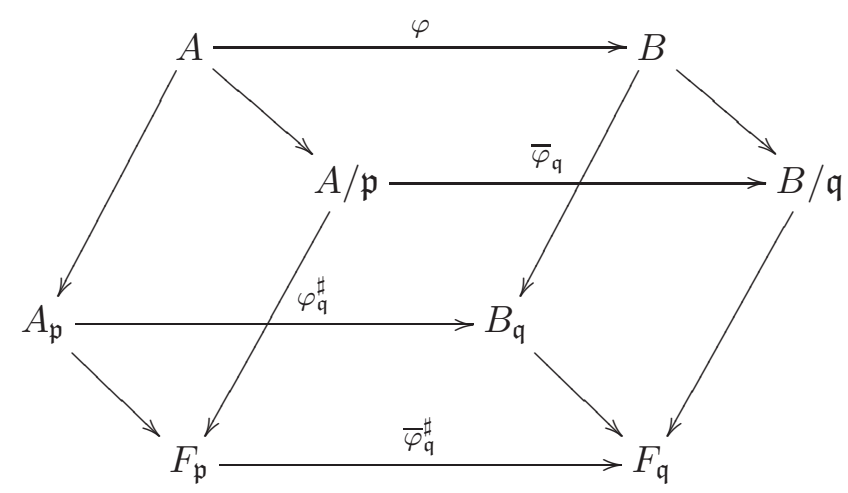




\section{NON-ADDITIVE GEOMETRY}

One defines the categories of $F$-monoid spaces, F-Mon.S $p$ (respectively of local $F$-monoid spaces, $\mathcal{L}$ oc.F-Mon.Sp) as the category with objects $\left(X, \mathcal{O}_{X}\right), X$ a topological space, $\mathcal{O}_{X}$ a sheaf of $F$-monoids on $X$ (respectively with local $F$-monoids for stalks $\mathcal{O}_{X, x}, x \in X$ ), and with maps $\left(f, f^{\sharp}\right):\left(X, \mathcal{O}_{X}\right) \rightarrow\left(Y, \mathcal{O}_{Y}\right)$, continuous maps $f: X \rightarrow Y$, and maps of sheaves of $F$-monoids over $Y, f^{\sharp}: \mathcal{O}_{Y} \rightarrow f_{*} \mathcal{O}_{X}$ (respectively such that the induced map on stalks $f_{x}^{\sharp}: \mathcal{O}_{Y, f(x)} \rightarrow \mathcal{O}_{X, x}$ is a local homomorphism). Then $A \mapsto\left(\operatorname{Spec}(A), \mathcal{O}_{A}\right)$ is a contravariant functor

$$
\text { Spec : F-Mon } \rightarrow \text { Loc.F-Mon.Sp }
$$

which is the adjoint to the global section functor

$$
\Gamma: \mathcal{L} \text { oc.F-Mon.Sp } \rightarrow \text { F-Mon }, \quad \Gamma\left(X, \mathcal{O}_{X}\right)=\mathcal{O}_{X}(X),
$$

so that we have

$$
\operatorname{Hom}_{\mathcal{L} o c . F-\mathcal{M} o n . \mathcal{S} p}(X, \operatorname{Spec} A)=\operatorname{Hom}_{F-\mathcal{M} o n}\left(A, \Gamma\left(X, \mathcal{O}_{X}\right)\right) .
$$

Definition 8.24. A Zariski $F$-monoid scheme is a local $F$-monoid space $\left(X, \mathcal{O}_{X}\right)$ such that there exists an open covering $X=\bigcup_{i} U_{i}$, with $\left(U_{i},\left.\mathcal{O}_{X}\right|_{U_{i}}\right) \simeq \operatorname{Spec} \mathcal{O}_{X}\left(U_{i}\right)$. Maps of Zariski $F$-monoid schemes are maps of $\mathcal{L}$ oc.F-Mon.Sp, thus we have a full subcategory of $\mathcal{L}$ oc.F-Mon.Sp consisting of Zariski $F$-monoid schemes.

\section{REFERENCES}

Dei05 A. Deitmar, Schemes over $\mathbb{F}_{1}$, in Number fields and Function fields - two parallel worlds, Progress in Mathematics, vol. 239 (Birkhäuser, Boston, 2005).

Fal92 G. Faltings, Lectures on the arithmetic Riemann-Roch theorem, Ann. of Math. Stud., vol. 127 (Princeton University Press, Princeton, NJ, 1992).

Gro60 A. Grothendieck, Elements de Geometrie Algébrique, I. Le Langage des Schémas, Publ. Math. Inst. Hautes Études Sci. 4 (1960).

Har90 S. M. J. Haran, Index theory, potential theory, and the Riemann hypothesis, L-functions and arithmetic, Durham 1990, London Math. Soc. Lecture Series, vol. 153 (Cambridge University Press, 1990).

Har01 S. M. J. Haran, The mysteries of the real prime (Oxford University Press, 2001).

Har06 S. M. J. Haran, Arithmetical investigations: representation theory, orthogonal polynomials, and quantum interpolation, COE Lecture Notes (Kyushu Univ. Press, Fukuoka, 2006).

Hart77 R. Hartshorne, Algebraic geometry (Springer, 1977).

KOW03 N. Kurokawa, H. Ochiai and M. Wakayama, Absolute derivations and zeta functions, Doc. Math. (2003), extra vol. 565-584.

Man95 Y. I. Manin, Lectures on zeta functions and motives (according to Deninger and Kurokawa), Columbia University Number Theory Seminar, New York, 1992, Asterisque, no. 228, 4 (1995), $121-163$.

Qui73 D. Quillen, Higher algebraic K-theory, Lecture Notes in Mathematics, vol. 341 (Springer, 1973), $85-147$.

Sou04 C. Soulé, Les variétés sur le corps à un élément, Moscow Math. J. 4 (2004), 217-244.

SABK92 C. Soulé, D. Abramovich, J. F. Burnol and J. Kramer, Lectures on Arakelov geometry, Studies in Advanced Mathematics, vol. 33 (Cambridge University Press, 1992).

TV05 B. Toën and M. Vaquié, Au-dessous de Spec $\mathbb{Z}$, Preprint (2005), arXiv:math.AG/0509684v1.

M. J. Shai Haran haran@tx.technion.ac.il

Department of Mathematics, Technion - Israel Institute of Technology, 32000 Haifa, Israel 